\title{
Mapping chromatin accessibility and active regulatory elements reveals new pathological mechanisms in human gliomas
}

\author{
Authors/Affiliations
}

\author{
Karolina Stępniak ${ }^{1 *}$, Magdalena A. Machnicka ${ }^{2 *}$, Jakub Mieczkowski ${ }^{1 *}$, Anna \\ Macioszek², Bartosz Wojtaś', Bartłomiej Gielniewski', Sylwia K. Król'1, Rafał Guzik', \\ Michał J. Dąbrowski ${ }^{3}$, Michał Dramiński ${ }^{3}$, Marta Jardanowska ${ }^{3}$, llona Grabowicz ${ }^{3}$, Agata \\ Dziedzic $^{3}$, Hanna Kranas ${ }^{2 a}$, Karolina Sienkiewicz ${ }^{2}$, Klev Diamanti ${ }^{4}$, Katarzyna Kotulska ${ }^{5}$, \\ Wiesława Grajkowska ${ }^{5}$, Marcin Roszkowski ${ }^{5}$, Tomasz Czernicki ${ }^{6}$, Andrzej Marchel ${ }^{6}$, \\ Jan Komorowski ${ }^{4}$, Bozena Kaminska \#, Bartek Wilczyński \# \\ ${ }^{1}$ Nencki Institute of Experimental Biology of the Polish Academy of Sciences; Warsaw, \\ 02-093, Poland \\ ${ }^{2}$ Faculty of Mathematics, Informatics and Mechanics, University of Warsaw, Warsaw, \\ 02-097, Poland \\ ${ }^{3}$ Institute of Computer Science of the Polish Academy of Sciences; Warsaw, 01-248 Poland \\ ${ }^{4}$ Department of Cell and Molecular Biology, Uppsala University, Uppsala, 75124, Sweden \\ ${ }^{5}$ Deptartments of Neurology, Neurosurgery, Neuropathology, The Children's Memorial \\ Health Institute, Warsaw, 02-093, Poland \\ ${ }^{6}$ Department of Neurosurgery, Medical University of Warsaw, Warsaw, 02-097, Poland.
}

\section{${ }^{*}$ Equally contributing authors \\ \# Corresponding authors}

Contact Info: b.wilczynski@mimuw.edu.pl; b.kaminska@nencki.gov.pl

\section{Summary}

Chromatin structure and accessibility, and combinatorial binding of transcription factors to regulatory elements in genomic DNA control transcription. Genetic variations in genes encoding histones, epigenetics-related enzymes or modifiers affect chromatin structure/dynamics and result in alterations in gene expression contributing to cancer development or progression. Gliomas are brain tumors frequently associated with epigenetics-related gene deregulation. We performed whole-genome mapping of chromatin accessibility, histone modifications, DNA methylation patterns and transcriptome analysis simultaneously in multiple tumor samples to unravel novel epigenetic dysfunctions driving gliomagenesis. Based on the results of the integrative analysis of the acquired profiles, we created an atlas of active enhancers and promoters in benign and malignant gliomas. We explored these elements and intersected with $\mathrm{Hi}-\mathrm{C}$ data to uncover molecular

a Current address: Institute for Research in Biomedicine (IRB Barcelona), The Barcelona Institute of Science and Technology, Baldiri Reixac, 10, 08028 Barcelona, Spain 
mechanisms instructing gene expression in gliomas.

\section{Keywords}

Gliomas; chromatin accessibility; histone modifications; ATAC-sequencing; RNA sequencing; topological domains; gliomagenesis

\section{Significance}

Epigenetics-driven deregulation of gene expression accompanies cancer development, but its comprehensive characterization in cancer patients is fragmentary. We performed wholegenome profiling of gene expression, open chromatin, histone modifications and DNAmethylation profiles in the same samples from benign and malignant gliomas. Our study provides a first comprehensive atlas of active regulatory elements in gliomas, which allowed identification of the functional enhancers and promoters in patient samples. This comprehensive approach revealed epigenetic patterns influencing gene expression in benign gliomas and a new pathogenic mechanism involving FOXM1-driven network in glioblastomas. This atlas provides a common set of elements for cross-comparisons of existing and new datasets, prompting novel discoveries and better understanding of gliomagenesis.

\section{Highlights}

- We provide an atlas of cis-regulatory elements active in human gliomas

- Enhancer-promoter contacts operating in gliomas are revealed

- Diverse enhancer activation is pronounced in malignant gliomas

- Chromatin loop activates FOXM1-ANXA2R pathological network in glioblastomas.

\section{INTRODUCTION}

Gliomas are most common primary tumors of the central nervous system (CNS). According to a World Health Organization (WHO) classification, gliomas are divided based on molecular dysfunctions and histopathology into WHO grade I gliomas (benign tumors with infrequent genomic alterations and longer-term survival), grades II and III diffuse gliomas (characterized by the presence or absence of mutations in IDH, TERT, ATRX and copy number alterations of $1 p, 19 q, 7$, and $10 q$ ) and grade IV glioblastoma, the most malignant tumor with numerous genomic alterations (Ceccarelli et al., 2016; Louis et al., 2016). Glioma development is a multistep process, involving genetic and epigenetic alterations (Reifenberger et al., 2016). Comprehensive sequencing studies have revealed numerous aberrations in epigenetics- and chromatin remodeling-related genes in high grade gliomas. Due to aberrant activation or inactivation of epigenetics-related enzymes the epigenetic landscape is highly dysregulated leading to aberrant gene expression (Fontebasso et al., 2013; Maleszewska and Kaminska, 2013).

Chromatin acts as a dynamic platform of signal integration and a long-term maintenance of gene expression profiles. Modifications of histones, particularly their $\mathrm{N}$-terminal tails, 
influence chromatin accessibility and gene expression, and can be activating or repressive (Kouzarides, 2007). Acetylation of $\mathrm{N}$-terminal lysine residues of histones $\mathrm{H} 3$ and $\mathrm{H} 4$, trimethylation of $\mathrm{H} 3$ lysine $4(\mathrm{H} 3 \mathrm{~K} 4 \mathrm{me} 3)$ and lysine $79(\mathrm{H} 3 \mathrm{~K} 79 \mathrm{me})$, and trimethylation of $\mathrm{H} 3$ lysine $36(\mathrm{H} 3 \mathrm{~K} 36 \mathrm{~m} 3)$, are typically associated with an active chromatin, while methylation of lysines 9 (H3K9me3) and 27 (H3K27me3) of histone H3 are hallmarks of a condensed chromatin at silent loci (Tessarz and Kouzarides, 2014). H3 acetylation and H3K4me1 marks outside of promoter regions have been correlated with functional enhancers in different cell types (Bogdanovic et al., 2012; Bonn et al., 2012). The deceptively opposite modifications, H3K4me3 and H3K27me3 co-localize in regions termed "bivalent domains", mostly in embryonic stem cells (Bernstein et al., 2006). Extensive characterization of histone methylations has been completed at a limited genome coverage and resolution in selected human cells (Bernstein et al., 2007). There are only a few reports regarding profiles of histone modifications or chromatin accessibility in freshly resected human specimens. This includes high-resolution $\mathrm{Hi}-\mathrm{C}$ maps of chromatin contacts in human embryonic brain (Won et al., 2016), ATAC-seq on postmortem brain (Fullard et al., 2017) or glioma stem like cells from glioblastoma (Tome-Garcia et al., 2018), ChIP-seq analysis for H3K27me3 modification on 11 glioblastoma and 2 diffuse astrocytoma samples (Sharma et al., 2017). Only recently researchers began integrating H3K27ac profiles with gene expression, DNA methylomes, copy number variations, and whole exomes demonstrating distinct chromatin and epigenetic profiles in glioblastomas, distinguishing this entity from other brain tumors (Mack et al., 2019). Combination of those features in a single patient and intersections of acquired profiles in gliomas of different grades have not yet been achieved.

We performed a comprehensive analysis of whole genome profiles of open chromatin, histone modifications, DNA methylation and gene expression in a given patient using freshly resected gliomas of various malignancy grades. Intersecting the acquired data led to more precise mapping of active promoters and enhancers in gliomas, some reflecting brainspecific sites. We intersected our data with $\mathrm{Hi}-\mathrm{C}$ maps from a human embryonic brain to identify topologically associated domains and we searched for co-regulated genes, regulatory binding motifs and potential master regulators. This approach revealed a new regulatory connection between FOXM1 and ANXA2R implicated in gliomagenesis. We provide an atlas of brain specific enhancers and promoters which could be a valuable resource for further exploration. The data are available at http://regulomics.mimuw.edu.pl/GliomaAtlas/ in raw and processed forms ready to visualize in genome browsers.

\section{Results}

\section{Cohort description and experimental workflow}

We focused on groups of patients representing major malignancy groups: pilocytic astrocytomas (WHO grade I, PA, $\mathrm{n}=11$ ), diffuse astrocytomas (WHO grade II and III, DA, $\mathrm{n}=7$ ) and glioblastomas (WHO grade IV, GBM, $n=15$ ). Clinical data about each patient have 
been collected and are presented in Table S1. Freshly resected glioma tissues were immediately homogenized to a single cell suspension and experimentally defined numbers of cells were split into portions that were directly processed for ATAC-seq, ChIP-seq, RNAseq or DNA methylation experiments (Figure 1). Tissue homogenization was performed to avoid confounding factors related to tumor heterogeneity. We analyzed the profiles of DNA methylation, open chromatin and histone modifications characteristic for active (H3K4me3, $\mathrm{H} 3 \mathrm{~K} 27 \mathrm{ac}$ ) and repressed chromatin (H3K27me3) along with RNA-sequencing on the individual patients tissues. The IDH1/2 mutational status has been evaluated in all samples by Sanger sequencing of PCR amplicons (Table S1). All experiments and sequencing were carried out in the same laboratory, therefore, we could avoid possible sequencing artifacts. All datasets were subjected to rigorous quality control according to Encyclopedia of DNA elements (ENCODE) best practices, and only datasets passing stringent quality control metrics are presented.

\section{Creating the atlas of regulatory sites in benign and malignant gliomas}

For each individual, we performed transposase-accessible chromatin with sequencing (ATAC-seq) and chromatin immunoprecipitation followed by sequencing (ChIP-seq) to detect histone modification marks characteristic for active and poised enhancers, and active promoters. Figure 2A summarizes numbers of samples for which ATAC-seq and ChIP-seq were collected excluding the samples that did not meet our stringent quality criteria. For a substantial number of glioma samples we collected complete datasets, except for a lack of H3K27me3 profiles from PAs, due to a low size of the resected tumors. Representative ATAC-seq, ChIP-seq and RNA-seq peaks for the NRXN2 gene in two samples, one selected from pilocytic astrocytomas (PA04) and one from glioblastomas (GB01) are shown in Figure 2B. The peak enrichments are comparable across samples with expected overrepresentation of peaks in promoter regions. Numbers of detected peaks are presented in Figure 2C.

By performing ChIP-seq experiments for two active and one repressive histone marks (H3K4me3, H3K27ac and H3K27me3), ATAC-seq and RNA-seq we have collected a comprehensive dataset describing chromatin state and gene activity across different glioma grades. We identified regulatory regions whose activity is shared between samples ("common") or specific to a small subset of samples ("variable") for both H3K4me3 and H3K27ac ChIP-seq experiments (Figure 2D). We identify many more common than variable H3K4me3 peaks, suggesting those commonly active promoters represent genes expressed in the brain, while the variable ones may be more related to gliomagenesis.

Genome-wide chromatin conformation capture $(\mathrm{Hi}-\mathrm{C})$ allows identification of interactions between DNA regions including promoter-enhancer interactions. To better describe enhancers, we used $\mathrm{Hi}-\mathrm{C}$ data from high-resolution 3D maps of chromatin contacts from developing human brain (Won et al., 2016) for identification of enhancer-promoter contacts that might function in gliomas. First, using $\mathrm{Hi}-\mathrm{C}$ data we searched for contacts of all putative active enhancers identified in our samples with any region within $2 \mathrm{Mbp}$ range 
(Figure 2E). We found significant $(p<0.001)$ contacts for 21,141 putative enhancers out of 45,548 (including 5,530 common enhancers out of 10,673). Target genomic intervals which were predicted to be in contact with common putative enhancers are enriched in promoter regions, supporting biological relevance of these predictions (Suppl. Figure 2A).

\section{Identified, non-promoter regulatory regions show high diversity between patients}

Active promoters, defined by the presence of either H3K4me3 or H3K27ac, are highly consistent between patients. Similarly, gene expression profiles show significant overlap between patients (Figure 3A, Figure S3). Expectedly, sets of promoters identified as active based on the presence of either one of the active histone marks or open chromatin peaks, are highly similar; 8007 common active promoters display ATAC-seq signals, H3K4me3 and H3K27ac marks (Figure 3B). Active promoters exhibit also evolutionary conservation in the context of 100 vertebrate genomes, compared to random genomic intervals (Figure $3 \mathrm{C}$ ). In contrast to promoter regions, putative enhancers show high variability between patients (Figure $3 \mathrm{~A}$, Figure S3). Their sequence conservation is also weaker than sequence conservation of the promoters, but still very significant when compared to random genomic intervals (Figure $3 \mathrm{C}$ ). By comparing these common and variable promoter regions to the published studies of promoters active in normal brain (Bernstein et al. 2012) and in non-brain cell lines, we can estimate that approximately $50 \%$ of common promoters are also active in normal brain and $30 \%$ are also active in other tissues (Figure S3B). The values for variable elements are considerably lower, with approximately $90 \%$ of them not appearing in brain or cell-type derived datasets, making them likely to be specific to gliomas.

Having paired ChIP-seq and RNA-seq data from the same samples we investigated whether the expected correlation between the level of active histone marks and gene expression levels (Karlic et al., 2010) is visible in our data. Both H3K4me3 and H3K27ac ChIP-seq coverages at promoter regions correlate with the expression of the associated transcripts (Spearman $\mathrm{R}=0.62$ and 0.66 , respectively; Figure 3D). However, our data demonstrate that dependency between occurrence of H3K4me3 peaks and transcript expression is quite non-linear, with a pronounced difference in coverage between transcripts whose expression is within 1st or 2nd quantile. In contrast, in case of H3K27ac the increase in the transcript expression is associated with the steady increase in the ChIP-seq signal (Figure 3D).

Hierarchical clustering on the mRNA expression profiles of the samples revealed that malignancy groups correspond very well to the obtained clusters (Figure 3E). All but one PA samples formed a distinct cluster showing a stronger within-group similarity than groups related to other malignancies. As expected due to the presence of PA-like glioma subtype within LGr4 RNA cluster (Ceccarelli et al. 2016), majority of the PA samples were classified to LGr4 Pan Glioma RNA Expression Cluster. DA samples also exhibited a high similarity of 
RNA expression profiles. Majority of them were assigned to LGr1/LGr3 RNA clusters. In concordance with Pan Glioma RNA Expression Cluster specificity, all IDH1 mutant samples were assigned to the LGr1 or LGr3 RNA clusters, even though they were split on the dendrogram into DA and GB specific clusters. The GB mRNA expression profiles exhibited the largest variability, although the majority of them were found to be of Mesenchymal glioblastoma subtype assigned to LGr4 RNA cluster.

To further assess biological relevance of enhancer-promoter contacts predicted in glioma samples, we used $\mathrm{Hi}-\mathrm{C}$ data from a fetal human brain (Won et al., 2016). We computed the Spearman correlation of H3K27ac coverage on enhancers with transcript expression for enhancer-promoter pairs defined either based on $\mathrm{Hi}-\mathrm{C}$ data or by assigning the closest transcript to each analyzed enhancer. We found that both for closest enhancertranscript pairs and pairs identified from $\mathrm{Hi}-\mathrm{C}$ data the correlation between the histone mark level and transcript expression is relatively low (around 0.1 to 0.25 ) (Figure 3F). Our results suggest that closest enhancer-promoter pairs are expected to be correlated if the distance between them is at most $20 \mathrm{~kb}$. However, in cases when no transcript can be found within $200 \mathrm{kbp}$ from the putative enhancer, Hi-C data can provide significantly more information about expected enhancer-promoter interactions than assignment based on the shortest promoter-enhancer distance.

Altogether, the presented results on integrative analysis of ATAC-seq, ChIP-seq and RNA-seq allowed us to identify non-coding, regulatory sites that are active in gliomas and represent a common brain specific sites. We predicted the presence of active promoters and putative enhancers, and predicted enhancer-promoter contacts in our glioma samples by exploiting the published $\mathrm{Hi}-\mathrm{C}$ data dataset (Won et al., 2016). The data allowed us to create an atlas of non-coding, regulatory sites in those brain tumors and we made the data easily available in raw and processed formats in a genome browser on our website (http://regulomics.mimuw.edu.pl/GliomaAtlas)

\section{H3K27ac at the transcription start sites marks genes related to neuronal signaling in pilocytic astrocytomas}

We explored the atlas of active promoters and putative enhancers to identify tumor-specific gene regulation. We compared chromatin activity around transcription start sites (TSS) between benign (PA) and malignant (DA/GB) tumors using $\mathrm{H} 3 \mathrm{~K} 4 \mathrm{me} 3$ and $\mathrm{H} 3 \mathrm{~K} 27$ ac profiles (Figure $4 A B$ ). The analysis was limited to protein coding genes. We found that the abundance of the selected histone marks discriminated the two glioma groups. While H3K4me3 marks at TSS were significantly higher in PA samples (Figure 4A), H3K27ac signals were significantly more abundant in DA/GB samples (Figure 4B, Figure S4A).

Interestingly, besides the global increase of H3K27ac marks in DA/GB, we found a set of regions with higher $\mathrm{H} 3 \mathrm{~K} 27 \mathrm{ac}$ abundance in PA than in DA/GB samples (Figure 4C, we 
will refer to them as the selected regions in the following paragraph). The detailed H3K27ac profiles centered at the corresponding TSS confirmed that the observed increase in their abundance was not an artifact resulting from data transformation, and was spread over the selected region (TSS+/-2kb) (Figure 4D). Also, ATAC-seq profiles obtained for four PA and four DA/GB samples showed differences in chromatin accessibility (Figure 4E). To explore those changes in the genome-wide context, we compared the H3K27ac profiles in the selected regions with the remaining regions. We found that in comparison to the other regions, H3K27ac profiles in the selected regions were substantially lower in DA/GB samples (Figure 4F, right panel), while in PA samples, the H3K27ac levels remained unchanged (Figure 4F, left panel). This shows that H3K27ac mark in the selected regions was diminished in samples of the malignant tumors, which may result from the absence of some regulatory modes or cell types in DA/GB tumors.

To further characterize PA-specific H3K27ac profiles in terms of gene functionality, we analyzed expression levels of the genes corresponding to the selected TSS proximal regions. The average expression levels of these genes were significantly higher $(\mathrm{P}=2.8 \mathrm{e}-$ 167, Wilcoxon rank-sum test) in PA tumors, which supported previous observations (Figure 4G). Gene Ontology (GO) functional enrichment analysis of the selected genes indicated their participation in synaptic signaling and nervous system development (Figure $4 \mathrm{H}$, Table S2 ). A search for transcription factor binding site motifs within the selected regions revealed the enrichment of factors involved in neuronal processes, including NFR1 (Figure 4I, see Methods for details). NRF1 is involved in neurite growth and its deletion leads to neuronal dysfunctions (Chang et al., 2005; Piao et al., 2012). Another identified factor, MXI1, was reported as a tumor suppressor negatively regulating c-MYC and an inhibitor of glioma proliferation(Lahoz et al., 1994; Manni et al., 2002). Altogether, these findings indicate higher expression of genes related to neuronal signaling in PA tumors when compared to DA/GB gliomas.

\section{Pilocytic astrocytomas show H3K4me3 hypermethylation at the promoters targeted by PRC2}

Hierarchical clustering of samples with $\mathrm{H} 3 \mathrm{~K} 4 \mathrm{me} 3$ marks in promoters shows a noticeable difference between PA and DA/GB gliomas (Figure 5A). The majority of the PA samples exhibited large pairwise overlap of $\mathrm{H} 3 \mathrm{~K} 4 \mathrm{me} 3$ marked promoters (0.93-0.95), while the pairwise overlap between PA samples and DA/GB gliomas was lower. Additionally, the group of DA/GB tumors showed relatively lower similarity among them. We identified 359 genes with the promoters differentially marked with H3K4me3 betweenthe five PA samples forming the tight cluster, and DA/GB gliomas. The vast majority of them (338 out of 359) had the active chromatin mark in their promoters only in PA samples (Table S3) and were characterized by a significant increase in the mRNA levels (Figure 5B), H3K27ac and ATAC-seq signal intensity in PA samples in comparison to DA/GB samples (Figure S5A-B). Interestingly, this promoter set is rich in binding sites of the PRC2 complex components: 
nearly half of them contain the binding sites for EZH2 and nearly one sixth contains the binding sites for SUZ12, based on ChIP-seq data from the ENCODE project (Gerstein et al., 2012). When compared to promoters marked by H3K4me3 in all our samples, the promoters specifically active in PA are enriched in EZH2 and SUZ12 binding sites 7.2 and 2.5 times, respectively (Figure $5 \mathrm{C}$ ). Additionally, transcription factor motifs enrichment analysis showed that the promoters specifically active in PA are characterized by the presence of GC rich sequences, while no strongly enriched motifs were identified (Figure S5C). This observation is in agreement with an expected lack of strong sequence specificity in the PRC2 complex binding. Moreover, when we compared the motifs that are enriched with respect to other active promoters, we found much fewer motifs significantly enriched in the PA specific promoters than when compared to other inactive promoters. Also, comparison to motifs enriched in DA/GB-specific promoters, supports lack of PA-specificity of the GC-rich motifs (Figure S5D).

We acquired the whole-genome bisulfite sequencing data from the same tumor samples as discussed above. Interestingly, we found DNA methylation levels at the promoters with $\mathrm{EZH} 2$ binding sites to be lower in PA compared to DA/GB gliomas (Figure 5D). This observation was corroborated by the data from the independent PA and GB dataset analyzed with HumanMethylation450 BeadChip array (Figure 5E). Overall, these promoters may represent an epigenetic signature which differentiates PA from DA/GB gliomas and indicate that mechanisms related to chromatin repression through the PRC2 complex together with DNA methylation may play leading roles in regulating a set of genes.

Using PANTHER Overrepresentation Test (Mi et al., 2019), we searched for enriched Gene Ontology (GO) terms in a set of the PA-specific genes with EZH2 binding sites in promoters. This analysis did not reveal strong enrichments, except for terms related with developmental processes, cell differentiation, transmembrane transport processes (Figure $5 F$, S5G). For genes specifically active in PA, but without EZH2 binding site in promoters, no significantly enriched GO terms were identified. We speculate that altered expression of these genes may arise as a secondary effect, since representatives of several diverse transcription factor families (including HES, GATA, NR2E, VSX and SIM1) can be identified among those genes that have $\mathrm{EZH} 2$ binding site in their promoters.

Pilocytic astrocytomas are usually located in cerebellum, while higher grade gliomas more often develop in cortical areas (Larjavaara et al., 2007), we set out to verify, whether the observed set of promoters, specifically activated in PA tumors may be connected to the common developmental origin of these tumors. We collected DNA methylation levels of the promoters specifically active in PA and having an EZH2 binding site in previously published samples from different brain regions (Lambert et al., 2013; Pidsley et al., 2014). In agreement with our prediction, these promoters have increased DNA methylation levels in normal cortex compared to cerebellum (Figure S5F), however the increase is not as 
significant as in our PA samples. Similarly, PA located in infratentorial brain regions are hypermethylated compared to the control cerebellum (Figure S5G). These differences in DNA methylation are accompanied by changed transcript expression levels (Figure $\mathrm{S} 5 \mathrm{H}$ ) supporting the functional nature of the hypermethylation. These results suggest that the epigenetic mechanism of activation of the genes in question is a combination of their developmental background and further tumor-related dysregulation of the PRC2 complex. At the same time, these genes exhibit even higher methylation levels in GB samples, consistent with expected dysregulation of Polycomb complexes in high grade tumors.

\section{Differential activation of regulatory regions coupled with chromatin contacts uncovers the FOXM1-ANXA2R network operating in glioblastoma and impacting patient survival}

Chromatin conformation may influence regulation of gene expression (Lupiáñez et al., 2015, Donaldson-Collier et al., 2019) and topologically associating domains (TADs) segregate the genome into megabase-scale regions. Enhancers and their cognate promoters are typically located in the same TAD, even if their genomic distance between them is as large as a mega-base (Racko et al., 2018). Since we were most interested in promoter-enhancer contacts that require high-resolution maps, we used published high resolution fetal brain $\mathrm{Hi}-\mathrm{C}$ data (Won et al., 2016) to putative enhancers locations to search for potential interactions that may elicit pathogenic mechanisms. In order to validate that these data are applicable to putative glioma enhancers, we verified that the TAD boundaries in the fetal brain data are not significantly different from those derived from low-resolution glioma Hi-C data (Harewood et al. 2017)

We identified 109 enhancer-promoter pairs with significant contact frequency based on $\mathrm{Hi}-\mathrm{C}$ data and a high correlation between the enhancer coverage in H3K27ac ChIP-seq data and transcript expression (Spearman $\mathrm{R}>0.7$; Figure $6 \mathrm{~A}$ ). The identified contacts corresponded to 85 genes among which 17 (FDR < 0.01) were differentially expressed in tumors of different malignancy (Figure $6 \mathrm{~B}$ ). We focused on genes whose expression levels were the highest in GB samples and the lowest in PA samples. We obtained a list of 5 genes, and for all of them we found a significant correlation between promoter and putative enhancer activity levels (Figure $6 \mathrm{C}$ ). One of the identified genes, $A N X A 2 R$, encodes a receptor for Annexin A2 (ANXA2), an element of ANXA2-ANXA2R axis known to play a role in cancerogenesis by promoting cell invasion and migration (D'Souza et al., 2012; Shiozawa et al., 2008). Annexin 2 is the most abundant protein in breast cancer-derived exosomes and enhances angiogenesis (Maji et al., 2017).

The analysis of the putative enhancer sequence revealed a presence of several transcription factor binding motifs, including motifs for the FOXM1 transcription factor (Figure S6]. FOXM1 expression was significantly increased in malignant gliomas: DA and GB (Figure 6D). Comparison of FOXM1 and ANXA2R RNA-seq profiles showed a highly significant positive correlation between their expression levels in 33 samples from our cohort (Figure 6E) and in 299 TCGA glioma samples (Figure 6F). This high level of co-expression 
between FOXM1 and ANXA2R indicates a presence of a regulatory network involving these two genes. To further explore the association between their expression levels and the patient's predicted outcome, we performed survival analyses stratifying patients according to ANXA2R or FOXM1 expression levels (Figure 6G) using TCGA datasets. For both genes, patients with higher expression levels were associated with shorter survival, even when patients with DA and GB were not combined (Figure S6). This shows that the identified $A N X A 2 R$ regulation may be clinically relevant.

To validate experimentally the discovered regulatory link, we evaluated $A N X A 2 R$ and FOXM1 expression levels in different glioma cells: LN18, LN229, U87 established glioma cell lines, patient-derived WG4, IPIN glioma cell cultures (Ciechomska et al., 2016) and normal human astrocytes (NHA) using quantitative PCR (qPCR). Relatively to normal human astrocytes, the expression of $A N X A 2 R$ in those cells was elevated only in patient-derived WG4 glioma cells; while FOXM1 expression was significantly elevated in 4 out of 5 cell lines (Figure $6 \mathrm{H}$ ). For further studies we have selected WG4 glioma cells due to the highest ANXA2R and FOXM1 expression in those cells.

In order to test whether the predicted enhancer-promoter contact occurs in the context of glioma cells, we performed a Capture-C assay on WG4 cells, retrieving the gene promoter cleavage fragment with all interacting DNA fragments. The profile obtained by plotting the data as a function of chromosome 5 genomic location (Figure 6I) shows a pattern similar to the one that can be obtained with $4 \mathrm{C}$-seq. The coverage plot shows enrichment in reads around the studied enhancer denoted by a purple arrow (Figure 6I), confirming occurrence of the enhancer-promoter contact in glioma cells. As illustrated in Figure 6J, FOXM1 binds to the CCNB1 gene promoter in WG4 cells (CCNB1 encodes CYCLINB1 and is a known FOXM1 target in cancer cells) as demonstrated by ChIP-qPCR. FOXM1 binds to both the promoter and distal regulatory region of the ANXA2R gene, although only binding to the promoter is statistically significant. Binding of FOXM1 to the $A N X A 2 R$ gene was corroborated with two antibodies from independent suppliers. These results confirm that the predicted chromosomal contact in the ANXA2R locus is consistent with the interaction network obtained with Capture-C and validated by ChIP-qPCR.

\section{DISCUSSION}

Accessible chromatin across the genome reflects a network of permitted interactions through which enhancers, promoters, insulators and chromatin-binding factors cooperatively regulate gene expression. We combined several methods using massively parallel DNA sequencing to assay chromatin accessibility, active or repressive modification of histones to map regulatory sites instructive for gene expression in gliomas. Gliomas arise from neural stem cells, progenitors or dedifferentiated nervous system cells (Azzarelli et al, 2018). It is expected to find active chromatin regions characteristic of the tissue from which tumor originated as well as tumor specific activity in the data obtained from the bulk tumor material. 
By combining acquired data, we create the Atlas regulatory sites in gliomas encompassing brain-specific regulatory sites. Within the presented Atlas that describes glioma epigenome features we discriminated two categories of identified regulatory regions: common and variable. The dataset integrating cis-acting regulatory elements which are commonly present in our cohort tend to exhibit the brain-specific signature supported by evolutionary conservation analysis and intersection with publicly available data for non-brain cells. Taking this into account, our Atlas can serve as a tool not only for assessment of gliomagenesis related events, it can also be beneficial in the field of neuropsychiatric disorders research giving the evidence of biologically relevant role of sites overlapping with GWAS-identified SNPs (Liu et al., 2019).

While the utility of the mapped common promoters and enhancers is broader than just its application to the glioma samples, our study has uncovered also a large universe of distal regions that are surprisingly variable in their activity between samples from different glioma grades. As we have shown in this study, many of these, previously unannotated regions can be linked to their likely target genes using $\mathrm{Hi}-\mathrm{C}$ data from publicly available datasets, obtained from both glioma and fetal brain samples. Such mapping can be very useful in studying biological mechanisms operating in gliomas of different grades, as exemplified in our analysis of the ANXA2R enhancer. The putative enhancer sequence showed a presence of several transcription factor binding motifs, including motifs for a FOXM1 transcription factor. The expression of FOXM1 and ANXA2R RNA-seq profiles showed a strong correlation between our samples and in 299 independent TCGA glioma samples. Importantly, their higher expression levels are associated with the shorter patient survival. We validated the interactions using ChIP-qPCR and Capture-C assay. ANXA2ANXA2R axis known to play a role in cancerogenesis by promoting cell invasion and migration (D'Souza et al., 2012; Shiozawa et al., 2008) While its role on glioblastomas needs to be elucidated, an inverse correlation of its expression with patient survival indicates clinical relevance.

We interrogated the genome-wide maps of open chromatin and modified histones correlating with transcriptional activity to find regulatory networks distinct in tumors of various grades. We showed significant differences between the H3K27ac and $\mathrm{H} 3 \mathrm{~K} 4 \mathrm{me} 3$ profiles in PA and DA/GB samples. We found significantly higher H3K27ac levels in DA/GBs versus PA globally, but a detailed analysis uncovered a group of genes with H3K27ac levels higher in the PAs, contrary to the global trend. Closer inspection of these genes showed that they are characterized by neuronal functions (synaptic transmission and signaling, nervous system development, AMPA receptor activity), reflecting higher contribution of non-transformed neural compartments in PAs. A set of transcription factor binding site motifs indicates potential regulation of these genes by transcription factors such as NRF1, REST, E2F1/4 CTCF, MAX. NRF1 encodes Nuclear Respiratory Factor 1, a transcription factor that activates the expression of mitochondrial oxidative metabolism genes (Johar et al., 2013) that are critical for the maintenance of neuronal homeostasis. E2F2, and E2F4 are 
transcription factors that control numerous target genes, playing a role in cell-cycle progression and apoptosis, with E2F4 acting as a transcriptional repressor (Crosby and Almasan, 2004). The Myc/Max/Mad network depending on composition of Myc-Max or MadMax complexes directs gene-specific transcriptional activation or repression (Grandori et al., 2000). Unlike higher-grade gliomas, which usually exhibit multiple driver mutations (Network et al., 2008), most PAs exhibit a single driver somatic genetic alteration, leading to activation of the MAPK pathway related to FGFR or NTRK2 (Jones et al., 2013) or with rearrangements generating the KIAA1549-BRAF fusion oncogene accounting for $\sim 70 \%$ of PAs (Jones et al., 2008). A recent single cell sequencing of PAs indicates a higher proportion of mature glia-like cells to progenitor-like cells in PAs as compared to other gliomas (Reitman et al., 2019).

In majority of PA samples we identified a set of $\mathrm{H} 3 \mathrm{~K} 4 \mathrm{me} 3$ marked promoters which are characterized by a significant increase in the transcript levels, H3K27ac and ATAC-seq signal intensity in comparison to DA/GB samples (Figure 5B, Figure S5). This promoter set is strongly enriched in binding sites for the PRC2 complex components: EZH2 and SUZ12 proteins according to ChIP-seq data from the ENCODE project (Gerstein et al., 2012). These are likely to be differentiation related genes, consistently with the known dysregulation of $\mathrm{EZH} 2$ and its regulatory networks in high grade gliomas that are characterized by abundance of dedifferentiated glioma stem cells (Jin et al., 2017).

Altogether, we present here the results that provide the first resource integrating so many different types of data on epigenetic and regulatory profiles in gliomas of various grades. We showed several novel findings based on this resource, but we are confident that this resource will pave the way to the potential future strategies in the treatment of gliomas.

\section{Figure Legends}

Figure 1. Experimental workflow

\section{Figure 2 Construction of an atlas of regulatory sites in gliomas.}

(A) Numbers of samples from gliomas of different grades for which ATAC-seq, ChIP-seq and RNA-seq data were collected.

(B) Representative peaks of ATAC-seq, ChIP-seq, RNA-seq at the NRXN2 gene in pilocytic astocytomas (PA04) and glioblastoma (GB01) samples. Note high expression of the NRXN2 in PA in comparison to $G B$, which correlates with the lack of the ATAC-seq signal in GB (shadowed blocks).

(C) Total numbers of peaks identified in all samples across performed experiments.

(D) Numbers of identified common and variable active regulatory elements, genes and transcripts. 
(E) Example of promoter-enhancer interactions identified using Hi-C data (Won et al., 2016). Brown dots represent "anchors", while brown horizontal lines depict predicted interaction sites.

Figure 3. Global characterization of chromatin structure and its relationship with gene expression.

(A) Commonality of histone marks, open chromatin regions and gene expression patterns between samples. Color scale represents the percentage of patients sharing a particular genomic element status. Bar height represents a number of genomic elements (scaled to $100 \%)$.

(B) Venn diagram shows intersection of the promoter regions marked by H3K4me3, H3K27ac and open chromatin sites detected with ATAC-seq.

(C) Evolutionary conservation of the identified promoters (green) and enhancers (purple) measured by PhastCons 100-Way scores. Mean scores for the identified regulatory regions are compared to mean scores obtained for random genomic intervals. P-values were calculated with one-sided Mann-Whitney $U$ test.

(D) Correlation between H3K4me3 (green), H3K27ac (purple) and transcript expression. Normalized ChIP-seq coverage is plotted against an expression level of transcripts of protein coding genes, divided into quantiles.

(E) Hierarchical clustering of samples based on gene expression profiles. Shades of blue indicate tumor grade (from light $=$ normal brain to dark = Grade IV and black PG). In the table on the right side the subtype of glioblastoma samples (GBM) is reported as well as assignment of glioma samples to one of Pan Glioma RNA Expression Clusters ( $L G r)$ as well as IDH1 gene mutation status (IDH).

(F) Correlation between H3K27ac ChIP-seq coverage on enhancers and transcript expression. Numbers above bars indicate numbers of enhancers in each group.

Figure 4 Chromatin activity profiles indicate presence of normal brain signature in pilocytic astrocytomas

$(A, B)$ Scatter-plots representing abundance of the H3K4me3 $(A)$ and H3K27ac (B) marks obtained for PAs (X-axes) and DA/GBs (Y-axes). A single point on the scatter-plots represents an average abundance (ChIP-input) of a corresponding histone mark around transcription start site [TSS+/-2kb]. P-values shown above the plots were estimated with the Wilcoxon rank-sum test.

(C) Comparison of abundance values shown on Figure 4A (X-axis) and 4B (Y-axis). The dashed frame indicates the strongest difference in H3K27ac abundance (top 10\%) values.

(D) Heatmaps showing H3K27ac abundance around the TSS for the top 10\% regions (each row corresponds to a TSS, sorted by signal abundance)

(E) ATAC-seq profiles of 4 PA samples and 4 DA/GB samples (see Methods for details) around TSS overlap with the regions selected on Figure $4 \mathrm{C}$. The blue and red lines 
correspond to PA and DA/GB samples respectively.

(F) Average H3K27ac profiles around TSS. The blue and red lines correspond to the top $10 \%$ regions selected in Figure $4 \mathrm{C}$ and were computed for the PA and DA/GB samples respectively. The gray lines correspond to regions not selected on Figure $4 \mathrm{C}$. The thick lines correspond to the average profiles, while colored areas give reference of the confidence interval for mean $(\mathrm{Cl})$.

(G) Differences of the gene expression levels computed either for genes corresponding to the top $10 \%$ selected regions (left boxplot) or to the rest of the regions (right boxplot). Pvalue shown above the plot and indicating differential regulation was estimated with the Wilcoxon rank-sum test.

(H) Results of Gene Ontology over-representation analysis performed for the genes corresponding to the top $10 \%$ regions. The barplot shows scaled Bonferroni corrected $p$ values and the vertical, dashed red line stands for the significance threshold $(P v=0.05)$.

(I) Logos of top DNA-binding motifs enriched in the top $10 \%$ regions. The enrichment was computed with regard to the rest of the analyzed regions. P-values indicated above the logos stand for the significance of the enrichment and were computed with a tool from MEMESuite (see Methods for details).

\section{Figure 5. A group of promoters targeted by PRC2 exhibits H3K4me3 hypermethylation and DNA hypomethylation in PAs.}

(A) Hierarchical clustering of samples based on the presence of H3K4me3 mark at the promoters. Color scale and numbers on the heatmap indicate similarity between H3K4me3 pattern at the promoters (dark blue, 1 - identical; white, 0 - maximally dissimilar). Red box identified cluster of PA samples.

(B) Expression of transcripts associated with promoters exhibiting H3K4me3 hypermethylation in PAs. Enrichment of binding sites from the ENCODE transcription factor ChIP-seq data in PAs specific promoters compared to promoters active in all samples. The number of peaks for each DNA-binding protein is given in parentheses; enrichment values per megabase are shown next to each bar and indicated with proportional coloring (red highest, blue-lowest).

(C) Enrichment of binding sites from the ENCODE transcription factor ChIP-seq data in PAs specific promoters compared to promoters active in all samples. The number of peaks for each DNA-binding protein is given in parentheses; enrichment values per megabase are shown next to each bar and indicated with proportional coloring (red - highest, blue-lowest).

(D) DNA methylation in promoters exhibiting $\mathrm{H} 3 \mathrm{~K} 4 \mathrm{me} 3$ hypermethylation in PAs and having an EZH2 binding site. Methylation levels were determined with WGBS. Samples with IDH mutations were excluded from the plot.

(E) DNA methylation in promoters exhibiting H3K4me3 hypermethylation in PAs and having

an EZH2 binding site in an independent cohort, determined by HumanMethylation450 BeadChip array.

(F) GO terms enriched in the set of the PA-specific genes with EZH2 binding sites in 
promoters. The enrichment was calculated using the PANTHER Overrepresentation Test (Mi et al., 2019).

Figure 6. Intersection of the Atlas data with fetal brain Hi-C data reveals FOXM1ANXA2R network operating in glioblastoma and impacting patients survival.

(A) A graphical representation of the chromatin contacts identified based on fetal brain Hi-C data.

(B) A heatmap shows normalized average expression levels of 17 differentially expressed genes (FDR < 0.01) that were selected in the contact analysis (Figure 6A, see Methods for details). Genes which expression levels were increased in high grade gliomas are depicted.

(C) A barplot shows significance of enhancer-promoter co-activation for the 5 genes shown in Figure 6B. The co-activation was computed with Pearson's correlation of H3K27ac enrichment in the putative enhancer and $\mathrm{H} 3 \mathrm{~K} 4 \mathrm{me} 3$ enrichment in the corresponding promoter.

(D) Expression levels of FOXM1 gene in glioma samples. The adjusted P-value (FDR) corresponds to differences in expression levels and was computed with edgeR Bioconductor package. Logo showed above the plot represents FOXM1 DNA-binding motif.

$(\mathrm{E}, \mathrm{F})$ Scatterplots show co-expression of FOXM1 (X-axes) and ANXA2R (Y-axes) in our (E) and TCGA $(F)$ data. Gene expression levels are represented as log-transformed FPKMs. Corresponding Pearson's correlation coefficients are shown above the plots. Tumor grades were color-coded.

(G) Kaplan-Meier curves plotted for the TCGA data. The patients were stratified by either by ANXA2R (top part) or by FOXM1 (bottom part) expression levels into three equinumerous sub-groups. Differences in survival rates between sub-groups were assessed with the logrank test.

(H) ANXA2R and FOXM1 mRNA expression in normal human astrocytes (NHA), glioma patient derived cell cultures (WG4 and IPIN) and established glioma cell lines (LN229, LN18, U87) was evaluated by RT-qPCR. Data were normalized to the expression of GAPDH mRNA determined in the same sample. $P$ values were considered significant when ${ }^{*} P<0.05$ and ${ }^{* *} P<0.01$ (t-test).

(I) A coverage plot of WG4 Capture-C profile representing interactions around the viewpoint, ANXA2R promoter fragment, marked with a green arrow. Putative enhancer is denoted by purple arrow indicating region enriched in fragment reads. Fragment-based raw data is visualized as grey dots, whereas blue dots represent smoothed data. The trend line is blue whereas quantiles are shown as light-grey bands.

(J) ChIP-qPCR analysis of FOXM1 binding to the CCNB1 gene promoter (left part) that serves as a positive control, and to the $A N X A 2 R$ proximal and distal regulatory regions (middle part). Results are calculated as $\%$ of input, mean \pm SD $(n=3)$ and compared to lgG. T-test was used for statistical analysis. Antibodies from two different companies showed around 2-fold enrichment in FOXM1 binding to the $A N X A 2 R$ gene promoter over a neutral $\lg G$ 


\section{Acknowledgments}

This work was supported by Polish National Science Centre grant DEC-2015/16/W/NZ2/00314] and by the Foundation for Polish Science TEAM-TECH Core Facility project "NGS platform for comprehensive diagnostics and personalized therapy in neuro-oncology" (BW, BG). We would like to thank Marcin Kruczyk for his contributions to the concept of the study, Luca Giorgetti and Mariya Kryzhanovska for their help in performing the Capture- $C$ experiments and Jacek Koronacki for comments on the manuscript.

\section{Author Contributions}

Conceptualization: JK BK MJD KD BWi KS; Methodology: BK, KS, Investigation in the lab: KS, RG, SK, BG; Computational investigation: BWo, MM, AMac, JM, AD, IG, HK, KS, MD, MJD, MJ, BWi; Resources: KK, WG, MR, TC AMar, Writing - Original draft: KS, JM, MM, BK, BWi; Writing - Review \& editing: BWi, BK, KS, MJD, MM, KD, JK. All authors have read and approved the final version of the manuscript.

\section{Declaration of Interests}

The authors declare no competing interests.

\section{References}

Azzarelli, Roberta, Benjamin D. Simons, and Anna Philpott. "The developmental origin of brain tumours: a cellular and molecular framework." Development 145.10 (2018). Bernstein, B.E., Mikkelsen, T.S., Xie, X., Kamal, M., Huebert, D.J., Cuff, J., Fry, B., Meissner, A., Wernig, M., Plath, K., et al. (2006). A Bivalent Chromatin Structure Marks Key Developmental Genes in Embryonic Stem Cells. Cell 125, 315-326.

Bernstein, B.E., Meissner, A., and Lander, E.S. (2007). The Mammalian Epigenome. Cell $128,669-681$.

Bogdanovic, O., Fernandez-Minan, A., Tena, J.J., de la Calle-Mustienes, E., Hidalgo, C., van Kruysbergen, I., van Heeringen, S.J., Veenstra, G.J.C., and Gomez-Skarmeta, J.L. (2012). Dynamics of enhancer chromatin signatures mark the transition from pluripotency to cell specification during embryogenesis. Genome Res. 22, 2043-2053.

Bonn, S., Zinzen, R.P., Girardot, C., Gustafson, E.H., Perez-Gonzalez, A., Delhomme, N., Ghavi-Helm, Y., Wilczyński, B., Riddell, A., and Furlong, E.E.M. (2012). Tissue-specific analysis of chromatin state identifies temporal signatures of enhancer activity during embryonic development. Nat. Genet. 44, 148-156.

Cadieux, B., Ching, T.T., VandenBerg, S.R., and Costello, J.F. (2006). Genome-wide hypomethylation in human glioblastomas associated with specific copy number alteration, methylenetetrahydrofolate reductase allele status, and increased proliferation. Cancer Res. $66,8469-8476$.

Ceccarelli, M., Barthel, F.P., Malta, T.M., Sabedot, T.S., Salama, S.R., Murray, B.A., 
Morozova, O., Newton, Y., Radenbaugh, A., Pagnotta, S.M., et al. (2016). Molecular Profiling Reveals Biologically Discrete Subsets and Pathways of Progression in Diffuse Glioma. Cell 164, 550-563.

Chang, W.-T., Chen, H., Chiou, R.-J., Chen, C.-Y., and Huang, A.-M. (2005). A novel function of transcription factor alpha-Pal/NRF-1: increasing neurite outgrowth. Biochem. Biophys. Res. Commun. 334, 199-206.

Ciechomska, I.A., Przanowski, P., Jackl, J., Wojtas, B., and Kaminska, B. (2016). BIX01294, an inhibitor of histone methyltransferase, induces autophagy-dependent differentiation of glioma stem-like cells. Sci. Rep. 6, 38723.

Crosby, M.E., and Almasan, A. (2004). Opposing roles of E2Fs in cell proliferation and death. Cancer Biol. Ther. 3, 1208-1211.

D'Souza, S., Kurihara, N., Shiozawa, Y., Joseph, J., Taichman, R., Galson, D.L., and Roodman, G.D. (2012). Annexin II interactions with the annexin II receptor enhance multiple myeloma cell adhesion and growth in the bone marrow microenvironment. Blood 119, 18881896.

Fontebasso, A.M., Liu, X.-Y., Sturm, D., and Jabado, N. (2013). Chromatin Remodeling Defects in Pediatric and Young Adult Glioblastoma: A Tale of a Variant Histone 3 Tail. Brain Pathol. 23, 210-216.

Fullard, J.F., Giambartolomei, C., Hauberg, M.E., Xu, K., Voloudakis, G., Shao, Z., Bare, C., Dudley, J.T., Mattheisen, M., Robakis, N.K., et al. (2017). Open chromatin profiling of human postmortem brain infers functional roles for non-coding schizophrenia loci. Hum. Mol. Genet. 26, 1942-1951.

Gerstein, M.B., Kundaje, A., Hariharan, M., Landt, S.G., Yan, K.K., Cheng, C., Mu, X.J., Khurana, E., Rozowsky, J., Alexander, R., et al. (2012). Architecture of the human regulatory network derived from ENCODE data. Nature 489, 91-100.

Grandori, C., Cowley, S.M., James, L.P., and Eisenman, R.N. (2000). The Myc/Max/Mad Network and the Transcriptional Control of Cell Behavior. Annu. Rev. Cell Dev. Biol. 16, 653-699.

Harewood, Louise, Kamal Kishore, Matthew D. Eldridge, Steven Wingett, Danita Pearson, Stefan Schoenfelder, V. Peter Collins, and Peter Fraser. "Hi-C as a tool for precise detection and characterisation of chromosomal rearrangements and copy number variation in human tumours." Genome biology 18, no. 1 (2017): 125.

Jin, X., Kim, L.J.Y., Wu, Q., Wallace, L.C., Prager, B.C., Sanvoranart, T., Gimple, R.C., Wang, X., Mack, S.C., Miller, T.E., et al. (2017). Targeting glioma stem cells through combined BMI1 and EZH2 inhibition. Nat. Med. 23, 1352.

Johar, K., Priya, A., Dhar, S., Liu, Q., and Wong-Riley, M.T.T. (2013). Neuron-specific specificity protein 4 bigenomically regulates the transcription of all mitochondria- and nucleus-encoded cytochrome c oxidase subunit genes in neurons. J. Neurochem. 127, 496508. 
Jones, D.T.W., Kocialkowski, S., Liu, L., Pearson, D.M., Bäcklund, L.M., Ichimura, K., and Collins, V.P. (2008). Tandem Duplication Producing a Novel Oncogenic BRAF Fusion Gene Defines the Majority of Pilocytic Astrocytomas. Cancer Res. 68, 8673-8677.

Jones, D.T.W., Hutter, B., Jäger, N., Korshunov, A., Kool, M., Warnatz, H.-J., Zichner, T., Lambert, S.R., Ryzhova, M., Quang, D.A.K., et al. (2013). Recurrent somatic alterations of FGFR1 and NTRK2 in pilocytic astrocytoma. Nat. Genet. 45, 927.

Karlic, R., Chung, H.-R., Lasserre, J., Vlahovicek, K., and Vingron, M. (2010). Histone modification levels are predictive for gene expression. Proc. Natl. Acad. Sci. 107, 29262931.

Kouzarides, T. (2007). Chromatin Modifications and Their Function. Cell 128, 693-705. Lahoz, E.G., Xu, L., Schreiber-Agus, N., and DePinho, R.A. (1994). Suppression of Myc, but not E1a, transformation activity by Max-associated proteins, Mad and Mxi1. Proc. Natl. Acad. Sci. 91, 5503 LP-5507.

Lambert, S.R., Witt, H., Hovestadt, V., Zucknick, M., Kool, M., Pearson, D.M., Korshunov, A., Ryzhova, M., Ichimura, K., Jabado, N., et al. (2013). Differential expression and methylation of brain developmental genes define location-specific subsets of pilocytic astrocytoma. Acta Neuropathol. 126, 291-301.

Larjavaara, S., Mäntylä, R., Salminen, T., Haapasalo, H., Raitanen, J., Jääskeläinen, J., and Auvinen, A. (2007). Incidence of gliomas by anatomic location. Neuro. Oncol. 9, 319-325. Liu, C., Kanazawa, T., Tian, Y., Mohamed Saini, S., Mancuso, S., Mostaid, M.S., Takahashi, A., Zhang, D., Zhang, F., Yu, H., et al. (2019). The schizophrenia genetics knowledgebase: a comprehensive update of findings from candidate gene studies. Transl. Psychiatry 9, 205.

Louis, D.N., Perry, A., Reifenberger, G., Deimling, A. Von, Figarella, D., Webster, B., Hiroko, K.C., Wiestler, O.D., Kleihues, P., and Ellison, D.W. (2016). The 2016 World Health Organization Classification of Tumors of the Central Nervous System : a summary. Acta Neuropathol. 131(6):803-20

Mack, S.C., Singh, I., Wang, X., Hirsch, R., Wu, Q., Villagomez, R., Bernatchez, J.A., Zhu, Z., Gimple, R.C., Kim, L.J.Y., et al. (2019). Chromatin landscapes reveal developmentally encoded transcriptional states that define human glioblastoma. J. Exp. Med. 216, 1071 LP1090.

Maji, S., Chaudhary, P., Akopova, I., Nguyen, P.M., Hare, R.J., Gryczynski, I., and Vishwanatha, J.K. (2017). Exosomal Annexin II Promotes Angiogenesis and Breast Cancer Metastasis. Mol. Cancer Res. 15, 93-105.

Maleszewska, M., and Kaminska, B. (2013). Is glioblastoma an epigenetic malignancy? Cancers (Basel). 5, 1120-1139.

Manni, I., Tunici, P., Cirenei, N., Albarosa, R., Colombo, B.M., Roz, L., Sacchi, A., Piaggio, G., and Finocchiaro, G. (2002). Mxi1 inhibits the proliferation of U87 glioma cells through down-regulation of cyclin B1 gene expression. Br. J. Cancer 86, 477-484.

Network, T.C.G.A.R., McLendon, R., Friedman, A., Bigner, D., Van Meir, E.G., Brat, D.J., M. Mastrogianakis, G., Olson, J.J., Mikkelsen, T., Lehman, N., et al. (2008). Comprehensive 
genomic characterization defines human glioblastoma genes and core pathways. Nature 455, 1061.

Piao, Y., Kim, H.G., Oh, M.S., and Pak, Y.K. (2012). Overexpression of TFAM, NRF-1 and myr-AKT protects the MPP+-induced mitochondrial dysfunctions in neuronal cells. Biochim. Biophys. Acta - Gen. Subj. 1820, 577-585.

Pidsley, R., Viana, J., Hannon, E., Spiers, H., Troakes, C., Al-saraj, S., Mechawar, N., Turecki, G., Schalkwyk, L.C., Bray, N.J., et al. (2014). Methylomic profiling of human brain tissue supports a neurodevelopmental origin for schizophrenia. Genome Biol. 15(10):483

Racko, D., Benedetti, F., Dorier, J., and Stasiak, A. (2018). Are TADs supercoiled? Nucleic Acids Res. 47, 521-532.

Reifenberger, G., Wirsching, H.-G., Knobbe-Thomsen, C.B., and Weller, M. (2016).

Advances in the molecular genetics of gliomas - implications for classification and therapy. Nat. Rev. Clin. Oncol. 14, 434.

Reitman, Z.J., Paolella, B.R., Bergthold, G., Pelton, K., Becker, S., Jones, R., Sinai, C.E., Malkin, H., Huang, Y., Grimmet, L., et al. (2019). Mitogenic and progenitor gene programmes in single pilocytic astrocytoma cells. Nat. Commun. 10, 3731.

Sharma, V., Malgulwar, P.B., Purkait, S., Patil, V., Pathak, P., Agrawal, R., Kulshreshtha, R., Mallick, S., Julka, P.K., Suri, A., et al. (2017). Genome-wide ChIP-seq analysis of EZH2mediated H3K27me3 target gene profile highlights differences between low- and high-grade astrocytic tumors. Carcinogenesis 38, 152-161.

Shiozawa, Y., Havens, A.M., Jung, Y., Ziegler, A.M., Pedersen, E.A., Wang, J., Wang, J., Lu, G., Roodman, G.D., Loberg, R.D., et al. (2008). Annexin II/Annexin II receptor axis regulates adhesion, migration, homing, and growth of prostate cancer. J. Cell. Biochem. 105, 370-380.

Tessarz, P., and Kouzarides, T. (2014). Histone core modifications regulating nucleosome structure and dynamics. Nat. Rev. Mol. Cell Biol. 15, 703.

Tome-Garcia, J., Erfani, P., Nudelman, G., Tsankov, A.M., Katsyv, I., Tejero, R., Zhang, B., Walsh, M., Friedel, R.H., Zaslavsky, E., et al. (2018). Analysis of chromatin accessibility uncovers TEAD1 as a regulator of migration in human glioblastoma. Nat. Commun. 9, 4020.

Won, H., de la Torre-Ubieta, L., Stein, J.L., Parikshak, N.N., Huang, J., Opland, C.K., Gandal, M., Sutton, G.J., Hormozdiari, F., Lu, D., et al. (2016). Chromosome conformation elucidates regulatory relationships in developing human brain. Nature 538, 523-527. 

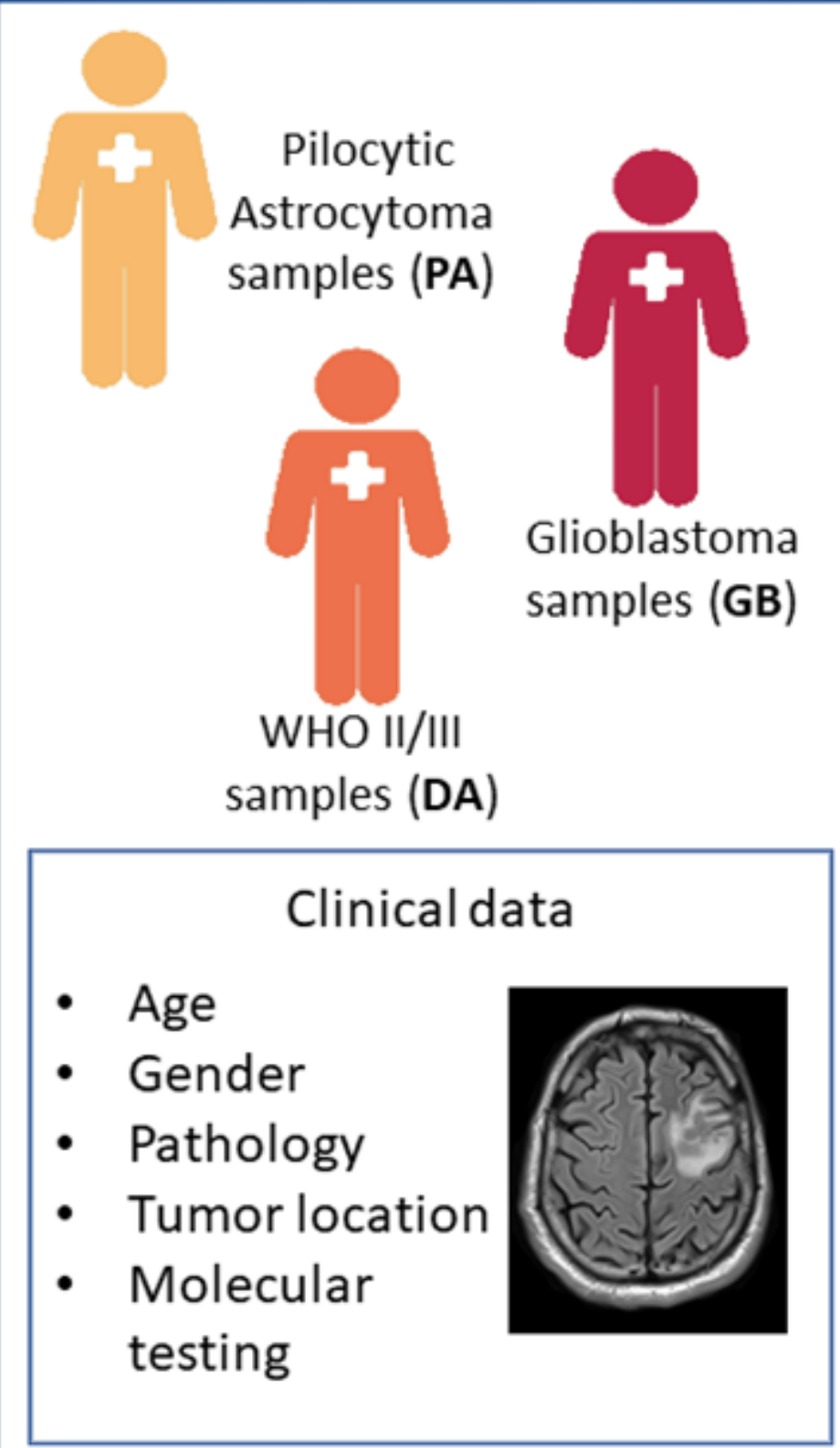

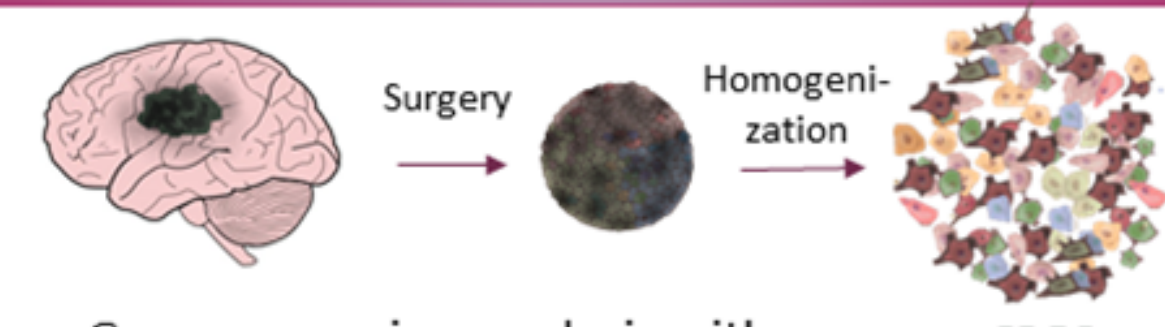

- Gene expression analysis with RNA-seq
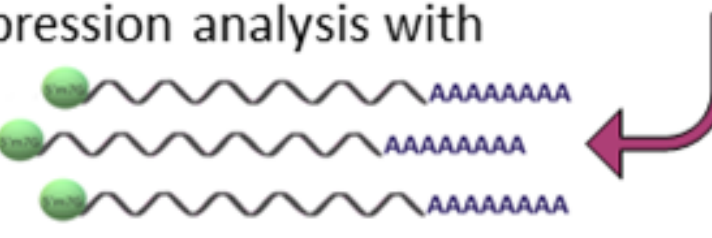

- Open chromatin profiling with ATAC-seq

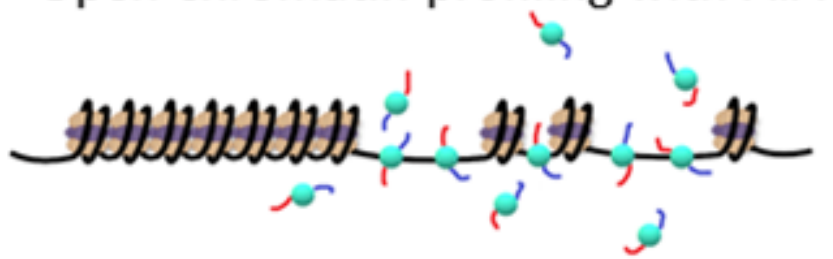

- Chromatin modifications profiling with ChIP-seq

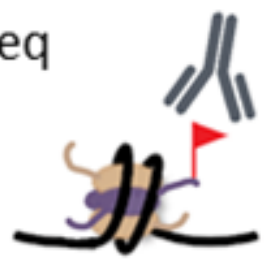

H 3 K4me3

H 3 K27ac

P H3K27me3

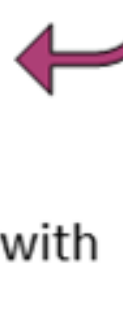

- Genome-wide DNA methylation analysis

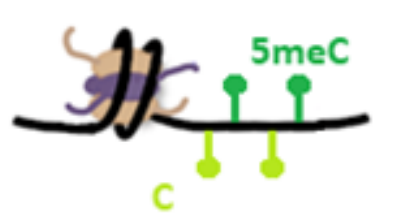

- Genome browser with coverage for each experiment

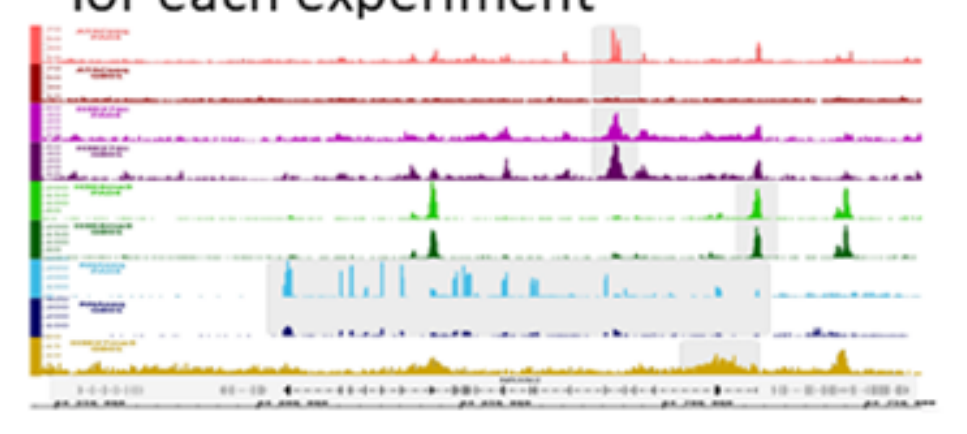

- Transcriptional and epigenomic differential analysis across glioma grades
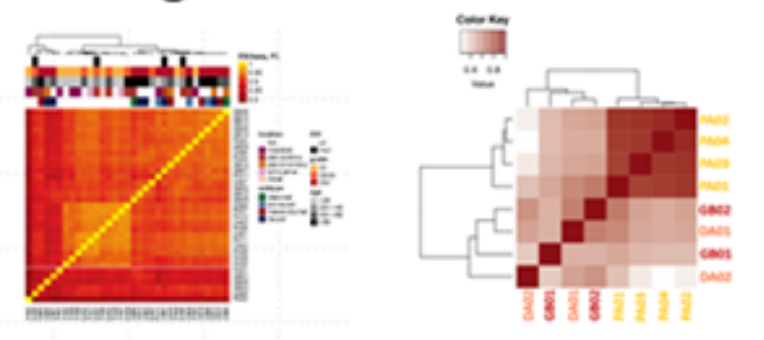

- Identification of regulatory mechanisms behind observed data

$\operatorname{SCC}$ TSOCC. $\mathrm{CSCCACCO}$ c C 

not certified by peer review) is the authorffunder, who has granted bioRxiv a license to display the preprint in perpetuity. It is made

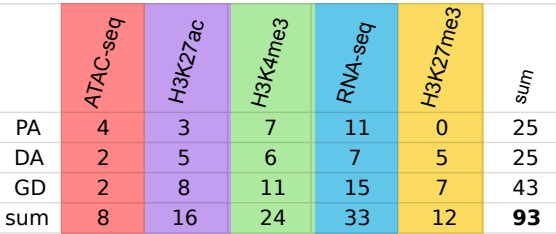

C

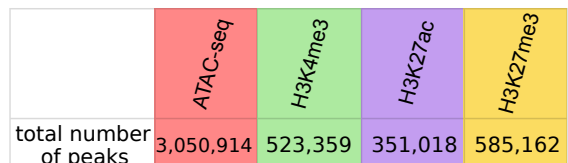

D

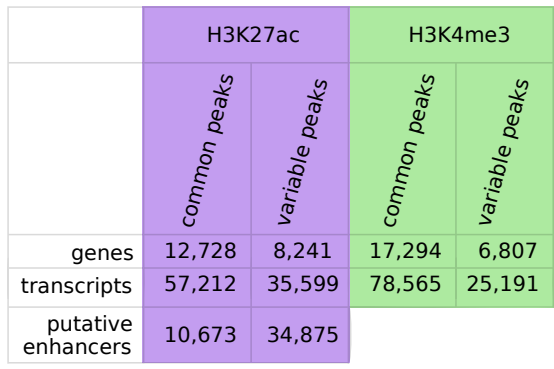

$E$

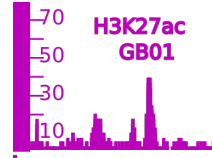

peaks || |

Interactions

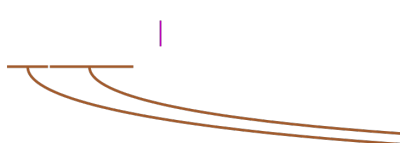

$$
\forall
$$

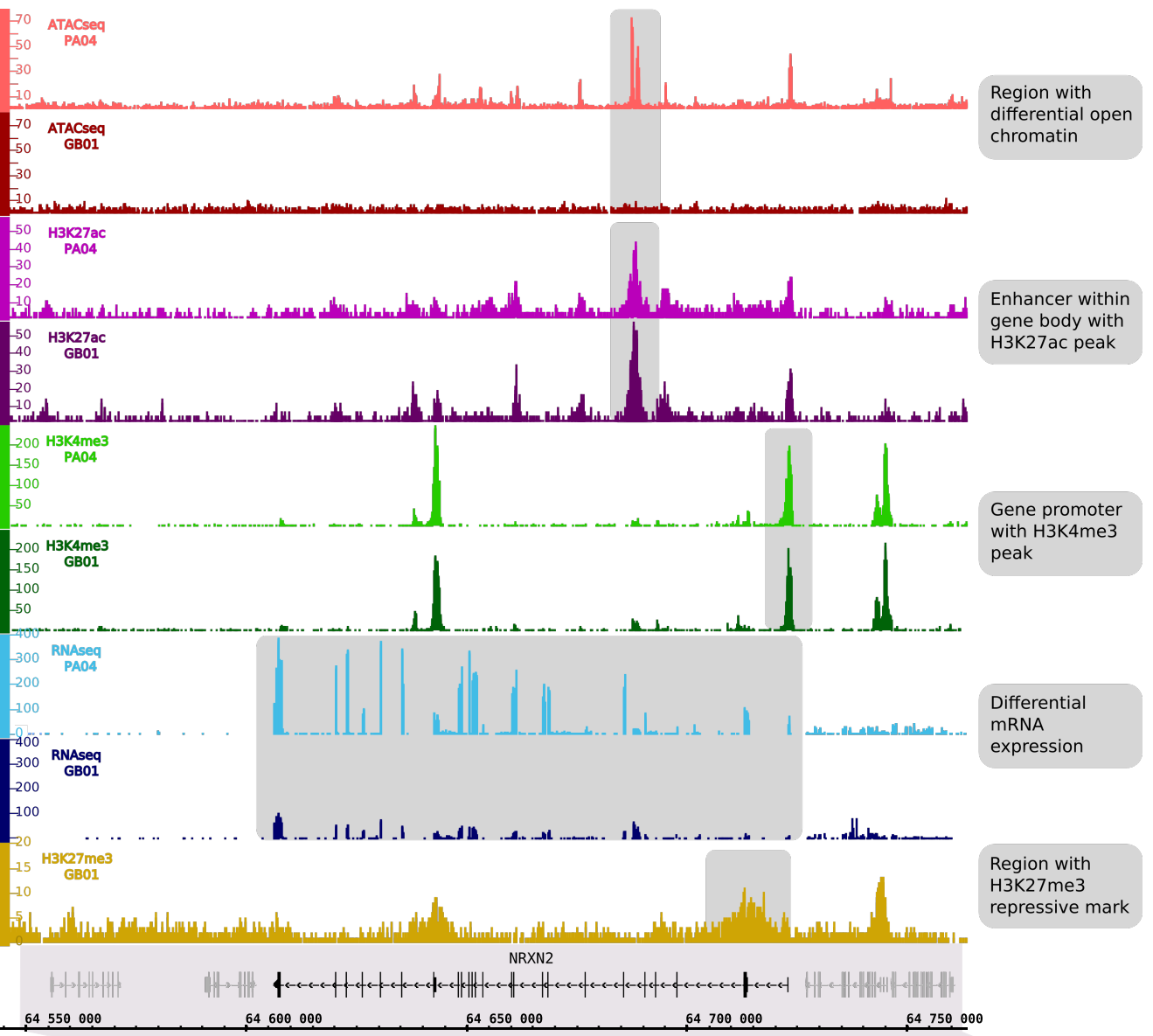


A bioRxiv preprint doi: https://doi.org/10.1101/867861; this version post March 20, 2020. The copyright holder for this preprint (which was not certified by peer review) is the author/funder, who has granted bioRxiv a license to display the preprint in perpetuity. It is made available under aCC-BY-NC-ND 4.0 International license.
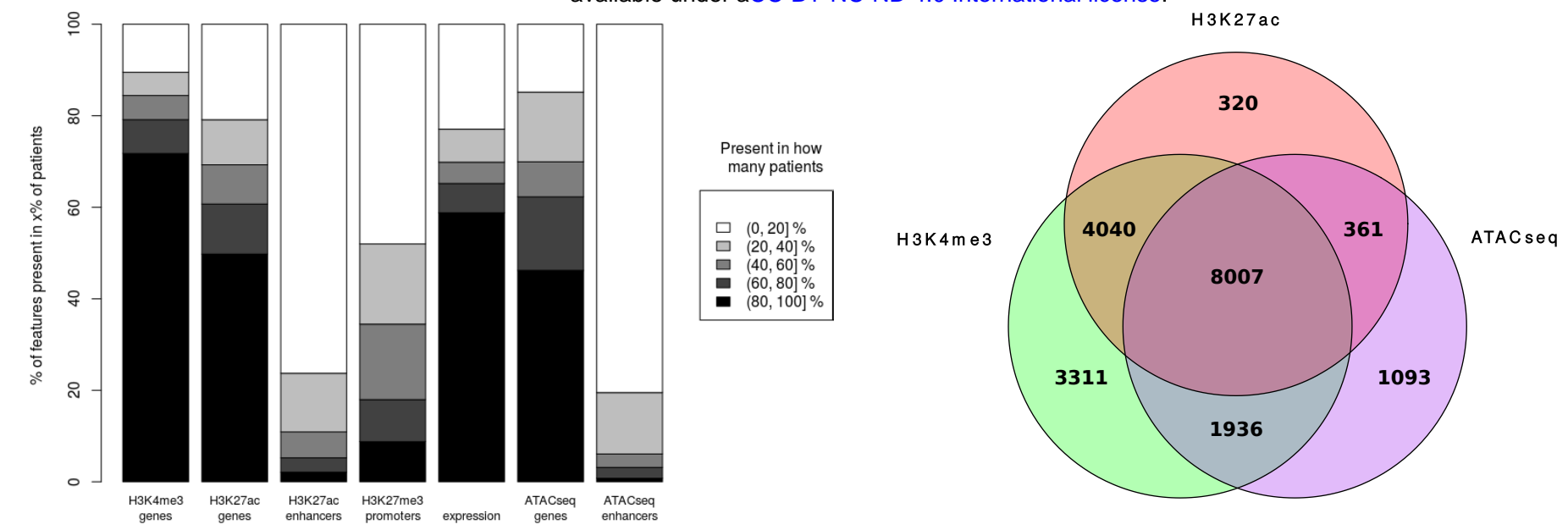

c

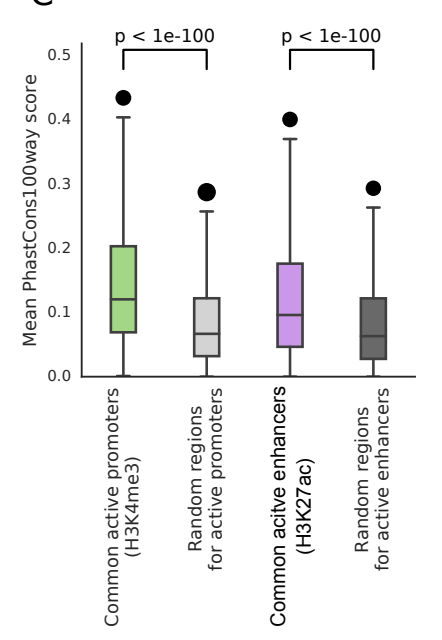

E

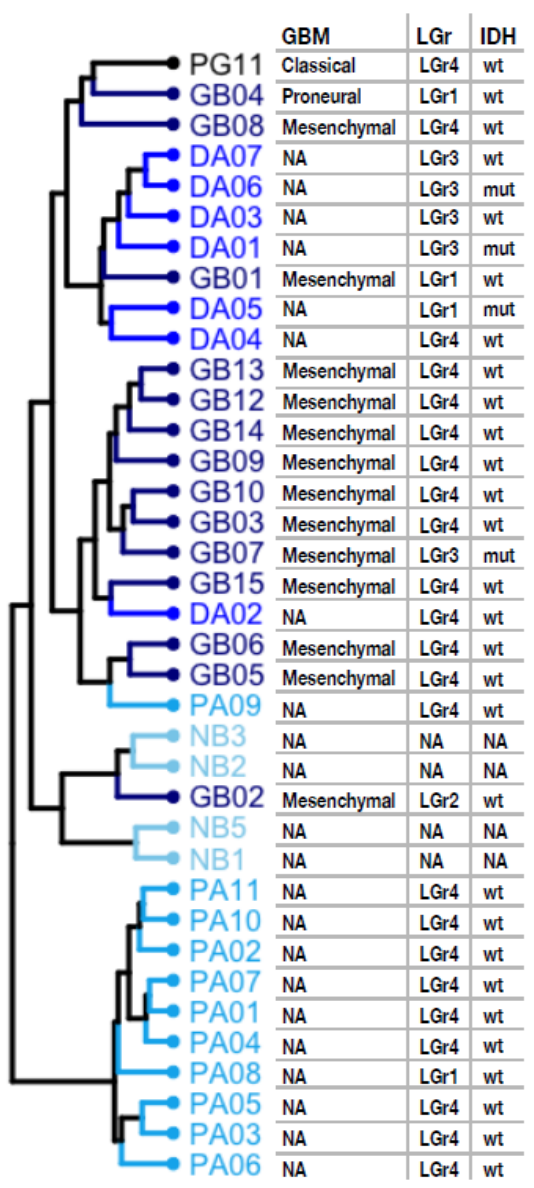

D
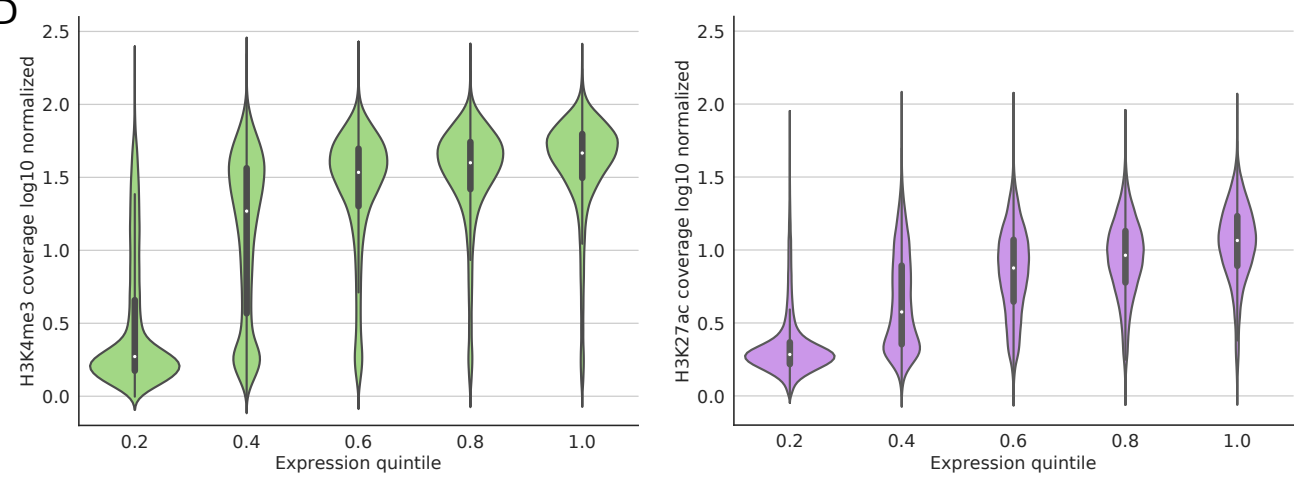

F

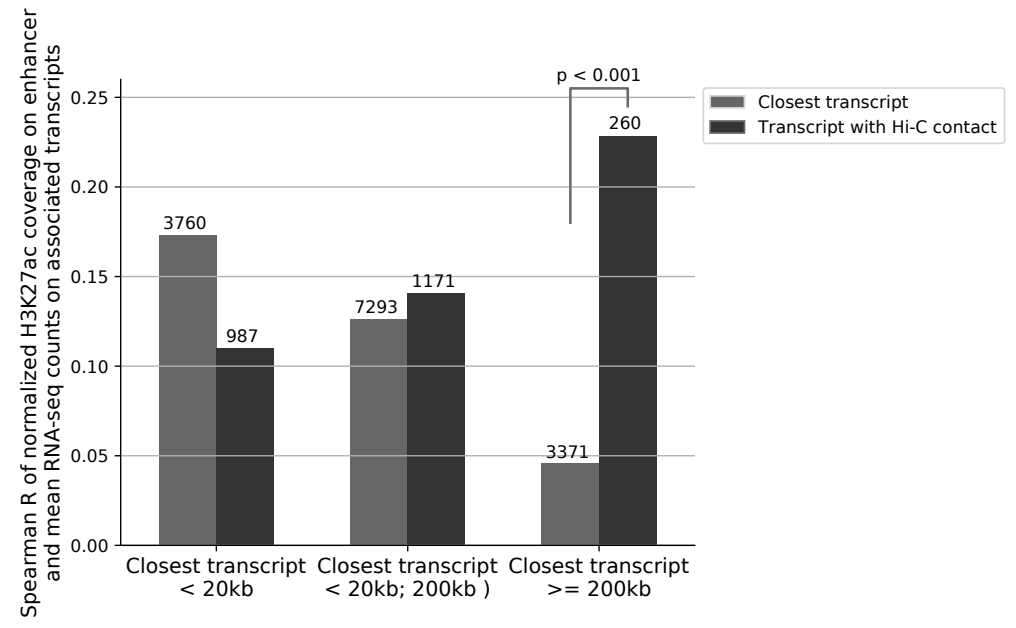


bioRxiv preprint doi: https://doi.org/10.1101/867861; this version posted March 20, 2020. The copyright holder for this preprint (which was

$\mathrm{H} 3 \mathrm{~K} 4 \mathrm{me} 3 \mathrm{TSS} \pm 2 \mathrm{~kb}$, $\mathrm{Pv}<2 \times 10^{-16}$

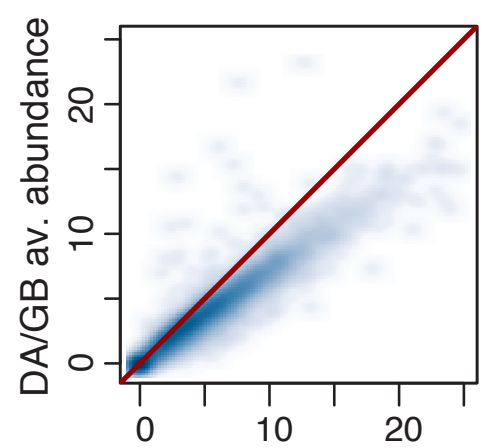

PA av. abundance
$\mathrm{H} 3 \mathrm{~K} 27 \mathrm{ac}$ TSS $\pm 2 \mathrm{~kb}$,

$\mathrm{Pv}<2 \times 10^{-16}$

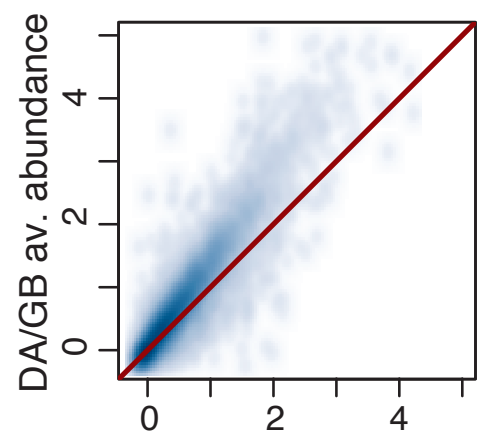

PA av. abundance top $10 \%$ of regions

with higher H3K27ac signal in PA

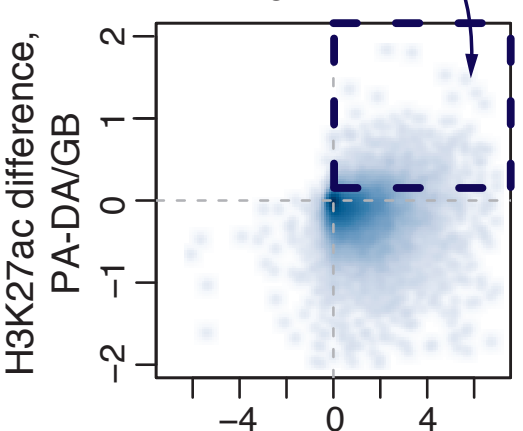

H3K4me3 difference, PA-DA/GB
D

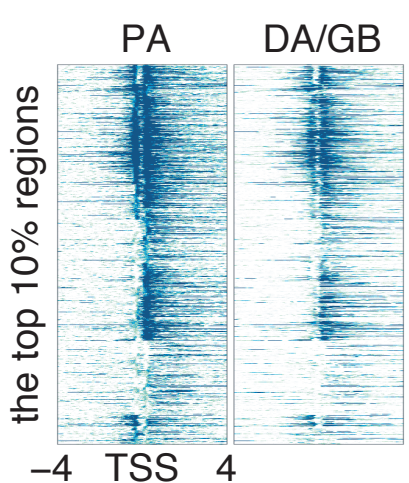

G

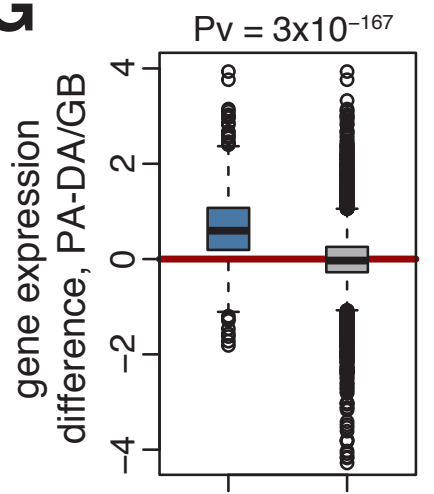

the corresponding the other genes genes

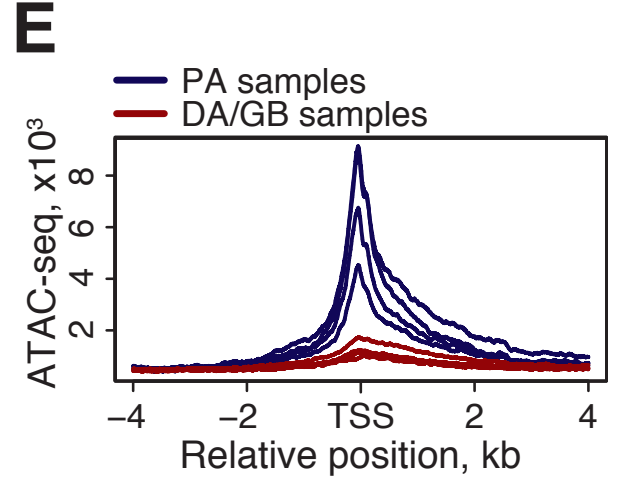

H

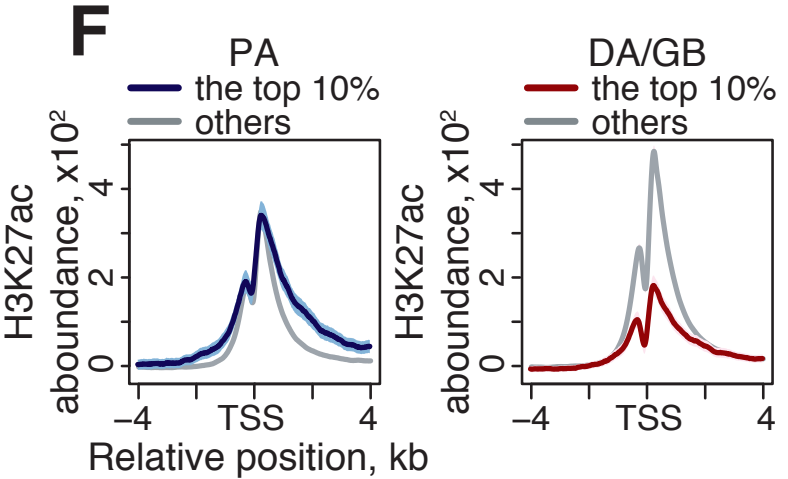

trans-synaptic signaling chemical synaptic transmission anterograde trans-synaptic signaling regulation of trans-synaptic signaling positive regulation of cell-substrate adhesion nervous system development regulation of AMPA receptor activity modulation of chemical synaptic transmission

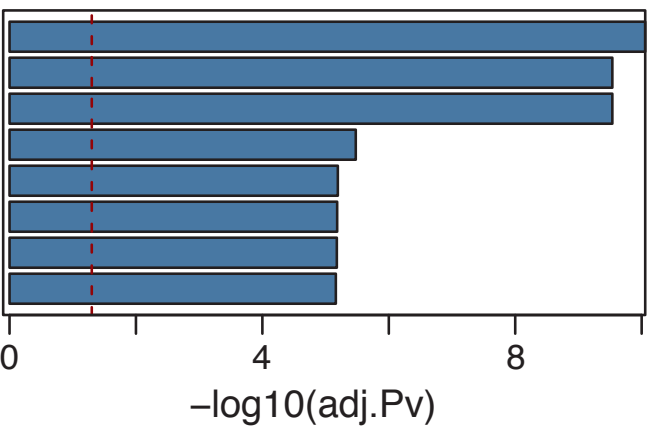

NRF1, Pv $=4 \times 10^{-15} \quad M X I 1, P v=4 \times 10^{-13} \quad Z B B T 7 A, P v=6 \times 10^{-13} \quad$ AP2C, Pv $=10^{-11}$

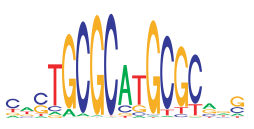

CTCFL, $P v=4 \times 10^{-9}$

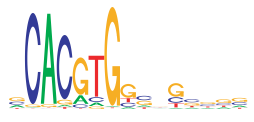
detherice
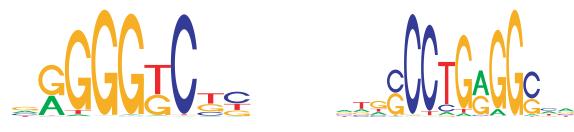

REST, $P v=5 \times 10^{-9}$

$\mathrm{E} 2 \mathrm{~F} 1, \mathrm{PV}=3 \mathrm{X} 10^{-7}$
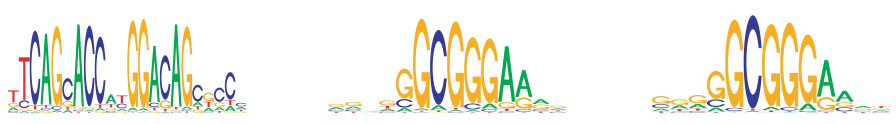

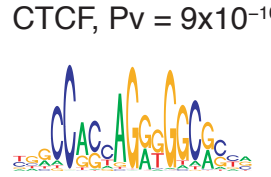

$\mathrm{COE} 1, \mathrm{Pv}=10^{-9}$

$\mathrm{E} 2 \mathrm{~F} 4, \mathrm{Pv}=2 \times 10^{-6}$

$\mathrm{MAX}, \mathrm{Pv}=7 \times 10^{-6}$

AP2A, $P v=9 \times 10^{-6}$
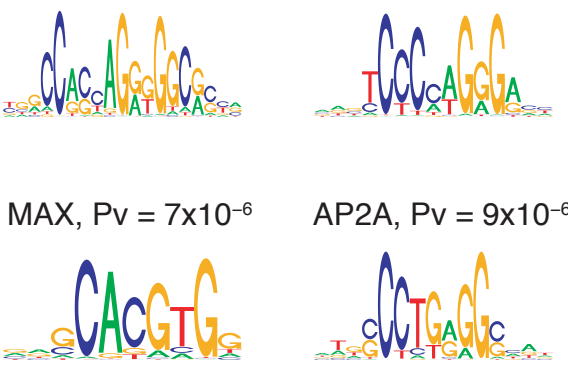
bioRxiv reprint doi: https://doi org/10 1101/867861; this version posted March 20, 2020. The copyright holder for this preprint (which was not certified by peer review) is the author/funder, who has granted bioRxiv a license to display the preprint in perpetuity. It is made

A 0.84

0.78
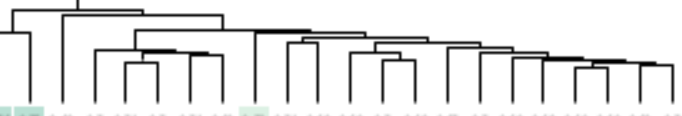

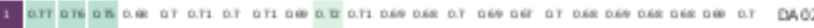

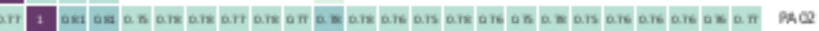

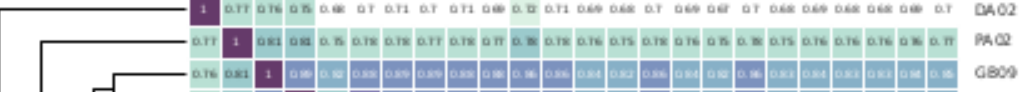

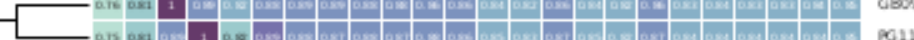

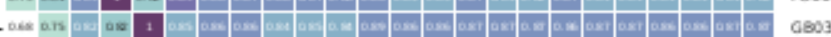

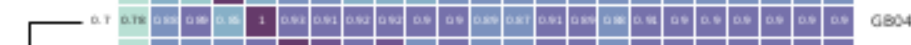

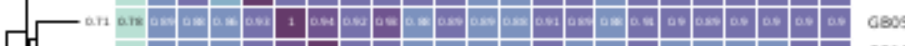

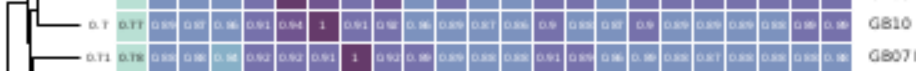

oco on

ors bix

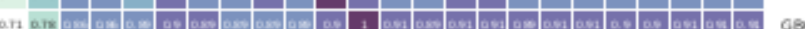

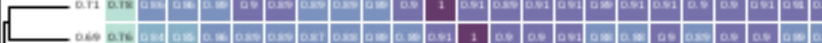

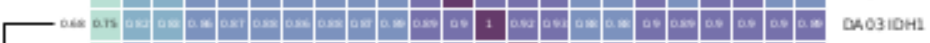

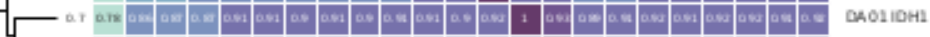

L

osi ans

o.r an

— cus ans

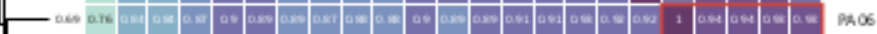

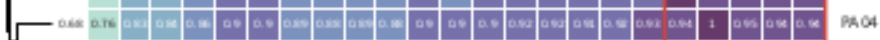

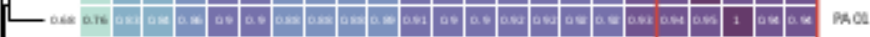

Lis are

o.r an

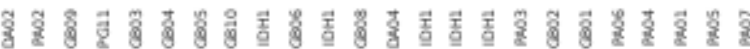 gु}
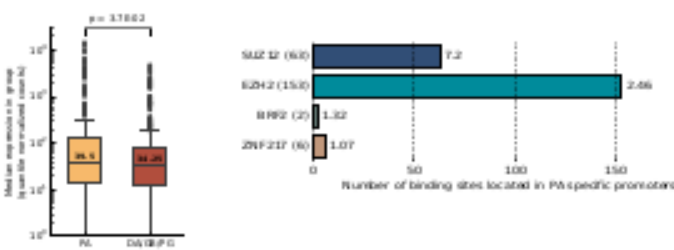

D
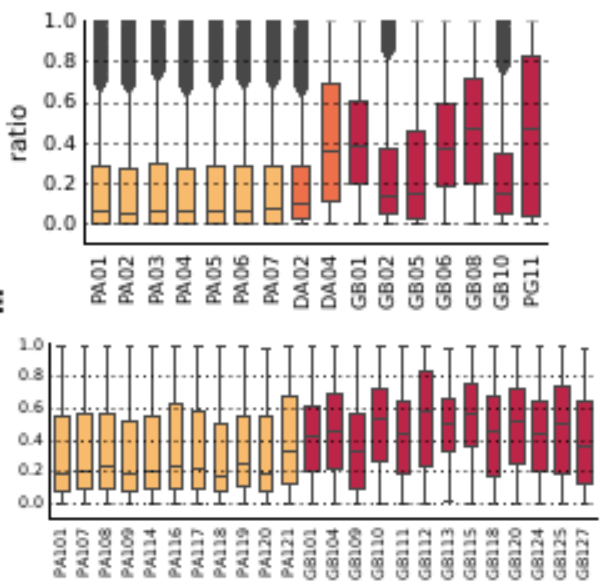

$\mathbf{F}$

Solute:sodium symporter activity

inorgaric cation transmembrane transport

cell differertiation

locomatory behavior

sodium ion transport

epithelium development

arimal organ developmert

sensory perception of sound

plasma menbrane region

synapse part

luteinizing hormone receptor activty

catecholamine binding

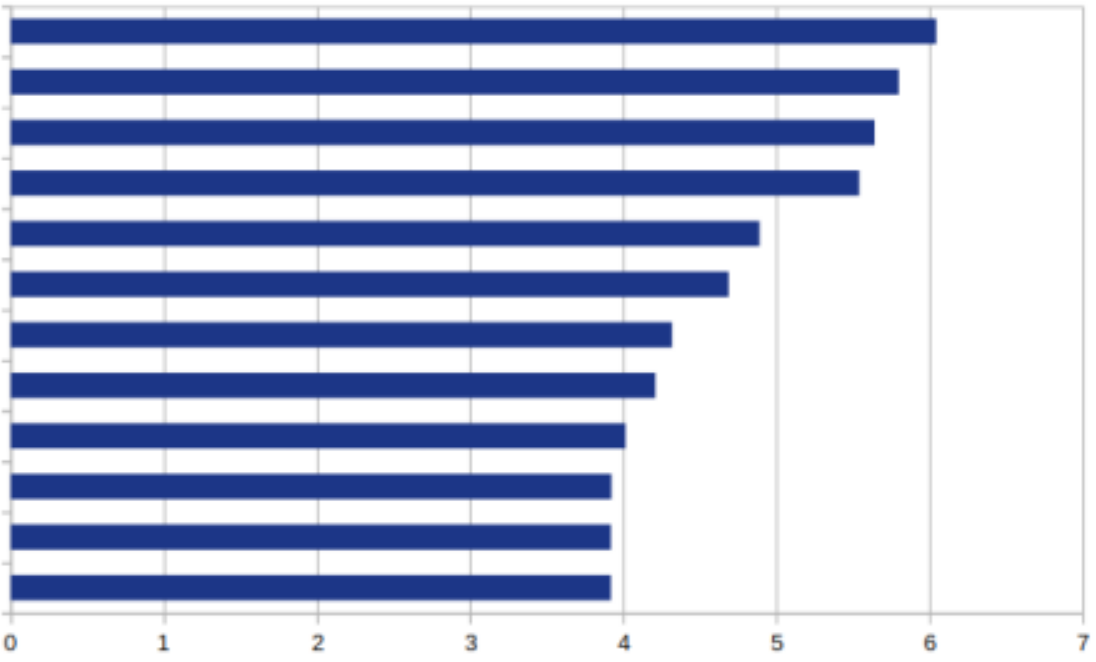


bioRxiv preprint doi: https://doi.org/10.1101/867861; this version posted March 20, 2020. The copyright holder for this preprint (which was availabBunder aCC-BY-NC-ND 4.0 International licene.

\section{9 putative}

enhancers

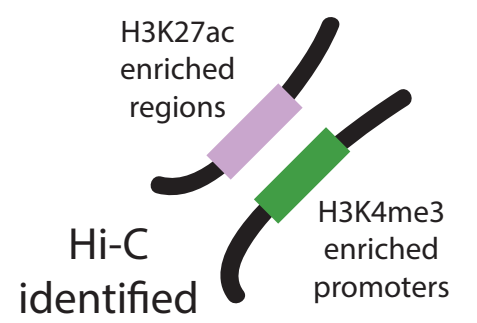

$17 \mathrm{DE}$

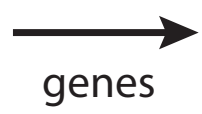

contacts

D

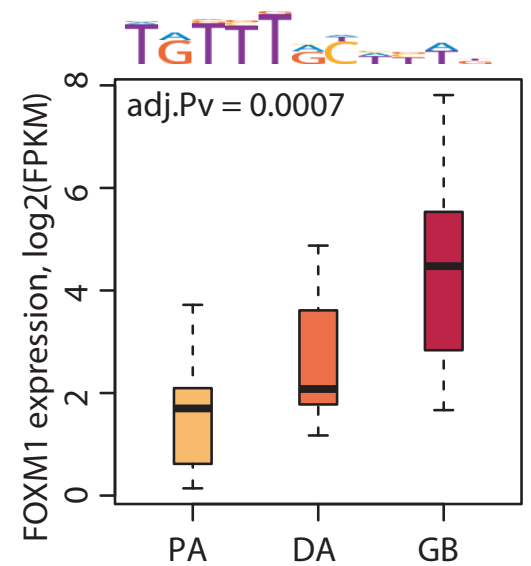

$\mathrm{G}$ Patients stratified by 85 gene
promoters

ANXA2R expression levels

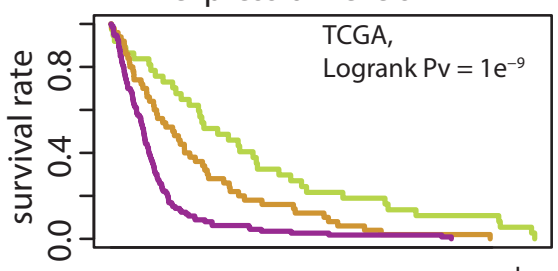

Patients stratified by low

FOXM1 expression levels high

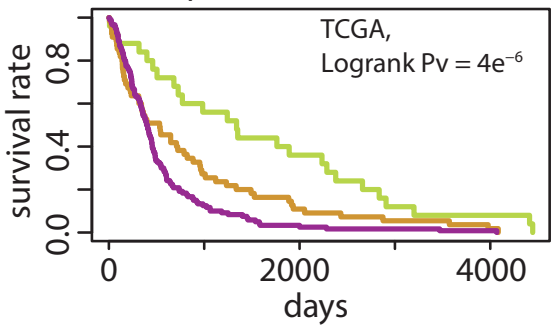

viewpoint

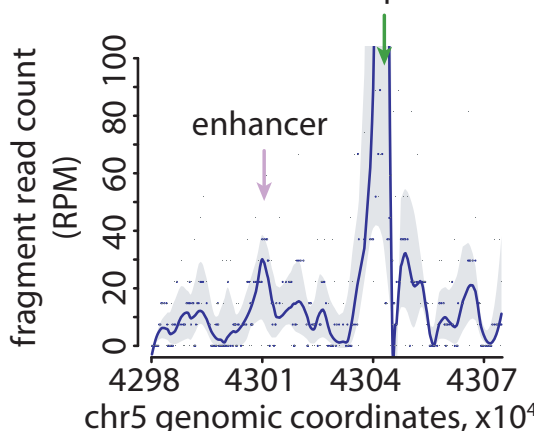

E

J
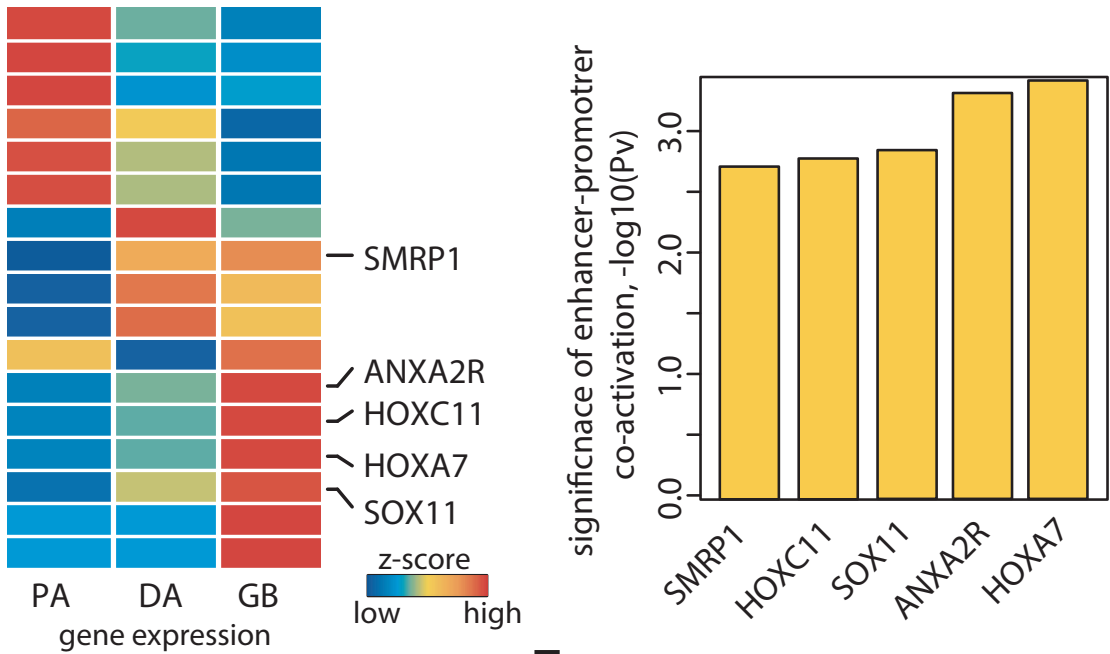

$\mathrm{F}$

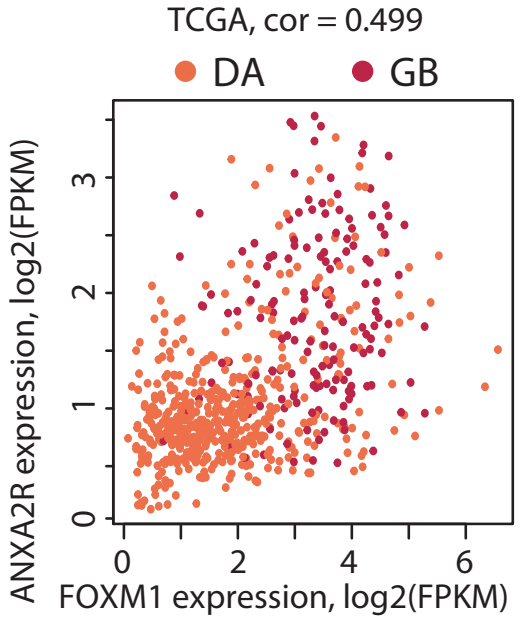

FOXM1 expression
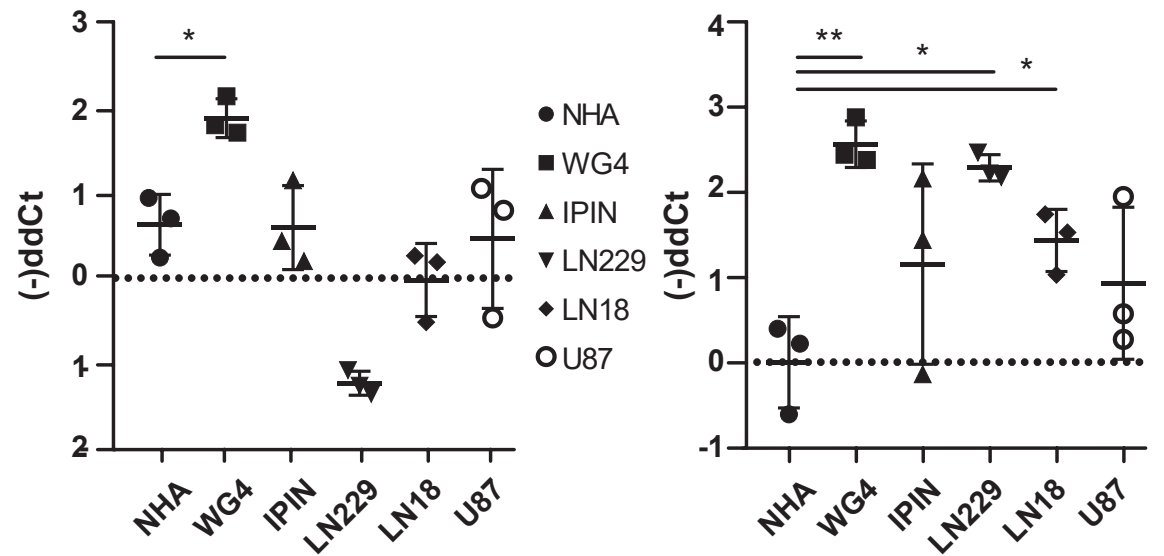

CCNB1
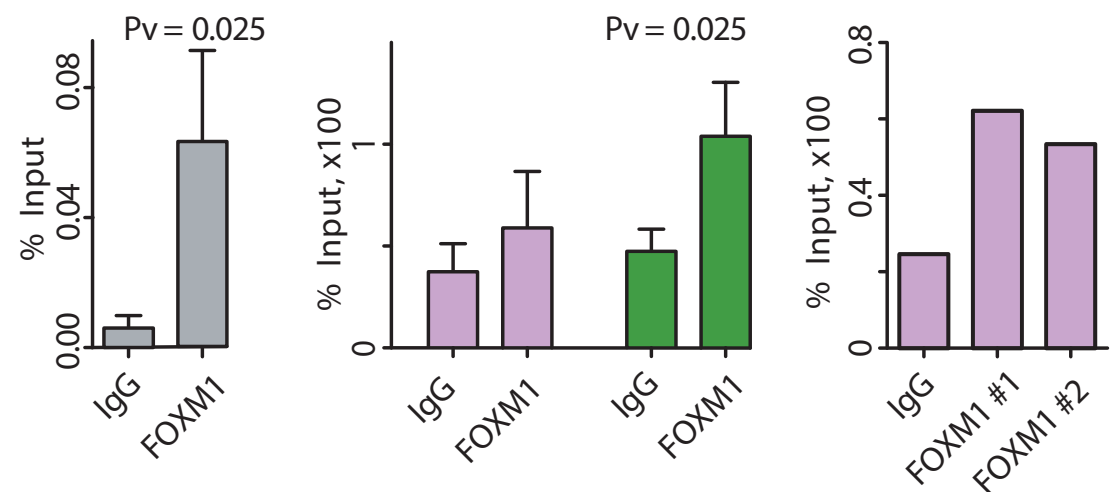

Figure 6 
KEY RESOURCES TABLE

\begin{tabular}{|c|c|c|}
\hline REAGENT or RESOURCE & SOURCE & IDENTIFIER \\
\hline \multicolumn{3}{|l|}{ Antibodies } \\
\hline Rabbit polyclonal anti-H3K4me3 & $\begin{array}{l}\text { Merck Milli- } \\
\text { pore }\end{array}$ & Cat. number 07-473, RRID:AB_1977252 \\
\hline Rabbit polyclonal anti-H3K27ac & Active Motif & Cat. number 39133, RRID:AB_2561016 \\
\hline Rabbit monoclonal anti-H3K27me3 & Abcam & Cat. number ab192985, RRID:AB_2650559 \\
\hline Rabbit polyclonal anti-FOXM1 & Diagenode & Cat. number C15410232-100 \\
\hline Rabbit Normal IgG Control Antibody, Unconjugated & $\begin{array}{l}\text { Cell Sig- } \\
\text { nalling } \\
\end{array}$ & Cat. number 2729, RRID:AB_1031062 \\
\hline \multicolumn{3}{|l|}{ Biological Samples } \\
\hline See Table S1 & This paper & NA \\
\hline \multicolumn{3}{|l|}{ Chemicals, Peptides, and Recombinant Proteins } \\
\hline $37 \%$ Formaldehyde & Sigma-Aldrich & CAS number $50-00-0$ \\
\hline A- and G-Sepharose beads & $\begin{array}{l}\text { Merck Milli- } \\
\text { pore }\end{array}$ & Cat. number $16-156 \& 16-266$ \\
\hline Adapters and USER enzyme & $\begin{array}{c}\text { New England } \\
\text { Biolabs }\end{array}$ & Cat. number E7335L \\
\hline Ampure Beads & $\begin{array}{c}\text { Beckman } \\
\text { Coulter }\end{array}$ & Cat. number A63880 \\
\hline DMEM & $\begin{array}{c}\text { ThermoFisher } \\
\text { Scientific }\end{array}$ & Ref 31885-023 \\
\hline DMEM/F-12, GlutaMAX'Tм & $\begin{array}{c}\text { ThermoFisher } \\
\text { Scientific }\end{array}$ & Ref 31966-021 \\
\hline DNA polymerase & EURx & Cat. number E2500-01 \\
\hline Dynabeads Protein A & $\begin{array}{c}\text { ThermoFisher } \\
\text { Scientific }\end{array}$ & Cat. number 10002D \\
\hline FBS & $\begin{array}{c}\text { ThermoFisher } \\
\text { Scientific }\end{array}$ & Cat. number 1056000 \\
\hline Mbol & NEB & Cat. number R0147M \\
\hline Proteinaise $\mathrm{K}$ & $\begin{array}{c}\text { Applied } \\
\text { Biosystems }\end{array}$ & Cat. number 4333793 \\
\hline RNase A & Invitrogen & Cat. number 12091-021 \\
\hline SimplySafe ${ }^{\mathrm{TM}}$ & EURx & Cat. number E4600-01 \\
\hline SYBR Green chemistry & $\begin{array}{c}\text { Applied } \\
\text { Biosystem by } \\
\text { Thermo } \\
\text { Fisher Scien- } \\
\text { tific } \\
\end{array}$ & Cat. Number 4385612 \\
\hline T4 DNA ligase & NEB & Cat. number M0202T \\
\hline TRI Reagent & Sigma-Aldrich & Product No.T9424 \\
\hline
\end{tabular}


Agilent DNA High Sensitivity Kit

Agilent RNA 6000 Nano Kit

ChIP-IT Express Immunoprecipitation Kit

EZ-96 DNA Methylation-Gold kit

Infinium HumanMethylation 450 BeadChip

KAPA Stranded mRNA Sample Preparation Kit

NEBNext Ultra Library Prep Kit for Illumina

Nextera DNA Library Preparation Kit

Syngen GEL/ PCR Mini Kit

QIAsymphony DNA Midi kit

QuantiFluor double stranded DNA System

RNeasy Mini kit

xGen Lockdown Probes and Reagents kit

Zymo DNA Clean and Concentrator 5
Agilent Tech-

nologies, Ltd.

Agilent Tech-

nologies, Ltd.

Active Motif

Zymo Re-

search

Illumina

Kapa Biosystems

Ilumina

Illumina

Syngen

Biotech

Qiagen

Promega

(Qiagen,

Hilden, Ger-

many)

IDT

Zymo Re-

search
Cat. number 5067-4626

Cat. number 5067-1511

Cat. number 53008

Cat. number D5007

Cat. IDs:WG-314-1003

Kit code KK8420 Roche cat no. 07962193001

Cat. number E7370S/L

Cat. number FC-121-1030

Cat. number SY201010

Cat. number 931255

Cat. number E2670

Cat. Number 74104

Cat. number 1072280

Cat. number D4003T

\section{Deposited Data}

DNA methylation data from human brain cerebellum and cortex

(Pidsley et

al., 2014)

(Lambert et al., 2013)

(Lambert et

al., 2013)
GSE61431

GSE44684

GSE44971

Gene expression data from pilocytic astrocytoma samples

\begin{tabular}{c|c}
\hline ATCC & \\
\hline ATCC & \\
\hline ATCC & \\
\hline Bozena & \\
Kaminska & Lab \\
\hline Bozena & \\
Kaminska & \\
Lab & \\
\hline
\end{tabular}

CRL-2611

CRL-2610

HTB-14

LN18

WG4

IPIN

LN229
LN18
U87
WG4
IPIN
H_mut_F
H_AGAAGCTATAA

GAPDH_ChIP_F TACTAGCGGTTTTACGGGCGCAC

GAPDH_ChIP_R TCGAACAGGAGGAGCAGAGAGCGA

HOXA7_ChIP_FAGATGCGGAAATTGGCCTCAG

HOXA7_ChIP_R TCCTACGACCAAAACATCCCC

\begin{tabular}{|c|c|}
\hline Oligo.pl & \\
\hline Oligo.pl & \\
\hline Oligo.pl & \\
\hline Oligo.pl & \\
\hline Oligo.pl & \\
\hline Oligo.pl & \\
\hline
\end{tabular}

NA

NA

NA

NA

NA

NA 


\begin{tabular}{|c|c|c|}
\hline $\begin{array}{c}\text { CCNB1_prom_ChIP_F CGCGATCGCCCTG- } \\
\text { GAAACGCA }\end{array}$ & Oligo.pl & NA \\
\hline $\begin{array}{c}\text { CCNB1_prom_ChIP_R CCCAGCAGAAACCAACAGC- } \\
\text { CGT }\end{array}$ & Oligo.pl & NA \\
\hline $\begin{array}{c}\text { ANXA2R_enh_ChIP_FATGGACAAACAAACCAA- } \\
\text { CAAACA }\end{array}$ & Oligo.pl & NA \\
\hline ANXA2R_enh_ChIP_R AGTGCAGTCCATGCAGGTTA & Oligo.pl & NA \\
\hline ANXA2R_prom_ChIP_F TGTGACCACCGATTCCACTG & Oligo.pl & NA \\
\hline ANXA2R_prom_ChIP_R ACAGACATTTGCTACGGGCA & Oligo.pl & NA \\
\hline ANXA2R_qPCR_F CAAGTACAGCGAAGCCCACT & Oligo.pl & NA \\
\hline ANXA2R_qPCR R CTGAGTCTGTCGGGTTCCTC & Oligo.pl & NA \\
\hline FOXM1_qPCR_F GCAGCGACAGGTTAAGGTTG & Oligo.pl & NA \\
\hline FOXM1_qPCR_R GTCATGCGCTTCCTCTCAGT & Oligo.pl & NA \\
\hline \multicolumn{3}{|l|}{ Software and Algorithms } \\
\hline Basic4CSeq Biocoductor package & walterC. 2019 & \\
\hline bedtools & $\begin{array}{l}\text { (Quinlan and } \\
\text { Hall, 2010) }\end{array}$ & \\
\hline bigWigAverageOverBed & & https://github.com/ENCODE-DCC/kentUtils \\
\hline Bowtie 2.2.6.2 & $\begin{array}{l}\text { (Langmead, } \\
\text { 2013) }\end{array}$ & \\
\hline BSMAP & $\begin{array}{c}\text { (Xi and Li, } \\
2009)\end{array}$ & \\
\hline CytoMeth & $\begin{array}{l}\text { Draminski et } \\
\text { al. in prep. }\end{array}$ & https://github.com/mdraminski/CytoMeth \\
\hline Dendextend R package & (Galili, 2015) & \\
\hline EDASeq & (Risso, 2013) & \\
\hline FastQC & & $\begin{array}{l}\text { https://www.bioinformatics.babraham.ac.uk/projects } \\
\text { fastqc/ }\end{array}$ \\
\hline FASTQ Trimmer & $\begin{array}{l}\text { (Blankenberg } \\
\text { et al., 2010) }\end{array}$ & \\
\hline FASTX & & http://hannonlab.cshl.edu/fastx_toolkit/ \\
\hline Filter FASTQ & $\begin{array}{l}\text { (Blankenberg } \\
\text { et al., 2010) }\end{array}$ & \\
\hline F-seq1.85 & $\begin{array}{l}\text { (Boyle et al., } \\
\text { 2008) }\end{array}$ & \\
\hline HiCEnterprise & & https://github.com/hansiu/HiCEnterprise \\
\hline HiTC Bioconductor package & $\begin{array}{l}\text { Servant N, } \\
2012 \\
\end{array}$ & \\
\hline MACS2.1 & $\begin{array}{l}\text { (Zhang et al., } \\
\text { 2008) }\end{array}$ & \\
\hline methylKit & $\begin{array}{l}\text { (Akalin et al., } \\
\text { 2012) }\end{array}$ & \\
\hline $\begin{array}{l}\text { PANTHER Overrepresentation Test (Released } \\
\text { 20171205) }\end{array}$ & $\begin{array}{l}\text { (Mi et al., } \\
2017)\end{array}$ & \\
\hline Picard & & http://broadinstitute.github.io/picard/ \\
\hline
\end{tabular}




\begin{tabular}{|c|c|c|}
\hline PWMEnrich R package & $\begin{array}{l}\text { (Stojnic and } \\
\text { Diez, 2018) }\end{array}$ & \\
\hline QuasR Bioconductor package & $\begin{array}{l}\text { (Gaidatzis D, } \\
\text { 2015) }\end{array}$ & \\
\hline samtools & $\begin{array}{l}\text { (Li et al., } \\
\text { 2009a) }\end{array}$ & \\
\hline Tophat2 & $\begin{array}{l}\text { (Kim et al., } \\
\text { 2013) }\end{array}$ & \\
\hline Trimmomatic & $\begin{array}{l}\text { (Bolger et al., } \\
\text { 2014) }\end{array}$ & \\
\hline \multicolumn{3}{|l|}{ Other } \\
\hline ENCODE Regulation 'Txn Factor' track data V3 & & $\frac{\text { http://hgdownload.soe.ucsc.edu/goldenPath/hg19/en }}{\text { codeDCC/wgEncodeRegTfbsClustered/ }}$ \\
\hline GO Ontology database Released 2018-06-01 & $\begin{array}{l}\text { (Ashburner et } \\
\text { al., 2000; } \\
\text { Carbon et al., } \\
\text { 2017) }\end{array}$ & \\
\hline HOCOMOCO v11 database & $\begin{array}{l}\text { (Kulakovskiy } \\
\text { et al., 2018) }\end{array}$ & \\
\hline PhastCons 100-Way scores & & $\frac{\text { http://hgdownload.cse.ucsc.edu/goldenPath/hg38/ }}{\text { phastCons100way/hg38.phastCons100way.bw }}$ \\
\hline
\end{tabular}

\section{EXPERIMENTAL MODEL AND SUBJECT DETAILS}

Human glioma samples

Freshly resected glioma specimens were acquired from several neurosurgery clinics: The Children's Memorial Health Institute, Public Central Clinical Hospital, Institute of Psychiatry and Neurology and Mazovian Brodno Hospital. The tissue collection protocol was approved by the Committees of Bioethics of the institutions listed above (protocol number \#14/KBE/2012, \#KBE/54/2016, \#3/2016). Each patient provided a written consent for use of tumor tissues; afterwards samples were anonymized. Details concerning age, gender, clinical diagnosis, tumor location and molecular test results are provided in the Table S1. Tumor samples were transported in DMEM/F-12 medium on ice and processed directly surgical resection. Procedures were halted at a first safe stop within 1-3 $\mathrm{h}$ after acquisition. Tumor specimens were transferred cold PBS, minced with sterile scissors or scalpel on a Petri dish kept on ice and subsequently homogenized by using chilled manual glass douncer. Then homogenized material was aliquoted to perform different methods.

\section{Human glioma cell lines}

Human malignant U-87 MG, LN18 and LN229 glioblastoma cells were purchased from American Type Culture Collection (ATCC). WG4 and IPIN are primary glioma cell lines developed from GBM patient surgical samples as described before (Ciechomska et al., 2016).

\section{Method Details}

Established glioma cell lines were cultured in Dulbecco's modified Eagle medium (DMEM) supplemented with $10 \%$ fetal bovine serum (ThermoFisher Scientific) and 100 units $/ \mathrm{mL}$ of penicillin and $100 \mu \mathrm{g} / \mathrm{mL}$ of streptomycin. Primary glioma cell lines were cultured in DMEM/Nutrient Mixture F- 
12, GlutaMAX ${ }^{\mathrm{TM}}$ medium (DMEM/F-12, GlutaMAX ${ }^{\mathrm{TM}}$ ) supplemented with $10 \%$ fetal bovine serum (ThermoFisher Scientific) and antibiotics (100 U/mL penicillin, $100 \mu \mathrm{g} / \mathrm{mL}$ streptomycin).

\section{Nucleic acids extraction from glioma samples}

Total RNA and DNA were isolated using Tri-Reagent extraction (Sigma-Aldrich, Munich, Germany) starting from 50-100 mg of tissues(depending on the initial specimen size). RNA quality and yield were verified by Bioanalyzer 2100 (Agilent Technologies, Santa Clara, CA) using a RNA 6000 Nano Kit (Agilent Technologies, Ltd.) and NanoDrop 2000 (Thermo Scientific, NanoDrop products, Wilmington, USA). DNA purity was estimated using NanoDrop 2000 (Thermo Scientific, NanoDrop products, Wilmington, USA).

\section{Analysis of IDH1/2 mutations}

To check IDH1/2 status, $20 \mathrm{ng}$ of genomic DNA was amplified by polymerase chain reaction (PCR) with specific primers amplifying the exon 6,in which common IDH mutations occur. PCR reaction consisted standard buffer, forward primer 5'-GGATGCTGCAGAAGCTATAA-3', reverse primer 5'CATGCAAAATCACATTATTGCC-3'. s and DNA polymerase (EURx) in a total volume of $25 \mathrm{~mL}$, and followed denaturation at $95^{\circ} \mathrm{C}$ for $30 \mathrm{~s}$, annealing at $54^{\circ} \mathrm{C}$ for $30 \mathrm{~s}$ and extension at $72^{\circ} \mathrm{C}$ for $50 \mathrm{~s}$ for 35 cycles. PCR products were separated on $1 \%$ agarose gel as 230 bp fragments, and visualized by SimplySafe ${ }^{\text {TM }}$ (EURx) stainingSubsequently, PCR products were purified using Syngen GEL/ PCR Mini Kit (Syngen Biotech) and a mutational status of $I D H$ gene in surgical glioma specimens was determined by Sanger sequencing.

\section{RNA sequencing}

Strand-specific polyA enriched RNA libraries were prepared using the KAPA Stranded mRNA Sample Preparation Kit according to the manufacturer's protocol (Kapa Biosystems, MA, USA). Briefly, mRNA molecules were enriched from 500ng of total RNA using poly-T oligo-attached magnetic beads (Kapa Biosystems, MA, USA). Obtained mRNA was fragmented and the first-strand cDNA was synthesized using a reverse transcriptase. Second CDNA synthesis was performed to generate double-stranded cDNA (dsDNA). Adenosines were added to the $3^{\prime}$ ends of dsDNA and adapters were ligated (adapters from NEB, Ipswich, MA, USA). Following the adapter ligation, uracil in a loop structure of adapter was digested by USER enzyme from NEB (Ipswich, MA, USA). Adapters containing DNA fragments were amplified by PCR using NEB starters (Ipswich MA, USA). Library evaluation was done with Agilent 2100 Bioanalyzer using the Agilent DNA High Sensitivity chip (Agilent Technologies, Ltd.) Mean library size was 300bp. Libraries were quantified using a Quantus fluorometer and QuantiFluor double stranded DNA System (Promega). Libraries were run in the rapid run flow cell and were paired-end sequenced (2x76bp) on HiSeq 1500 (Illumina, San Diego, CA 92122 USA).

\section{ATAC-sequencing}

In order to obtain single cell suspension, preliminarily disrupted tumor sample aliquots corresponding to 50-100 mg of tissue were passed through a syringe needle around 50 times. Mechanical homogenization was followed by centrifugation for $5 \mathrm{~min}$ at $4^{\circ} \mathrm{C}$ at $2400 \mathrm{~g}$. Each pellet was resuspended in 10 $\mathrm{ml}$ of cold lysis buffer L1 (50 mM HEPES KOH, pH7.5, $140 \mathrm{mM} \mathrm{NaCl}, 1 \mathrm{mM}$ EDTA pH 8.0, 10\% glycerol, $5 \%$ NP-40, $0.25 \%$ Triton X-100, containing proteinase inhibitor cocktail) and incubated at $4^{\circ} \mathrm{C}$ for $20 \mathrm{~min}$ on a rocking shaker. Then mechanical forcing of tissue disruption was repeated, residual de- 
bris were precleared by filtration through an $80-\mu \mathrm{m}$ nylon mesh filter and eventually lysis buffer was replaced with PBS. Cell suspension was visually controlled under the microscope. Cells were counted automatically with NucleoCounter NC-100 and 50,000 cells were subsequently lysed as previously described (Buenrostro et al., 2013). Then transposition reaction was performed using Nextera DNA Library Preparation kit by Illumina, accordingly to the Buenrostro protocol. Reactions were cleaned up with Zymo DNA Clean and Concentrator 5 columns. The remainder of the ATAC-seq library preparation was performed as described previously (Buenrostro et al., 2013). Finally, ATAC-seq libraries were visualized on Bioanalyzer 2100 (Agilent Technologies, Santa Clara, CA) and generated chromatograms were used to estimate DNA concentration. Libraries were run in the rapid run flow cell and were paired-end sequenced (2x76bp) on HiSeq 1500 (Illumina, San Diego, CA 92122 USA).

\section{Chromatin immunoprecipitation (ChIP) on tissue samples}

Cell suspensions corresponding to $100-400 \mathrm{mg}$ of tumor tissue were aliquoted and spuned down at $1200 \mathrm{rpm}$ for $10 \mathrm{~min}$ at $4^{\circ} \mathrm{C}$ in a swing bucket centrifuge (Eppendorf Centrifuge 5810R). Pellets were crosslinked in a crosslinking buffer containing $100 \mathrm{mM} \mathrm{NaCl}, 50 \mathrm{mM}$ HEPES, $1 \mathrm{mM}$ EDTA, $0.5 \mathrm{mM}$ EGTA and supplemented with 1\% formaldehyde (Sigma-Aldrich) for 15 minutes at room temperature on a rocking shaker. Fixation was stopped by incubation with $0.125 \mathrm{M}$ glycine for $5 \mathrm{~min}$ at RT. In order to wash away the excess of formaldehyde, fixed material was twice centrifuged at $1400 \mathrm{~g}$ for 10 min at $4^{\circ} \mathrm{C}$ and then pellets were resuspended in cold PBS supplemented with a protease inhibitor cocktail (Roche). Eventually, pellets were stored at $-80^{\circ} \mathrm{C}$ before starting further procedure. Thawed on ice, pellets were resuspended in $5 \mathrm{ml}$ of cold PBS supplemented with proteinase inhibitor cocktail and further homogenized with insulin syringes prior to obtain single cell suspension (if needed fixed tissue was additionally processed with mechanical homogenizer [PRO Scientific, PRO200] before using syringes). Homogenization step was followed by centrifugation for 5 min at $4{ }^{\circ} \mathrm{C}$ at $2400 \mathrm{~g}$. Each pellet was resuspended in $10 \mathrm{ml}$ of cold lysis buffer L1 (50 mM HEPES KOH, pH7.5, $140 \mathrm{mM} \mathrm{NaCl}, 1$ $\mathrm{mM}$ EDTA pH 8.0, 10\% glycerol, 5\% NP-40, 0.25\% Triton X-100, containing a proteinase inhibitor cocktail) and incubated at $4^{\circ} \mathrm{C}$ for $20 \mathrm{~min}$ on a rocking shaker. Subsequently, ice-cold manual glass douncer was used to release the nuclei and the remaining material was collected by centrifugation for 10 min at $4^{\circ} \mathrm{C}$ at $1700 \mathrm{~g}$. L1 buffer was changed for $10 \mathrm{ml}$ of warmed lysis buffer $\mathrm{L} 2(200 \mathrm{mM} \mathrm{NaCl}, 1$ mM EDTA pH 8.0, $0.5 \mathrm{mM}$ EGTA pH 8.0, $10 \mathrm{mM}$ Tris pH 8.0, containing proteinase inhibitor cocktail) and incubated at room temperature for $20 \mathrm{~min}$ on a rocking shaker, then manual glass homogenizer was used once again. This step was followed by centrifugation for 5 min at $4{ }^{\circ} \mathrm{C}$ at $1700 \mathrm{~g}$. Collected material was resuspended in $0.5 \mathrm{ml}$ of L3 buffer (1 mM EDTA pH 8.0, 0.5 mM EGTA pH 8.0, $10 \mathrm{mM}$ Tris $\mathrm{pH} 8.0,100 \mathrm{mM} \mathrm{NaCl}, 0.1 \% \mathrm{Na}$-deoxycholate, $0.17 \mathrm{mM} \mathrm{N}$-lauroyl sarcosine, containing protease inhibitors) and sonicated using a Bioruptor Plus Sonicator (Diagenode) for $3 \times 15$ cycles (30 ON: 30 OFF) set on HIGH conditions. Lengths of chromatin fragments (200-500 bp) were evaluated on agarose gels. Prior to do gel electrophoresis, batches of $10 \mathrm{ul}$ of chromatin were collected by centrifugation at 14, $000 \mathrm{rpm}$ (Eppendorf Centrifuge 5430) and after adding 1xTE buffer up to $300 \mathrm{ul}$, samples were incubated overnight at $65^{\circ} \mathrm{C}$ with occasional shaking. After reversal of crosslinking, samples were treated with RNase I at $0.1 \mathrm{mg} / \mathrm{ml}$ for $30 \mathrm{~min}$ at $37^{\circ} \mathrm{C}$, and then treated with proteinase $\mathrm{K}$ (final concentration $0.4 \mathrm{mg} / \mathrm{ml}$ ) for $1.5 \mathrm{~h}$ at $55^{\circ} \mathrm{C}$. The DNA was purified by phenol-chloroform extraction followed by ethanol precipitation, and recovered in $20 \mu \mathrm{l}$ of water. Additionally, the efficiency of chromatin isolation was evaluated with the measurement on Quantus Fluorometer (Promega). 
DNA-protein complexes were immunoprecipitated with $5 \mu \mathrm{g}$ of antibody against H3K4me3 (cat. Number 07-473, Merck Millipore), H3K27ac (cat. Number 39133, Active Motif) and H3K27me3 (cat. number ab192985, Abcam). In order to estimate specificity and purity of ChIP reaction, immunoprecipitation with normal IgG was performed simultaneously (cat. Number 2729S, Cell Signalling). Generally, $30 \mu \mathrm{g}$ of fragmented chromatin was added per ChIP reaction. Depending on antibody type, different ChIP protocols were applied. Anti-H3K4me3 immunoprecipitation and input sample processing were carried out accordingly to the protocol provided with ChIP-IT Express Immunoprecipitation Kit (Cat. Number 53008, Active Motif) with some modifications: i) the volume of IP reaction was equal 0.5 or 1 $\mathrm{ml}$, depending on chromatin concentration in L3 buffer, ii) each washing step of magnetic beads was repeated twice, iii) elution lasted $30 \mathrm{~min}$. Decrosslink and DNA purification was performed as for sonication test. Anti-H3K27ac and anti-H3K27me3 immunoprecipitation was performed accordingly to the RoadmapEpigenomics protocol for ChIP-seq on tissues (http://www.roadmapepigenomics.orgl protocols/type/experimental//). Briefly, $30 \mu \mathrm{g}$ of pre-cleared chromatin was incubated for $4 \mathrm{~h}$ at $4^{\circ} \mathrm{C}$ with A- and G-Sepharose beads Cat. number 16-156 \& 16-266, Millipore) mix with pre-bound appropriate antibodies. The beads were washed once with RIPA-150 buffer, twice with RIPA-500, twice with RIPA-LiCl and twice with TE buffer, and then bound chromatin was eluted with freshly made elution buffer. After decrosslink and DNA purification, acquired DNA was measured with Quantus fluorometer using Agilent 2100 Bioanalyzer and High Sensitivity DNA kit (Agilent Technologies, Ltd). Before DNA library preparation, an immunoprecipitated material was evaluated by GPCR with specific primers designed to amplify the GAPDH promoter region (active chromatin marks) or the HOXA7 gene body region (repressive chromatin mark) using SYBR Green chemistry (Cat. Number 4385612, Applied Biosystem by Thermo Fisher Scientific) and QuantStudio 12K Flex Real-Time PCR System (data not shown).

\section{ChIP sequencing}

DNA libraries for chromatin immunoprecipitation with respective antibodies were prepared using QIAseq Ultra Low Input Library Kit (QIAGEN, Hilden, Germany). Briefly, DNA was end-repaired, adenosines were added to the $3^{\prime}$ ends of dsDNA and adapters were ligated (adapters from NEB, Ipswich, MA, USA). Following the adapter ligation, uracil was digested by USER enzyme from NEB (Ipswich, MA, USA) in a loop structure of adapter. Adapters containing DNA fragments were amplified by PCR using NEB starters (Ipswich MA, USA). Library quality evaluation was done with Agilent 2100 Bioanalyzer using the Agilent DNA High Sensitivity chip (Agilent Technologies, Ltd.) Quantification and quality evaluation of obtained samples were done using Nanodrop spectrophotometer (Thermo Scientific, NanoDrop products, Wilmington, USA), Quantus fluorometer (Promega Corporation, Madison, USA) and 2100 Bioanalyzer (Agilent Technologies, Santa Clara, USA). Mean library size was $300 \mathrm{bp}$. Libraries were run in the rapid run flow cell and were single-end sequenced (65bp) on HiSeq 1500 (Illumina, San Diego, CA 92122 USA).

\section{ChIP-qPCR on cultured glioma cells}

Primary glioma WG4 cell cultures were harvested at $90 \%$ confluency using standard trypsinization protocol and $1 \times 10^{7}$ cells were collected by centrifugation (1200 rpm, $10 \mathrm{~min}, 4^{\circ} \mathrm{C}$, Eppendorf Centrifuge 5810R), and fixed in 1\% formaldehyde (ThermoFisher Scientific) diluted in PBS for 15 minutes at room temperature on a rocking shaker. Formaldehyde was quenched by adding $0.125 \mathrm{M}$ glycine and incubating samples for 5 min at RT. Fixed cells were centrifuged at $1400 \mathrm{~g}$ for 10 min at $4{ }^{\circ} \mathrm{C}$ and 
washed twice with PBS as described. Cell pellets were resuspended in $10 \mathrm{ml}$ of cold L1 buffer and incubated at $4^{\circ} \mathrm{C}$ for $20 \mathrm{~min}$ on a rocking shaker to force cell swelling. Subsequently, ice-cold manual glass douncer was used to aid nuclei release which were collected by centrifugation $\left(10 \mathrm{~min}, 4^{\circ} \mathrm{C}\right.$, $1200 \mathrm{rpm}$, Eppendorf Centrifuge 5810R) in order to reduce sample volume. After centrifugation, cell pellets were resuspended in $1 \mathrm{ml}$ of buffer, transferred to Eppendorf tubes and pushed through insulin syringe needle several times. Nuclei were centrifugated $\left(10 \mathrm{~min}\right.$ at $4^{\circ} \mathrm{C}$ at $1200 \mathrm{rpm}$, Eppendorf Centrifuge 5430), and reconstituted in $50 \mu \mathrm{l} \mathrm{SDS}$ lysis buffer (1\% SDS, $10 \mathrm{mM}$ EDTA, $50 \mathrm{mM}$ Tris- $\mathrm{HCl}$, $\mathrm{pH}=8.0$ ) and incubated on ice for $30 \mathrm{~min}$. Nuclei release was controlled under the microscope and $300 \mu \mathrm{l}$ of $1 \mathrm{x}$ TE were added to dilute SDS. Chromatin fragmentation was performed in Eppendorf tubes using Bioruptor Plus Sonicator (Diagenode) with the following program: $3 \times 13$ cycles (30 ON: 30 OFF), HIGH conditions. Sonication efficacy was examined using agarose gel electrophoresis as described above in ChIP on tissue samples section. Chromatin isolated from $1 \times 10^{7}$ cells was used per one immunoprecipitation reaction either with $5 \mu \mathrm{g}$ FOXM1 (cat. no. C15410232-100, Diagenode) or normal rabbit IgG antibody (cat. no. PP64B, Merck Millipore). Immunoprecipitated complexes were captured on Dynabeads Protein A (cat. no. 10002D, ThermoFisher Scientific) accordingly to the RoadmapEpigenomics protocol mentioned before (Dynabeads part) with modified elution step performed as follows: $125 \mu \mathrm{l}$ elution buffer was added to washed beads and resuspended beads were kept in $65^{\circ} \mathrm{C}$ for $10 \mathrm{~min}$, this elution step was repeated and eventually eluate was pooled. Decrosslink, RNase, proteinase $\mathrm{K}$ treatments and DNA purification was performed as described in the ChIP on tissue samples section. Real-time PCR amplifications of the studied ANXA2R regulatory regions were performed applying SYBR Green chemistry (Cat. Number 4385612, Applied Biosystem by Thermo Fisher Scientific) on QuantStudio 12K Flex Real-Time PCR System device using primers indicated in Key Sources Table. The CCNB1 gene promoter served as a positive control for FOXM1 binding. Results were calculated as $\%$ of input, mean $\pm S D(n=3)$ and compared to IgG values. T-test was used for evaluation fo sgnificance.

\section{DNA methylation sequencing}

DNA samples) were bisulfite-converted using EZ DNA Methylation-Lightning Kit (Zymo Research, Irvine, CA, USA). Probes from SeqCap Epi CpGiant Enrichment Kit (Hoffmann-La Roche, Basel, Switzerland) were used to enrich each Bisulfite-Converted Sample Library in the predetermined various genome regions of $>80.4 \mathrm{Mb}$ capture size comprising $>5.6$ million of $\mathrm{CpG}$ sites on both DNA strands. The libraries were prepared according to the Hoffmann-La Roche's "NimbleGen SeqCap Epi Library Workshop Protocol, v1.0" and "SeqCap Epi Enrichment System User's Guide, v1.2". Briefly, the concentration of genomic DNA was measured using a Quantus fluorometer with QuantiFluor dsDNA System (Promega, Madison, WI, USA) and $1 \mu \mathrm{gg}$ of input DNA together with $165 \mathrm{pg}$ of Bisulfite-Conversion Control (viral unmethylated gDNA; SeqCap Epi Accessory Kit; Hoffmann-La Roche) were fragmented using Focused-ultrasonicator Covaris M220 (Covaris, Inc., Woburn, MA, USA) to an average size of $200 \pm 20 \mathrm{bp}$. DNA fragments were checked using the High Sensitivity DNA Kit on a 2100 Bioanalyzer (Agilent Technologies, Inc., Santa Clara, CA, USA). Next, DNA fragments were "End-Repaired", "A-Tailing" was performed and Index Adapters ligated using KAPA LTP Library Preparation Kit (KAPA Biosystems, Wilmington, USA), SeqCap Adapter Kit A and B (Hoffmann-La Roche) and DNA purification beads (Agencourt AMPure XP Beads; SeqCap EZ Pure Capture Bead Kit; Hoffmann-La Roche). Then, DNA fragments, enlarged by adapters, were size selected Agencourt AMPure XP Beads (SeqCap EZ Pure Capture Bead Kit) using Solid Phase Reversible Immobilization technology to discard DNA fragments larger than $\sim 450$ and smaller than 
$\sim 250$ bp. Next, the libraries were bisulfite-converted using EZ DNA Methylation-Lightning Kit (Zymo Research) and amplified by Pre-Capture Ligation Mediated PCR (LM-PCR). After cleaning on Agencourt AMPure XP Beads (SeqCap EZ Pure Capture Bead Kit), quality and concentrations of Amplified Bisulfite-Converted Sample Libraries were determined using NanoDrop spectrophotometer (Thermo Fisher Scientific, Waltham, MA, USA) and Quantus with QuantiFluor dsDNA System (Promega), respectively. Also a size of DNA fragments was analyzed using the High Sensitivity DNA Kit on a 2100 Bioanalyzer (Agilent Technologies, Inc.). Next, $1 \mu \mathrm{g}$ of each Amplified BisulfiteConverted Sample Library was hybridized $\left(47^{\circ} \mathrm{C}, 67 \pm 2 \mathrm{~h}\right.$ ) with probes from SeqCap Epi CpGiant Enrichment Kit (Hoffmann-La Roche), bound to the Capture Beads (SeqCap EZ Pure Capture Bead Kit; Hoffmann-La Roche) and sequentially washed out of contamination and unspecific DNA in buffers of SeqCap Hybridization and Wash Kit (Hoffmann-La Roche). Finally, the Captured BisulfiteConverted Sample Libraries were amplified in Post-Capture LM-PCR, cleaned up using Agencourt AMPure XP Beads (SeqCap EZ Pure Capture Bead Kit) and the Amplified Captured BisulfiteConverted Sample Libraries were submitted to the last quality check where the quality and the concentrations of the final libraries were determined using NanoDrop (Thermo Fisher Scientific) and Quantus with QuantiFluor dsDNA System (Promega), respectively. A size of the obtained DNA fragments was also analyzed using the High Sensitivity DNA Kit on a 2100 Bioanalyzer (Agilent Technologies, Inc.). Libraries were run in the rapid run flow cell and were paired-end sequenced (2x76bp) on HiSeq 1500 (Illumina, San Diego, CA 92122 USA).

\section{DNA methylation bead chip analysis}

Genomic DNA was extracted using the QIAsymphony DNA Midi kit (Qiagen, Crawley, UK). Bisulphite conversion of $500 \mathrm{ng}$ of each sample was performed using the EZ-96 DNA Methylation-Gold kit (Zymo Research, Orange, CA, USA). Bisulphite-converted DNA was used for hybridization on the Infinium HumanMethylation 450 BeadChip (Bibikova et al., 2011)[according to manufacturer's instructions. Illumina GenomeStudio software was used to extract the raw signal intensities of each probe (without background correction or normalization).

\section{Quantitative PCR (qRT-PCR)}

Total RNA was extracted from glioma cells using the RNeasy Mini kit (Qiagen, Hilden, Germany) and purified using RNeasy columns accordingly to the manufacturer's instructions. cDNA was synthetized by extension of oligo(dT) primers with SuperScript III Reverse Transcriptase (Invitrogen, USA). Real-time PCR was performed applying SYBR Green chemistry (Cat. Number 4385612, Applied Biosystem by Thermo Fisher Scientific) on QuantStudio 12K Flex Real-Time PCR System device using primers indicated in Key Sources Table. Amplified product was normalized to the endogenous expression of GAPDH and represented as minus delta delta $\mathrm{Ct}$ values. $\mathrm{P}$ values were considered significant when ${ }^{*} \mathrm{P}<0.05$ and ${ }^{* *} \mathrm{P}<0.01$ (t-test).

\section{Capture-C assay}

WG4 cells were cultured, harvested and crosslinked following the same protocol as described in the ChIP-qPCR section. Cell pellet was resuspended in $500 \mu \mathrm{L}$ of freshly prepared ice-cold lysis buffer (10 mM Tris- $\mathrm{HCl}, \mathrm{pH} 8,10 \mathrm{mM} \mathrm{NaCl}, 0.2 \%$ Igepal NP-40 (Sigma), 1x protease inhibitor cocktail (Roche)), and incubated on ice for $30 \mathrm{~min}$ and followed by centrifugation to pellet nuclei (5 min, 1200 rpm, $4{ }^{\circ} \mathrm{C}$, Eppendorf Centrifuge 5430). Cells were washed once with 300 ul 1x NEB2 buffer, and nuclei were extracted by $40 \mathrm{~min}$ incubation at $37^{\circ} \mathrm{C}$ in $190 \mu \mathrm{l} 0.5 \%$ SDS $1 \times \mathrm{xNEB} 2$ buffer. In order to 
quench SDS, samples were transferred on ice and $400 \mu \mathrm{L}$ of 1 X NEBuffer2 and $120 \mu \mathrm{L}$ of $10 \%$ Triton $\mathrm{X}-100$ were added (in the mentioned order), and kept at $37^{\circ} \mathrm{C}$ for $15 \mathrm{~min}$. After centrifugation and washing with $1 \mathrm{X}$ NEBuffer2, the digestion reaction with $400 \mathrm{U}$ of Mbol was performed overnight in $37^{\circ} \mathrm{C}$ (300 $\mu$ l final volume) in termomixer with shake $800 \mathrm{RPM}$. The next day, nuclei were pelleted again, reconstituted in $200 \mu \mathrm{l}$ 1XNEB2 buffer and additional $200 \mathrm{U}$ of Mbol were added for two more hours before heat inactivation $\left(65^{\circ} \mathrm{C}, 15 \mathrm{~min}\right)$. After centrifugation, supernatant was discarded and obtained pellets were resuspended in $1.2 \mathrm{ml}$ of ligation mix consisting of 1x T4 DNA Ligase buffer, $1 \%$ Triton X-100 and $0.1 \mathrm{mg} / \mathrm{ml} \mathrm{BSA}$. Eventually $5 \mu \mathrm{L}$ of $2000 \mathrm{U} / \mu \mathrm{L}$ T4 DNA Ligase (NEB) was added and incubated at $16^{\circ} \mathrm{C}$ for $5 \mathrm{~h}$ to perform slowligation. Then samples wers centrifuged and nuclei were resuspended in $200 \mu \mathrm{l} 1 \mathrm{X}$ NEBuffer2. Decrosslink was performed by overnight incubation at $65^{\circ} \mathrm{C}$. The next day, we performed RNAse $A\left(10 \mu \mathrm{L}\right.$ of $\left.10 \mathrm{mg} / \mathrm{mL}, 30 \mathrm{~min}, 37^{\circ} \mathrm{C}\right)$ and Proteinase $\mathrm{K}$ treatment $\left(20 \mu \mathrm{L}\right.$ of $20 \mathrm{mg} / \mathrm{mL}, 1.5 \mathrm{~h}, 55^{\circ} \mathrm{C}$ ) followed by standard fenol:chloroform extraction to purify DNA. At the same time non-digested and non-ligated controls were processed, as $10 \mu \mathrm{l}$ of reaction at the crucial steps were taken to check digestion and ligation efficiency by running DNA on $0.8 \%$ agarose gel. Properly digested and re-ligated samples were subsequently used for library preparation. First, DNA was fragmented using Diagenode Bioraptor Pico device (cat. No. B01060010). Aliquots of 100 $\mu \mathrm{l}$ DNA $(10 \mu \mathrm{g} / \mu \mathrm{l})$ were distributed to 6 Eppendorf $1.5 \mathrm{ml}$ tubes, , and the following conditions: 20 "ON/60"OFF, 10 cycles were applied program. Sonication efficiency was tested by loading $50 \mathrm{ng}$ DNA on the $1.2 \%$ agarose gel. After AMPure beads (Beckman Coulter) purification $1 \mu \mathrm{mg}$ of fragmented DNAs were used for sequencing library generation using NEBNext Ultra Library Prep Kit for Illumina (cat. No. E7370S/L) following a manufacturer's protocol. The library was hybridized to probes capturing the $A N X A 2 R$ promoter fragment. Probe design excluded repeating elements present in the $A N X A 2 R$ promoter region. In order to capture both strands of a selected restriction fragment, biotinylated olignucleotides complementary to $5^{\prime}$ end of each strand were used (IDT). Lyophilized DNA oligonucleotides were reconstituted to a concentration of $2.9 \mu \mathrm{M}$, mixed and diluted in equimolar quantities to final $2.9 \mathrm{nM}$ concentration. Subsequently, Hybridization Capture of DNA libraries using xGen Lockdown Probes and Reagents kit (IDT) was used following a manufacturer's instruction with an exception of using NEBNext Ultra Library Prep Kit for Illumina (cat. No. E7370S/L) instead of recommended libarary preparation kit. Final library was sequenced using MiSeq MICRO 2x151 bps 300 cyles PE Cycle Paired-end sequencing (4M reads).

Quality of fastq files was assessed using FastQC tool (https://www.bioinformatics.babraham.ac.uk/projects/fastqc/) and trimmed with FASTQ Trimmer from $3^{\prime}$ end to reach a read length of 81 base pairs. Reads were aligned to human hg38 genome using qAlign function from Bioconductor QuasR package with maxHits parameter set to 1 to obtain uniquely mapped reads. At this point fastq files were treated as files obtained out of single-end sequencing experiments. Generated BAM files were sorted with Picard, and subsequently same tool (MarkDuplicates option) was used to remove PCR duplicates. We calculated the number of reads mapped to Mbol restriction fragments qAlign function (QuasR package). Briefly, new qProject object was created out of sorted and deduplicated BAM files, a list of restriction fragments for chromosome 5 generated with getRestrictionFragmentsPerChromosome function (HiTC package) were transformed into query object and mask parameter was set to exclude captured restriction fragment applying vmatchPattern function from Biostrings package. At this point read counts for each file were merged and normalized to „per million reads”. Results were visualized with visualizeViewpoint function from Basic4Cseq package.

\section{Data analysis}


Fastq preprocessing. The quality of raw fastq files was assessed using FastQC software (https://www.bioinformatics.babraham.ac.uk/projects/fastqc/), and based on the results the following parameters of preprocessing were chosen: i) for RNA-seq reads were trimmed with FASTQ Trimmer (Blankenberg et al., 2010) (offset from 5' end: 11, offset from 3 ' end: 3 ), overrepresented sequences were removed using clip tool from FASTX package (http://hannonlab.cshl.edu/fastx_toolkit/) and reads which pair mate was filtered out were removed; ii) for ChIP-seq reads were trimmed with FASTQ Trimmer (offset from 5' end: 5 for samples GB07, GB10, 3 for the rest; offset from 3' end: 3), Filter FASTQ (Blankenberg et al., 2010) was used to keep only reads with quality above specified threshold (min quality 10, max number of bases below this quality: 6), for patients DA05 and GB03, additionally we run Trimmomatic (Bolger et al., 2014) with option "sliding window 4", finally the reads were filtered by length using Filter FASTQ (min. length: 20); iii) for ATAC-seq reads were trimmed with FASTQ Trimmer (offset from 5' end: 14; offset from 3' end: 3), Filter FASTQ was used to keep only reads with quality above specified threshold (min quality 10, max number of bases below this quality: 5), the reads were filtered by length using Filter FASTQ (min. length: 20) and reads which pair mate was filtered out were removed. All fastq preprocessing steps were performed using Galaxy wrappers (Afgan et al., 2016).

Mapping, peak calling, read counts. ChIP-seq and ATAC-seq reads were mapped to hg38 using Bowtie 2.2.6.2 (Langmead and Steven L Salzberg, 2013) with default parameters. RNA-seq reads were mapped to hg38 transcriptome using Tophat2 (Kim et al., 2013), with default parameters, except for the following: library type=fr-firststrand, mate-std-dev=100, mate--inner-dist=300. For ChIP-seq and ATAC-seq duplicates were removed using samtools rmdup command (Li et al., 2009). ChIP-seq peaks were called using MACS2.1 (Zhang et al., 2008), with default parameters. For ATAC-seq, Fseq1.85 (Boyle et al., 2008) with default parameters was used, except for $f=50$. RNA-seq counts on genes and transcripts were calculated using samtools. Mapping and peak-calling with MACS were performed using Galaxy wrappers.

Defining active promoters and putative enhancers. Promoters were defined as region $+/-1000 \mathrm{bp}$ around every TSS. We presumed that a promoter has a H3K4me 3 mark in a patient $\mathrm{A}$ if it overlaps with any $\mathrm{H} 3 \mathrm{~K} 4 \mathrm{me} 3$ peak called in a patient $A$; the samea gene has a H3K4me3 mark if any of its promoters has a H3K4me3 mark. Similarly presumptions were made for H3K27ac marks and ATACseq signal. Overlaps were found using bedtools intersect tool (Quinlan and Hall, 2010). For every patient non-promoter H3K27ac peaks were defined as peaks that have no overlap with regions +/$1000 \mathrm{bp}$ from any TSS. Putative enhancers were defined by merging all non-promoter peaks identified for each patient (individual peak files were merged into a single file and overlapping peaks were merged into one peak using bedtools merge tool). Each putative enhancer was denoted as active in a patient $A$ if it overlapped with any non-promoter H3K27ac peak present in a patient $A$.

Common and variable marks. Active genes, promoters and enhancers were divided into two groups: common, which are present in at least threshold patients, and variable, which are present in less than threshold patients. Threshold value was chosen separately for every mark/type of genomic element. For promoters and genes with $\mathrm{H} 3 \mathrm{~K} 4 \mathrm{me} 3$ mark, threshold $=20$. For promoters and genes with H3K27ac mark, threshold $=10$. For enhancers with H3K27ac mark, threshold $=4$.

Coverage. Coverages over whole genome were calculated using bedtools genomecov tool. Coverages over specific intervals were calculated using bedtools intersect tool and custom python script calculate_coverage_over_features.py. Briefly, for every interval sum of coverages over all 
positions were calculated; then the sum was divided by length of the interval to obtain mean coverage value. Normalized coverages were obtained by dividing every coverage value by mean coverage defined as summaric coverage (the sum of values on every position of the genome, including ones with no coverage) divided by the length of the genome.

Evolutionary conservation analysis. A collection of random genomic intervals was generated for both common active promoters and common active enhancers using bedtools shuffle. PhastCons 100-Way scores were obtained from http://hgdownload.cse.ucsc.edu/goldenPath/hg38/phastCons100way/hg38.phastCons100way.bw Mean PhastCons scores for all analyzed intervals were calculated with bigWigAverageOverBed from the kentUtils package (https://github.com/ENCODE-DCC/kentUtils). One-sided Mann-Whitney U test was calculated to assess significance of difference in conservation scores between identified regulatory elements and random genomic intervals.

Identification of enhancer-promoter contacts based on $\mathrm{Hi}-\mathrm{C}$ data. $\mathrm{Hi}-\mathrm{C}$ data for fetal brain samples from (Won et al., 2016) were used to infer long-range chromatin interactions for putative enhancers defined based on the presence of non-promoter H3K27ac peaks. First, coordinates of putative enhancers were converted from hg38 to hg19 using UCSC liftover tool, to match the Hi-C data. Then the HiCEnterprise method (https://github.com/hansiu/HiCEnterprise) was used to identify contacts within $2 \mathrm{Mb}$ distance with $10 \mathrm{~kb}$ resolution (Kranas et al., 2019). Coordinates of identified contacts were converted back to hg38.

Correlation between histone marks on promoters and transcript expression. H3K4me 3 and H3K27ac ChIP-seq coverages on promoter regions and RNA-seq counts on transcripts were normalized with quantile normalization. Only transcripts of protein-coding genes were considered. For genes with multiple alternative transcripts, the transcript with a highest ChIP-seq coverage on promoter was chosen. Spearman correlation between ChIP-seq coverage and transcript expression was calculated for all transcript-promoter pairs in all patients. For violin plot visualization, transcripts were divided into quantiles based on expression level (quantile normalized counts).

Correlation between H3K27ac level on enhancers and transcript expression. For each enhancer identified by the presence of H3K27ac mark, the closest transcript was found using bedtools closest. The enhancers were subsequently divided into three groups: i) having the closest transcript within less than 20 kilobases, ii) having closest transcript within 20 to $200 \mathrm{~kb}$, iii) having closest transcript located more than $200 \mathrm{~kb}$ away. Spearman correlation between H3K27ac coverage and transcript expression was calculated for each of these groups of enhancers. Then, enhancers assigned to each group were checked for contacts with promoters, predicted from Hi-C data, and Spearman correlation was again calculated for enhancer-transcript pairs defined with $\mathrm{Hi}-\mathrm{C}$. In cases where one enhancer had predicted contacts with more than one promoter, mean of counts for transcripts associated with all contacting promoters was used in the correlation calculation. P-value for the difference between correlation obtained for closest enhancer-transcript pairs and pairs defined with $\mathrm{Hi}-\mathrm{C}$ data was estimated by random sampling 260 enhancer-transcript pairs from the set of 3371 enhancer-transcript pairs with closest transcript located further than $200 \mathrm{~kb}$ away for 1000 times.

From all enhancer-promoter pairs predicted based on $\mathrm{Hi}-\mathrm{C}$ data pairs with significant correlation between H3K27ac coverage and transcript expression were chosen based on the following criteria: 
FDR $<0.1$, Spearman $R>0.7$, minimal read counts on transcript in a sample with highest expression $=20$. Amongst analyzed pairs, 109 enhancer-promoter pairs fulfilled these criteria.

Clustering based on gene expression. RNA-seq row counts on genes of 37 cases (4 NB, 11 PA, 7DA, 14GB, $1 \mathrm{PG}$ ) were plotted for each tumor grade and hospital of a sample origin. No batch effect related to grade or hospital was detected(Risso et al., 2011). Two normalization procedures were considered following recommendations (Risso et al., 2011): (i) within-lane to adjust for GC-content and gene-length; (ii) between-lane, both implemented in EDASeq 3.8 R package (Risso et al., 2015). Genes with mean row counts across all samples below 10 were filtered out, and then the EDASeq 3.8 protocol was applied with full-quantile normalization method for within- and between-lane normalization. The filtering step resulted in 19,328 genes that were used for clustering. Dendextend $R$ package (Galili, 2015) was used to perform hierarchical clustering with ward.D2 method with distance set to Euclidean. ssGSEA tool (gene-centric single sample Gene Set Enrichment Analysis of gene expression data) was used to define GBM subtypes (downloaded from https://github.com/broadinstitute/ssGSEA2.0) Input data for the analysis consisted 37 samples and 56,819 RPKM normalized gene expression values, converted to GCT file format. Additionally, four reference files (for each subtype separately) have been downloaded from Molecular Signatures Database v7.0 (http://software.broadinstitute.org/gsea/msigdb/index.jsp) and used in the analysis. To predict the subtype of Pan Glioma RNA Expression Cluster (LGr1, LGr2, LGr3, LGr4), a validation cohort from published studies, including 665 adult and pediatric gliomas (Ceccarelli et al., 2016) and 21,022 of their gene expressions levels were compiled. Next on that data we trained three classifiers (Naïve Bayes, SVM and Random Forest) and applied them as a committee of classifiers with voting mechanism on our 26 samples to predict the target variable (Pan Glioma Cluster).

Gene expression differential analyses were computed with edgeR Bioconductor package. For visualization and co-activation analyses FPKM (Fragments per Kilobase Million) values were calculated.

Comparison of ChIP-seq and ATAC-seq profiles in TSS-proximal regions. Genomic positions with the numbers of mapped reads above the significance threshold of $z$-score $=7$ were identified as anomalous, and the tags mapped to such positions were discarded. Read frequencies were computed in 500-bp non-overlapping bins for each sample independently and normalized by the corresponding library sizes to represent values per one million of mapped reads. For the histone marks abundance was computed per bin as a difference between normalized frequencies obtained from chip and input experiments. For each TSS proximal region, the abundance values were computed as an average over abundance in bins overlapping the corresponding $4 \mathrm{~kb}$ region (TSS+/$2 \mathrm{~kb}$ ). The transcription start site proximal regions were defined as $+/-2 \mathrm{~kb}$ from the TSS. TSS proximal regions overlapping with other genes were excluded from this analysis. The averaged and detailed profiles around TSS were computed using 10 bp non-overlapping bins and smoothed using runmean function from caTools package with $\mathrm{k}=15$.

Clustering based on H3K4me3 mark. For each sample a set of promoters with $\mathrm{H} 3 \mathrm{~K} 4 \mathrm{me} 3$ mark was defined based on a presence of H3K4me3 ChIP-seq peak as described above. Pairwise similarities between samples were defined as Jaccard indices calculated for sets of promoters with H3K4me3 mark. These pairwise similarities were subsequently used to perform hierarchical clustering of 
samples with scipy.cluster.hierarchy.linkage function and the UPGMA algorithm (method = "average").

Identification of promoters specifically active/inactive in the PAs cluster. To identify promoters whose activity differentiate PA from higher grade glioma, for each annotated promoter we compared the number of samples from the PA cluster in which it was active (marked with H3K4me3) to the number of samples from a group of 13 higher grade samples (DA01, DA03, DA04, DA05, DA06, GB03, GB04, GB05, GB06, GB07, GB08, GB09, GB10) in which it was active. As specifically active in PA, we chose those active in at least four out of five samples from the PA cluster and in at most two higher grade samples and additionally having p-value from Fisher's exact test below 0.003 . Similarly, promoters specifically active in higher grades were defined as those active in at most one PA sample and at least 11 higher grades samples, and having the p-value below 0.003 .

Enrichment analysis for DNA-binding proteins binding sites. The ENCODE Regulation 'Txn Factor' track data was downloaded from http://hgdownload.soe.ucsc.edu/goldenPath/hg19/encodeDCC/wgEncodeRegTfbsClustered/ promoters specifically active in PAs, active in all samples or inactive in any sample were intersected with bed files defining binding sites of 161 proteins using bedtools intersect. Enrichments in binding sites were calculated as a fold change of the number of intersections between each protein binding sites and promoters specifically active in PAs and number of intersections for the same protein binding sites and active or inactive promoters (per megabase).

Protein binding motif analysis. To identify transcription factors that can potentially bind to the identified promoters with $\mathrm{H} 3 \mathrm{~K} 27$ ac hyperacetylation or H3K4me3 hypermethylation in PA, we used HOCOMOCO-v11 motif database (Kulakovskiy et al., 2018). For hyperacetylated promoters the relative motif enrichment was computed with AME tool from MEME-Suite software (v5.0.4) [10.1186/1471-2105-11-165] and logos were obtained from the HOCOMOCO website. For hypermethylated promoters, motif enrichment was computed with PWMEnrich R package (Stojnic and Diez, 2013) using sequences of promoters inactive in all samples as background. The returned protein binding motifs were accepted if their $p$-value was below 0.001 . To establish the similarity of motifs enriched for grade-specific groups (PAs vs. higher grade gliomas - HGG) on each of the backgrounds, the Jaccard index (Jaccard, 1901) implemented in 'jaccard' CRAN R package (Christopher Chung et al.) was used.

Analysis of enriched Gene Ontology terms. Enrichment of gene ontology terms for promoters with H3K4me3 hypermethylation in PAs and EZH2 binding sites was performed using the PANTHER Overrepresentation Test (Released 20171205) (Ashburner et al., 2000; Carbon et al., 2017) and Fisher's Exact with FDR multiple test correction. For promoters with H3K27ac hyperacetylation in PAs, gene ontology analysis was performed with GO.db Bioconductor package and 0.05 significance threshold on Bonferroni corrected P-value (Fisher's exact test) was used.

DNA methylation data processing. For DNA methylation, assessment of methylation sites was restricted to regions covered by SeqCap Epi CpGiant Methylation panel which captures $80.5 \mathrm{Mb}$ (Roche). The methylation analysis workflow consisted of: read quality assessment, mapping to hg38 genome, removal of PCR duplicates, coverage statistics assessment and methylation level assignment. The software required to perform the aforementioned steps: FastQC, BSMAP (Xi and Li, 2009), Picard ( http://broadinstitute.github.io/picard/) were selected based on Roche recommended 
pipeline (NimbleGen, 2014) and compiled into a single CytoMeth tool (https://github.com/mdraminski/ CytoMeth) enabling fast and transparent rough data processing. The following quality requirements were set: minimal bisulphite conversion $99.2 \%$ and at least 3, 000, 000 cytosines in a CpG context covered with at least ten reads. Out of 26 samples, 20 passed these requirements and were used to assess DNA methylation variability within promoter regions (+/-2000 bp from TSS) with a use of methylKit (Akalin et al., 2012).

For Infinium HumanMethylation 450 BeadChip data signal intensities were imported into R using the methylumi package [10.1093/bib/bbt054]. Initial quality control checks were performed using functions in the methylumi package. The methylation score for each CpG was represented as a $\beta$-value according to the fluorescent intensity ratio representing any value between 0 (unmethylated) and 1 (completely methylated). Mapping of CpGs was performed based on UCSC Genome Browser annotation data (Haeussler et al., 2019). CpGs beta values in the range from 0 to 0.2 were defined as hypomethylated and those from 0.8 to 1 as hypermethylated.

DNA methylation analysis for PAs specific promoters with EZH2 binding sites. Signal intensities for probes from HumanMethylation450 BeadChip array, which were located within PA specific promoters with EZH2 binding sites, were compared for 11 independent PAs and 13 GB samples. DNA methylation data from previously pubslihed studies (Lambert et al., 2013), and gene expression data from the altter were downloaded from Gene Expression Omnibus (GEO: http://www.ncbi.nlm.nih.gov/geo/) using the following accession numbers: GSE61431, GSE44684 and GSE44971, respectively. Quantile normalization was performed on signal intensities. For the expression data median intensities were calculated for each probe in each analyzed group of samples.

TCGA data analyses. The expression values for the TCGA samples were downloaded from TCGA website (RNASeqV2 set). Survival analyses were performed with TCGA data. The patients were stratified into three equinumerous sub-groups according to $A N X A 2 R$ or FOXM1 expression levels. Correlation between the expression level of the selected genes and patient survival times was analyzed using Kaplan-Meier model and log-rank test. 


\section{REFERENCES}

Afgan, E., Baker, D., van den Beek, M., Blankenberg, D., Bouvier, D., Čech, M., Chilton, J., Clements, D., Coraor, N., Eberhard, C., et al. (2016). The Galaxy platform for accessible, reproducible and collaborative biomedical analyses: 2016 update. Nucleic Acids Res. 44, W3-W10.

Akalin, A., Kormaksson, M., Li, S., Garrett-Bakelman, F.E., Figueroa, M.E., Melnick, A., and Mason, C.E. (2012). MethylKit: a comprehensive R package for the analysis of genome-wide DNA methylation profiles. Genome Biol. 13.

Ashburner, M., Ball, C.A., Blake, J.A., Botstein, D., Butler, H., Cherry, J.M., Davis, A.P., Dolinski, K., Dwight, S.S., Eppig, J.T., et al. (2000). Gene ontology: Tool for the unification of biology. Nat. Genet. 25, 25-29.

Bibikova, M., Barnes, B., Tsan, C., Ho, V., Klotzle, B., Le, J.M., Delano, D., Zhang, L., Schroth, G.P., Gunderson, K.L., et al. (2011). High density DNA methylation array with single CpG site resolution. Genomics 98, 288-295.

Blankenberg, D., Gordon, A., Von Kuster, G., Coraor, N., Taylor, J., Nekrutenko, A., and Team, G. (2010). Manipulation of FASTQ data with galaxy. Bioinformatics 26, 1783-1785.

Bolger, A.M., Lohse, M., and Usadel, B. (2014). Trimmomatic: A flexible trimmer for Illumina sequence data. Bioinformatics 30, 2114-2120.

Boyle, A.P., Guinney, J., Crawford, G.E., and Furey, T.S. (2008). F-Seq: A feature density estimator for high-throughput sequence tags. Bioinformatics 24, 2537-2538.

Buenrostro, J.D., Giresi, P.G., Zaba, L.C., Chang, H.Y., and Greenleaf, W.J. (2013). Transposition of native chromatin for fast and sensitive epigenomic profiling of open chromatin, DNA-binding proteins and nucleosome position. Nat. Methods 10, 1213-1218.

Carbon, S., Dietze, H., Lewis, S.E., Mungall, C.J., Munoz-Torres, M.C., Basu, S., Chisholm, R.L., Dodson, R.J., Fey, P., Thomas, P.D., et al. (2017). Expansion of the Gene Ontology knowledgebase and resources. Nucleic Acids Res. 45, D331-D338.

Ceccarelli, M., Barthel, F.P., Malta, T.M., Sabedot, T.S., Salama, S.R., Murray, B.A., Morozova, O., Newton, Y., Radenbaugh, A., Pagnotta, S.M., et al. (2016). Molecular Profiling Reveals Biologically Discrete Subsets and Pathways of Progression in Diffuse Glioma. Cell 164, 550-563.

Christopher Chung, N., Miasojedow, B., and Gambin, A. Pattern Recognition of Chemical Fingerprints from Large-Scale Databases of Molecules and Reactions.

Galili, T. (2015). dendextend: an R package for visualizing, adjusting and comparing trees of hierarchical clustering. Bioinformatics 31, 3718-3720.

Haeussler, M., Zweig, A.S., Tyner, C., Speir, M.L., Rosenbloom, K.R., Raney, B.J., Lee, C.M., Lee, B.T., Hinrichs, A.S., Gonzalez, J.N., et al. (2019). The UCSC Genome Browser database: 2019 update. Nucleic Acids Res. 47, D853-D858.

Jaccard, P. (1901). Étude comparative de la distribution florale dans une portion des Alpes et du Jura. Bull. La Société Vaudoise Des Sci. Nat. 37, 547-579.

Kim, D., Pertea, G., Trapnell, C., Pimentel, H., Kelley, R., and Salzberg, S.L. (2013). TopHat2: Accurate alignment of transcriptomes in the presence of insertions, deletions and gene fusions. Genome Biol. 14. 
Kranas H, Tuszyńska I, Wilczynski B. 2019. HiCEnterprise: Identifying long range chromosomal contacts in HiC data. PeerJ Preprints 7:e27753v1 https://doi.org/10.7287/peerj.preprints.27753v1

Kulakovskiy, I. V., Vorontsov, I.E., Yevshin, I.S., Sharipov, R.N., Fedorova, A.D., Rumynskiy, E.I., Medvedeva, Y.A., Magana-Mora, A., Bajic, V.B., Papatsenko, D.A., et al. (2018). HOCOMOCO: towards a complete collection of transcription factor binding models for human and mouse via largescale ChIP-Seq analysis. Nucleic Acids Res. 46, D252-D259.

Lambert, S.R., Witt, H., Hovestadt, V., Zucknick, M., Kool, M., Pearson, D.M., Korshunov, A., Ryzhova, M., Ichimura, K., Jabado, N., et al. (2013). Differential expression and methylation of brain developmental genes define location-specific subsets of pilocytic astrocytoma. Acta Neuropathol. 126, 291-301.

Langmead and Steven L Salzberg (2013). Bowtie2. Nat. Methods 9, 357-359.

Li, H., Handsaker, B., Wysoker, A., Fennell, T., Ruan, J., Homer, N., Marth, G., Abecasis, G., Durbin, R., and 1000 Genome Project Data Processing Subgroup (2009). The Sequence Alignment/Map format and SAMtools. Bioinformatics 25, 2078-2079.

Mi, H., Huang, X., Muruganujan, A., Tang, H., Mills, C., Kang, D., and Thomas, P.D. (2017).

PANTHER version 11: expanded annotation data from Gene Ontology and Reactome pathways, and data analysis tool enhancements. Nucleic Acids Res. 45, D183-D189.

NimbleGen, R. (2014). How To... Evaluate NimbleGen SeqCap Epi Target Enrichment Data. 1-22.

Pidsley, R., Viana, J., Hannon, E., Spiers, H., Troakes, C., Al-Saraj, S., Mechawar, N., Turecki, G., Schalkwyk, L.C., Bray, N.J., et al. (2014). Methylomic profiling of human brain tissue supports a neurodevelopmental origin for schizophrenia. Genome Biol. 15, 483.

Quinlan, A.R., and Hall, I.M. (2010). BEDTools: a flexible suite of utilities for comparing genomic features. Bioinformatics 26, 841-842.

Risso, D., Schwartz, K., Sherlock, G., and Dudoit, S. (2011). GC-Content Normalization for RNA-Seq Data. BMC Bioinformatics 12.

Risso, D., Eda, R., and Eda, G. (2015). EDASeq: Exploratory Data Analysis and Normalization for. 116.

Stojnic, R., and Diez, D. (2013). PWMEnrich: PWM enrichment analysis. R Packag. Version.

Won, H., de la Torre-Ubieta, L., Stein, J.L., Parikshak, N.N., Huang, J., Opland, C.K., Gandal, M., Sutton, G.J., Hormozdiari, F., Lu, D., et al. (2016). Chromosome conformation elucidates regulatory relationships in developing human brain. Nature 538, 523-527.

Xi, Y., and Li, W. (2009). BSMAP: Whole genome bisulfite sequence MAPping program. BMC Bioinformatics 10.

Zhang, Y., Liu, T., Meyer, C.A., Eeckhoute, J., Johnson, D.S., Bernstein, B.E., Nussbaum, C., Myers, R.M., Brown, M., Li, W., et al. (2008). Model-based analysis of ChIP-Seq (MACS). Genome Biol. 9. 


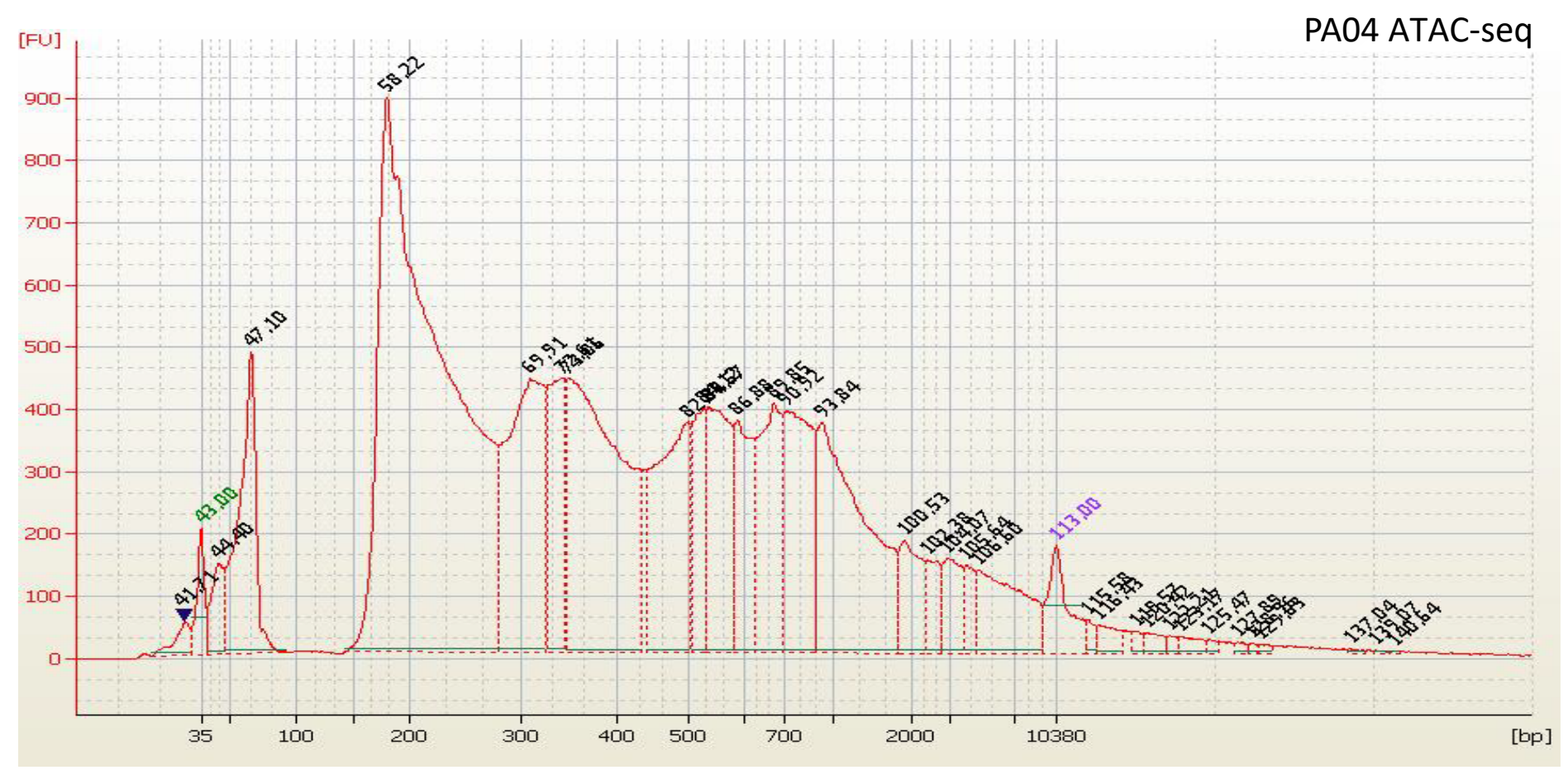

B
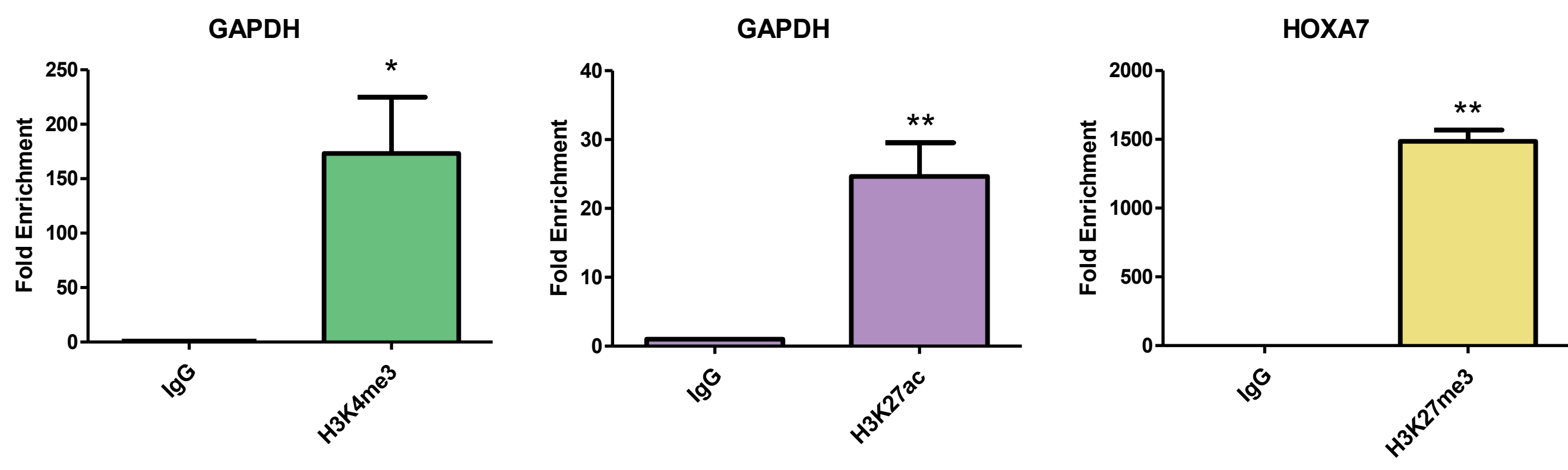


\section{Supplementary Figure 1}

A. A characteristic appearance of a single nucleosome and their multiplications in the material isolated for ATAC-seq separated on DNA Agilent chips (PA04 sample).

B. Validation of immunoprecipitation efficiency with antibodies used in the experiments (for details see Methods). Chromatin immunoprecipitation (ChIP)quantitative polymerase chain reaction (qPCR) analysis of histone modification enrichment in control regions: GAPDH promoter (active chromatin marks, H3K4me3, H3K27ac) and HOXA7 gene body (repressive mark, H3K27me3). Results are calculated as fold enrichment over negative control (immunoprecipitation with normal IgG) and represented as mean \pm SD (H3K4me3 ChIP-qPCR n=6, H3K27ac ChIPqPCR $n=5, H 3 K 27 m e 3$ ChIP-qPCR $n=3 n=4)$. Statistical significance ** $P<0.05 i{ }^{* *} P$ $<0.01$ (t-test). 
Enhancer ( $>=4$ patient) bins with predictions

Percentage of occurence in all enhancer bins for FDR threshold 0.01 BRAIN Hi-C maps (Won 2016)

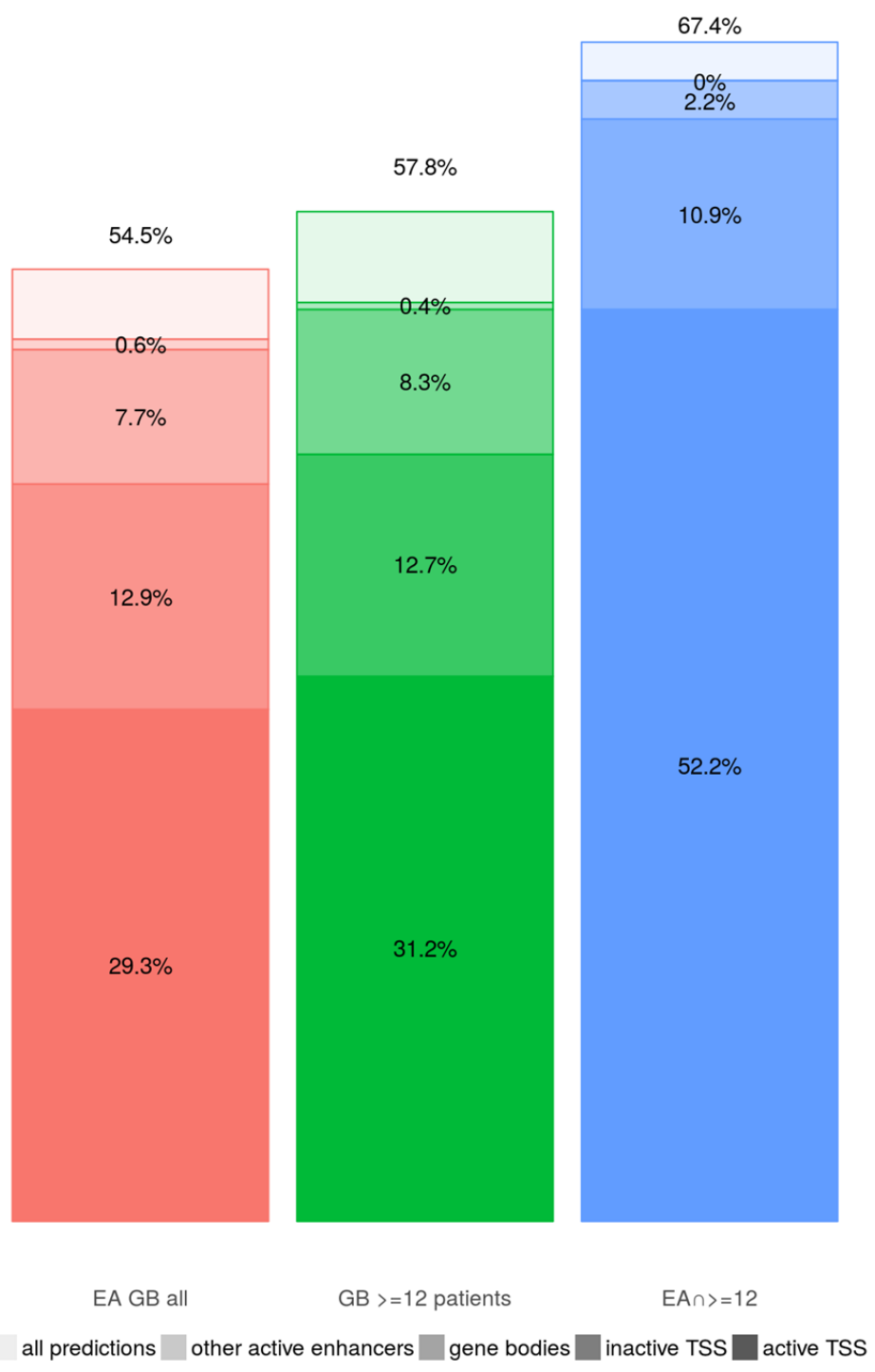

\section{Supplementary Figure 2}

Percentage of enhancer bins predicted to be in contact with different genomic elements. EA GB all - all enhancers reported in EnhancerAtlas (Gao et al. 2016) and in this work in at least 4 samples; GB $>=12$ patients - enhancers identified in this work in at least 12 samples; EA and $>=12$ - enhancers present both in EnhancerAtlas and at least 12 samples in this work. 


\section{Supplementary Figure 3}

A. Overlap of active genes and enhancers sets between patients. Colors indicate experimental method used to detect the activity.

B. Size of intersections between H3K27ac ChIP-seq peaks in active promoters and enhancers identified in this work (glioma-derived) and H3K27ac peaks from normal brain (control samples from Sun et al. 2016) or non-brain cell lines from the ENCODE project (Gerstein et al. 2012) vs total size of promoter/enhancer peaks detected in this work.

C. Comparison of RNA-seq results between different glioma Grades and hospitals where brain samples have been collected. 

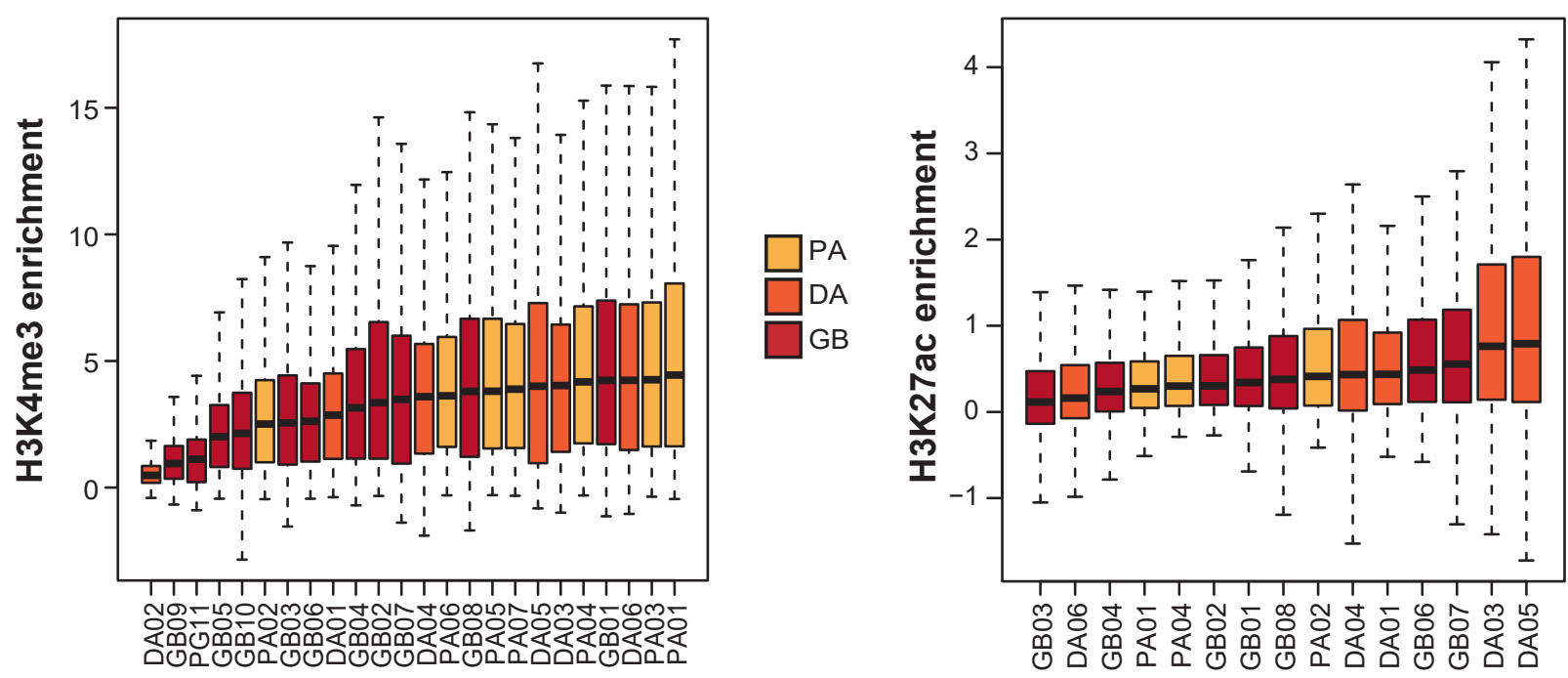

\section{Supplementary Figure 4}

Boxplots representing global enrichment in H3K4me3 (left) and H3K27ac (right) in the regions around TSS in every single glioma sample form our cohort order from lowest to highest median value. The colour coding has been used to discriminate between major malignancy groups. 
bioRxiv preprint doi: https://doi.org/101101/867861; this version posted March 20,2020. The copyright holder for this preprint (which was not certified by peer review) is the author/funder, who has granted bioRxiv a license to display the preprint in perpetuity. It is made

A

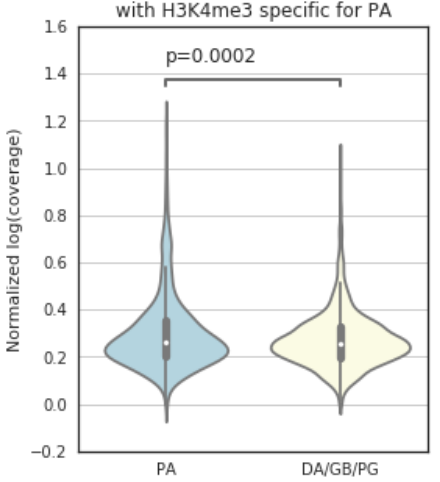

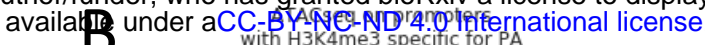

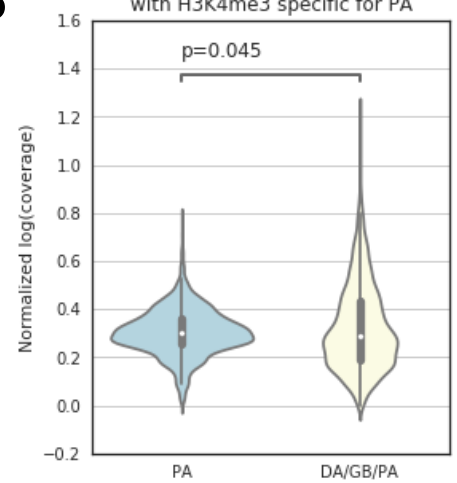

C
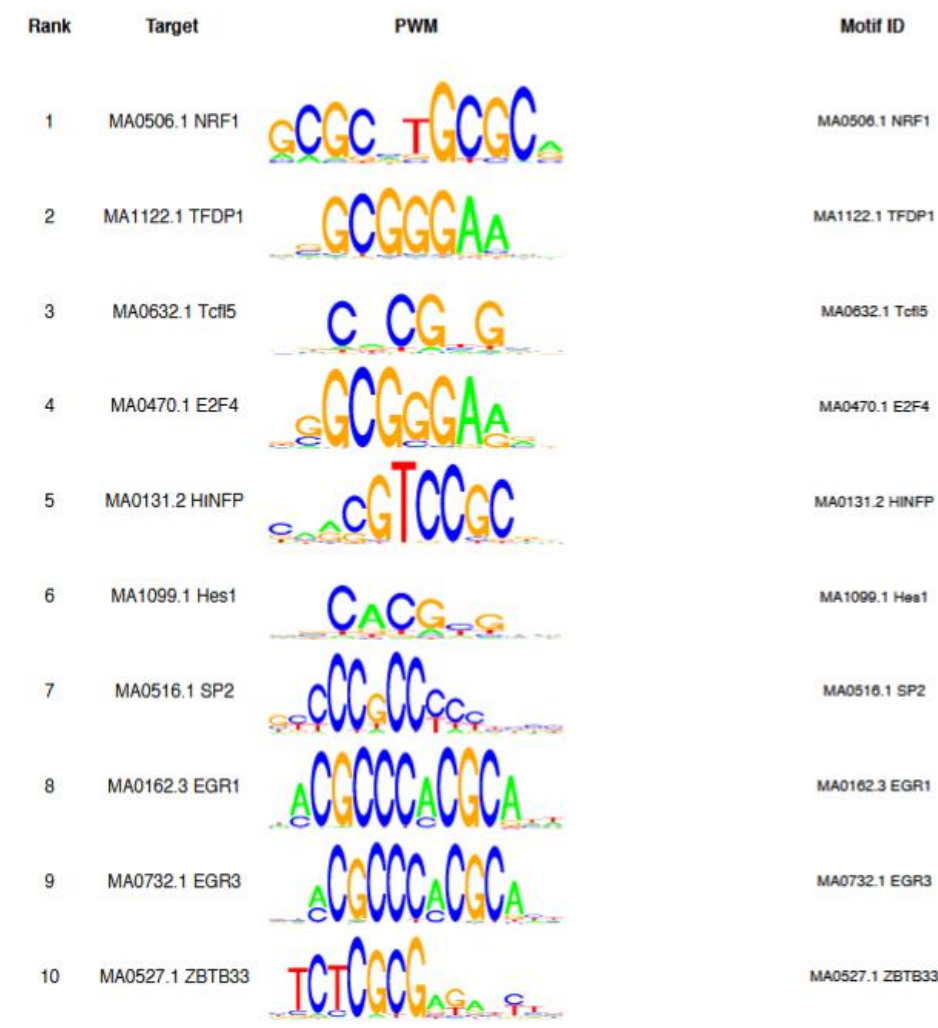

Raw score P-value In top

motifs

$17 \quad 6.82 \mathrm{e}-141 \quad 40 \%$

$3.89 \quad 6.32 \mathrm{e}-134 \quad 39 \%$

$1.44 \quad 2.24 \mathrm{e}-129 \quad 40 \%$

$6.23 \quad 4.86 \mathrm{e}-109 \quad 39 \%$

$2.51 \quad 2.46 \mathrm{e}-104 \quad 33 \%$

$1.37 \quad 4.43 e-102 \quad 32 \%$

$95 \quad 8.94 \mathrm{e}-101 \quad 31 \%$

$1.74 \quad 2 e-98 \quad 28 \%$

$4.99 \quad 4.81 e-93 \quad 27 \%$

$1.14 \quad 1.88 \mathrm{e}-80 \quad 26 \%$

D

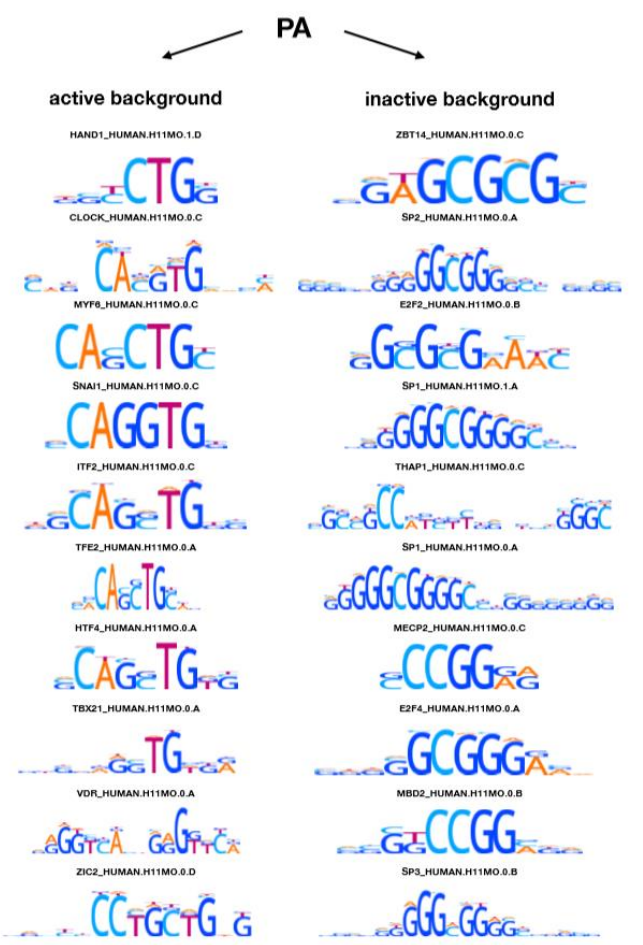

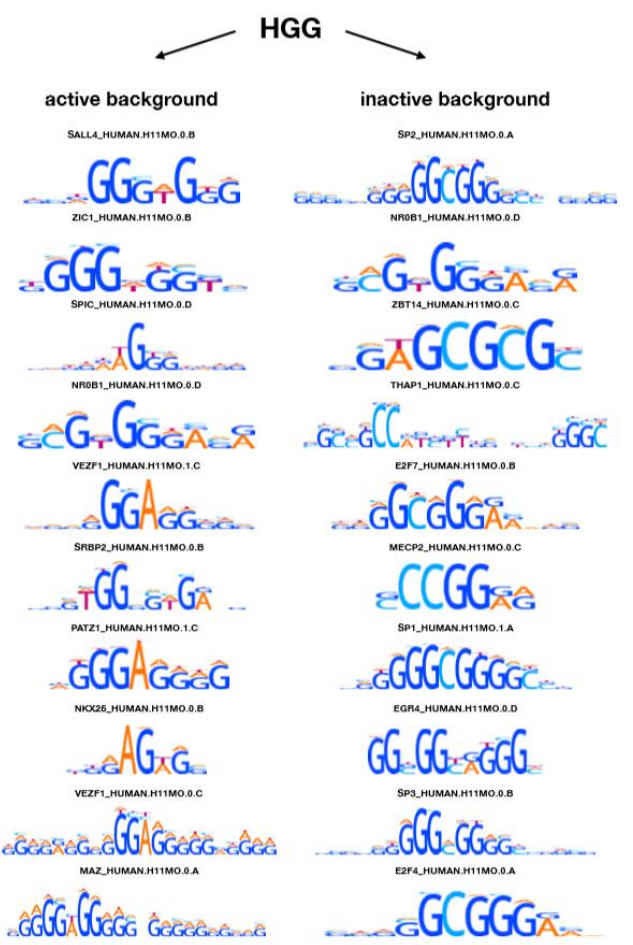


bioRxiv preprint doi: https://doi.org/10.1101/867861; this version posted March 20, 2020. The copyright holder for this preprint (which was not certified by peer review) is tha author (fupheder, who has granted (biokxiva license to display the preprint in perpetuity. It is made

E GO biological process complete available under aCC-BY-NC-ND 4 . International licesse.

\begin{tabular}{|c|c|c|c|c|c|c|c|}
\hline locomotory behavior & 192 & $\underline{8}$ & .85 & 9.43 & + & $2.86 \mathrm{E}-06$ & 4.06E-03 \\
\hline 4 behavior & $\underline{555}$ & 12 & 2.45 & 4.89 & + & 7.14E-06 & 7.43E-03 \\
\hline sodium ion transport & 169 & 7 & .75 & 9.37 & + & $1.28 \mathrm{E}-05$ & 1.17E-02 \\
\hline 4 monovalent inorganic cation transport & $\underline{415}$ & 10 & 1.83 & 5.45 & + & $1.78 \mathrm{E}-05$ & 1.54E-02 \\
\hline 4 cation transport & $\underline{819}$ & 16 & 3.62 & 4.42 & + & $6.74 \mathrm{E}-07$ & 1.76E-03 \\
\hline 4ion transport & 1284 & 18 & 5.67 & 3.17 & + & $1.28 \mathrm{E}-05$ & 1.25E-02 \\
\hline 4 metal ion transport & $\underline{630}$ & 16 & 2.78 & 5.75 & + & 2.07E-08 & $1.08 \mathrm{E}-04$ \\
\hline sensory_perception of sound & 148 & $\underline{6}$ & .65 & 9.17 & + & $6.14 \mathrm{E}-05$ & 4.36E-02 \\
\hline Lnervous system process & 1335 & 17 & 5.90 & 2.88 & + & $7.59 \mathrm{E}-05$ & 4.94E-02 \\
\hline ᄂsystem process & 1893 & $\underline{21}$ & 8.37 & 2.51 & + & $6.96 \mathrm{E}-05$ & 4.73E-02 \\
\hline 4 multicellular organismal process & $\underline{6695}$ & $\underline{58}$ & 29.59 & 1.96 & + & $1.90 \mathrm{E}-09$ & $1.48 \mathrm{E}-05$ \\
\hline inorganic cation transmembrane transport & $\underline{569}$ & 13 & 2.51 & 5.17 & + & $1.58 \mathrm{E}-06$ & $3.09 \mathrm{E}-03$ \\
\hline${ }^{4}$ cation transmembrane transport & $\underline{613}$ & 13 & 2.71 & 4.80 & + & $3.52 \mathrm{E}-06$ & 4.23E-03 \\
\hline Lion transmembrane transport & $\underline{886}$ & 16 & 3.92 & 4.09 & + & 1.85E-06 & $3.21 \mathrm{E}-03$ \\
\hline Ltransmembrane transport & 1208 & 17 & 5.34 & 3.18 & + & $2.20 \mathrm{E}-05$ & $1.72 \mathrm{E}-02$ \\
\hline Linorganic ion transmembrane transport & $\underline{650}$ & 14 & 2.87 & 4.87 & + & $1.20 \mathrm{E}-06$ & 2.67E-03 \\
\hline epithelium development & 1075 & 16 & 4.75 & 3.37 & + & 2.04E-05 & 1.67E-02 \\
\hline Lanatomical structure development & $\underline{5189}$ & $\underline{46}$ & 22.93 & 2.01 & + & $3.50 \mathrm{E}-07$ & $1.09 \mathrm{E}-03$ \\
\hline 4 developmental process & $\underline{5527}$ & $\underline{52}$ & 24.43 & 2.13 & + & $1.80 \mathrm{E}-09$ & $2.81 \mathrm{E}-05$ \\
\hline cell differentiation & $\underline{3574}$ & $\underline{35}$ & 15.80 & 2.22 & + & 2.27E-06 & $3.54 \mathrm{E}-03$ \\
\hline 4cellular developmental process & $\underline{3665}$ & $\underline{35}$ & 16.20 & 2.16 & + & $3.46 \mathrm{E}-06$ & 4.50E-03 \\
\hline animal organ development & $\underline{3077}$ & $\underline{29}$ & 13.60 & 2.13 & + & $4.78 \mathrm{E}-05$ & 3.55E-02 \\
\hline 4s system development & $\underline{4244}$ & $\underline{38}$ & 18.76 & 2.03 & + & $6.15 \mathrm{E}-06$ & $6.86 \mathrm{E}-03$ \\
\hline$\stackrel{4 m u l t i c e l l u l a r}{\text { organism development }}$ & $\underline{4820}$ & $\underline{46}$ & 21.30 & 2.16 & + & 3.27E-08 & $1.28 \mathrm{E}-04$ \\
\hline
\end{tabular}

\begin{tabular}{|c|c|c|c|c|c|c|c|}
\hline & Homo sapiens (REF) & & & pload_1 $1 \nabla$ Hierar & rchy_ & - NEW! (?) & \\
\hline GO molecular function complete & \# & \# & expected & Fold Enrichment & $+1-$ & raw $P$ value & FDR \\
\hline luteinizing hormone receptor activity. & $\underline{2}$ & $\underline{2}$ & .01 & $>100$ & + & $1.14 \mathrm{E}-04$ & $3.80 \mathrm{E}-02$ \\
\hline catecholamine binding & 19 & $\underline{3}$ & .08 & 35.72 & + & $1.20 \mathrm{E}-04$ & 3.71E-02 \\
\hline solute:sodium symporter activity. & $\underline{68}$ & $\underline{6}$ & .30 & 19.96 & + & 8.98E-07 & 1.39E-03 \\
\hline 4 sodium ion transmembrane transporter activity. & 147 & $\underline{7}$ & .65 & 10.77 & + & $5.33 \mathrm{E}-06$ & 4.14E-03 \\
\hline 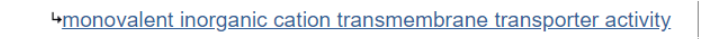 & $\underline{380}$ & 10 & 1.68 & 5.95 & + & 8.45E-06 & 4.37E-03 \\
\hline Linorganic cation transmembrane transporter activity. & $\underline{585}$ & 14 & 2.59 & 5.41 & + & $3.51 \mathrm{E}-07$ & 8.16E-04 \\
\hline 4 cation transmembrane transporter activity. & $\underline{641}$ & 14 & 2.83 & 4.94 & + & $1.02 \mathrm{E}-06$ & $1.18 \mathrm{E}-03$ \\
\hline Lion transmembrane transporter activity. & $\underline{896}$ & 15 & 3.96 & 3.79 & + & 9.89E-06 & $4.60 \mathrm{E}-03$ \\
\hline 4 transmembrane transporter activity. & 1066 & 16 & 4.71 & 3.40 & + & 1.84E-05 & 7.13E-03 \\
\hline Ltransporter activity. & 1261 & 18 & 5.57 & 3.23 & + & $1.00 \mathrm{E}-05$ & 4.24E-03 \\
\hline Linorganic molecular entity transmembrane transporter activity. & $\underline{839}$ & 15 & 3.71 & 4.05 & + & 4.56E-06 & 4.24E-03 \\
\hline 4 metal ion transmembrane transporter activity. & $\underline{432}$ & 14 & 1.91 & 7.33 & + & $9.21 \mathrm{E}-09$ & 4.29E-05 \\
\hline ¿solute:cation symporter activity. & $\underline{99}$ & $\underline{6}$ & .44 & 13.71 & + & $6.99 \mathrm{E}-06$ & 4.65E-03 \\
\hline 4 symporter activity. & 134 & $\underline{6}$ & .59 & 10.13 & + & $3.60 \mathrm{E}-05$ & 1.29E-02 \\
\hline Lsecondary active transmembrane transporter activity. & $\underline{219}$ & $\underline{8}$ & .97 & 8.27 & + & $7.28 \mathrm{E}-06$ & 4.24E-03 \\
\hline
\end{tabular}

\begin{tabular}{|c|c|c|c|c|c|c|c|}
\hline & Homo sapiens (REF) & & & upload_1 $(\nabla$ Hierar & rchy & NEW! (?) & \\
\hline $\begin{array}{l}\text { GO cellular component } \\
\text { complete }\end{array}$ & \# & $\#$ & expected & Fold Enrichment & $+1-$ & raw $P$ value & $\underline{F D R}$ \\
\hline synapse part & $\underline{745}$ & 12 & 3.29 & 3.64 & + & 1.19E-04 & 4.74E-02 \\
\hline plasma membrane region & 1095 & 15 & 4.84 & 3.10 & + & $9.62 \mathrm{E}-05$ & 4.78E-02 \\
\hline ५plasma membrane part & $\underline{2836}$ & $\underline{28}$ & 12.53 & 2.23 & + & $2.69 \mathrm{E}-05$ & 5.36E-02 \\
\hline ५plasma membrane & $\underline{5592}$ & $\underline{43}$ & 24.72 & 1.74 & + & $5.03 \mathrm{E}-05$ & 3.34E-02 \\
\hline 4 cell periphery. & $\underline{5712}$ & 44 & 25.25 & 1.74 & + & $3.44 \mathrm{E}-05$ & $3.42 \mathrm{E}-02$ \\
\hline
\end{tabular}

$\mathrm{F}$
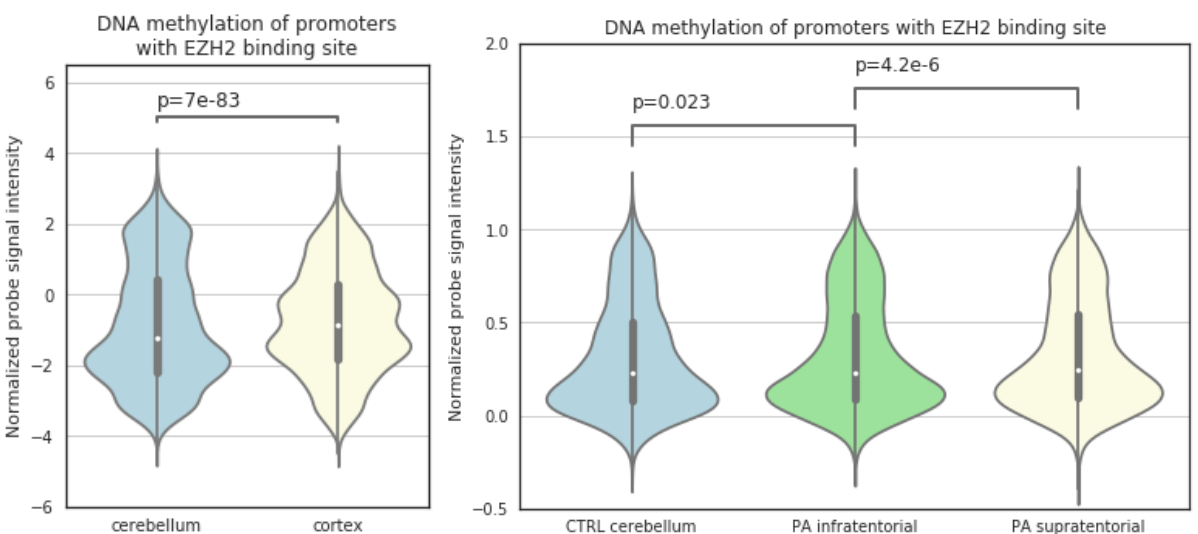

Expression of transcripts for promoters with EZH2 binding site

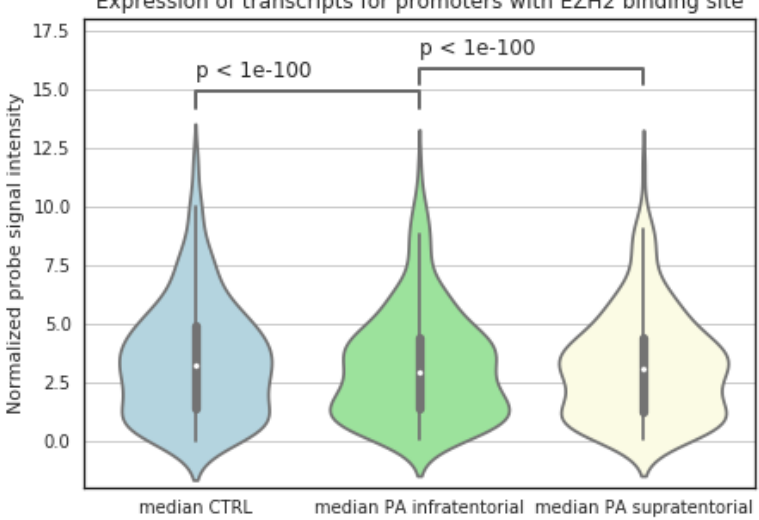




\section{Supplementary Figure 5}

A. H3K27ac ChIP-seq signal on promoters for which H3K4me3 presence is specific for PA compared for PA and higher grade samples.

B. ATAC-seq signal on promoters for which $\mathrm{H} 3 \mathrm{~K} 4 \mathrm{me} 3$ presence is specific for PA compared for PA and higher grade samples.

C. Transcription factor motifs enrichment in the promoters specifically active in PA compared to promoters inactive in all samples.

D. Transcription factor motifs enrichment in the promoters specifically active in PA or higher Grade gliomas (HGG) compared to promoters inactive in all samples (inactive background) or active in all samples (active background).

E. Enrichment of GO terms calculated for genes specifically active in PA and having the $\mathrm{EZH} 2$ protein binding site in promoters.

F. DNA methylation levels on promoters specifically active in PA and having the EZH2 protein binding site. Comparison between control cerebellum and cortex (based on Pidsley et al. 2014)

G. DNA methylation levels on promoters specifically active in PA and having the EZH2 protein binding site. Comparison between control cerebellum and pilocytic astrocytomas located in infra- or supratentorial brain regions (based on Lambert et al. 2013)

$H$. Expression of transcripts related with promoters specifically active in PA and having the $\mathrm{EZH} 2$ protein binding site. Comparison between control cerebellum and pilocytic astrocytomas located in infra- or supratentorial brain regions (based on Lambert et al. 2013).

All p-values were calculated with the one-sided Mann-Whitney $U$ test. 
bioRxiv preprint doi: https://doi.org/10.1101/867861; this version posted March 20, 2020. The copyright holder for this preprint (which was not certified by peer review) is the author/funder, who has granted bioRxiv a license to display the preprint in perpetuity. It is made

A
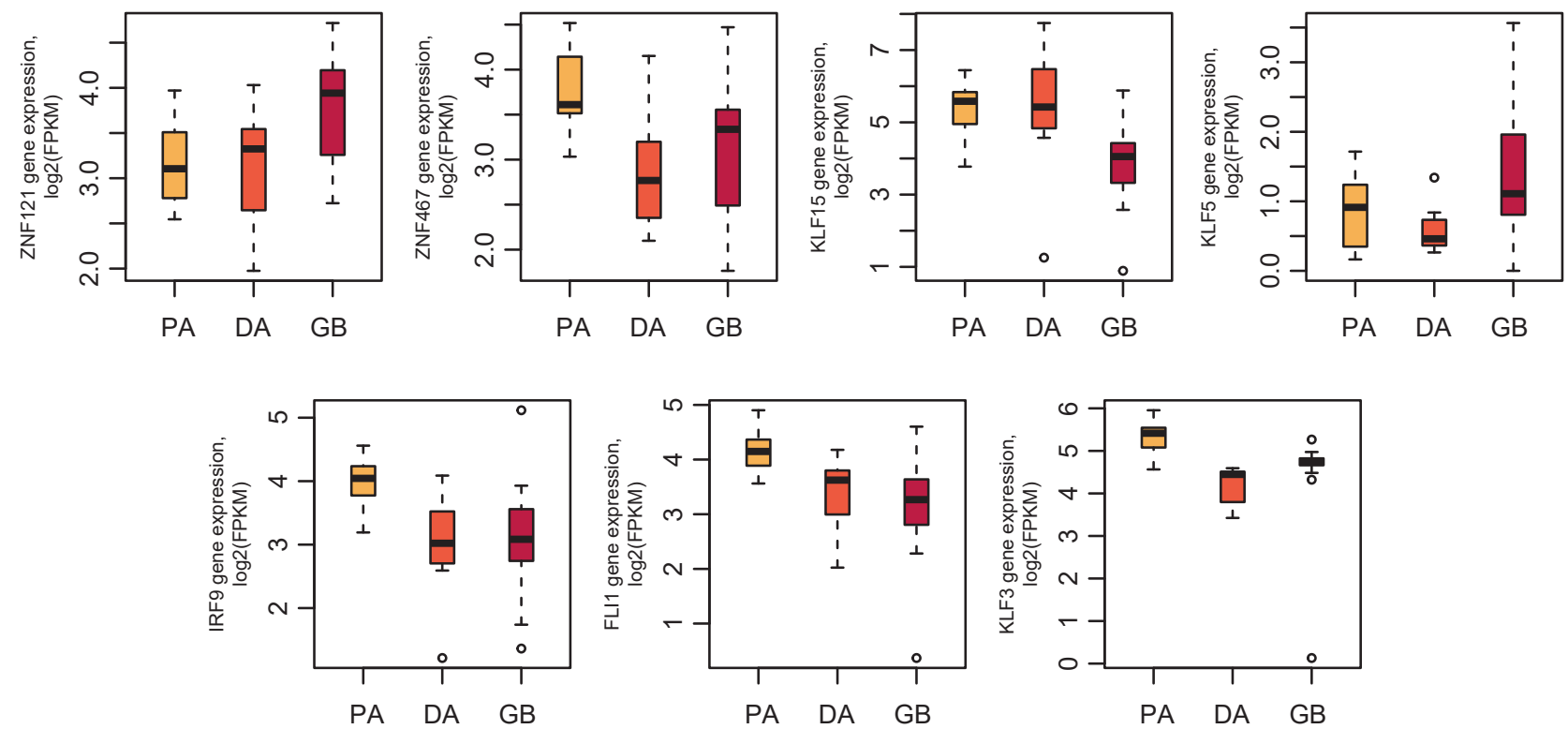

Patients stratified by

ANXA2R expression levels, only LGG samples

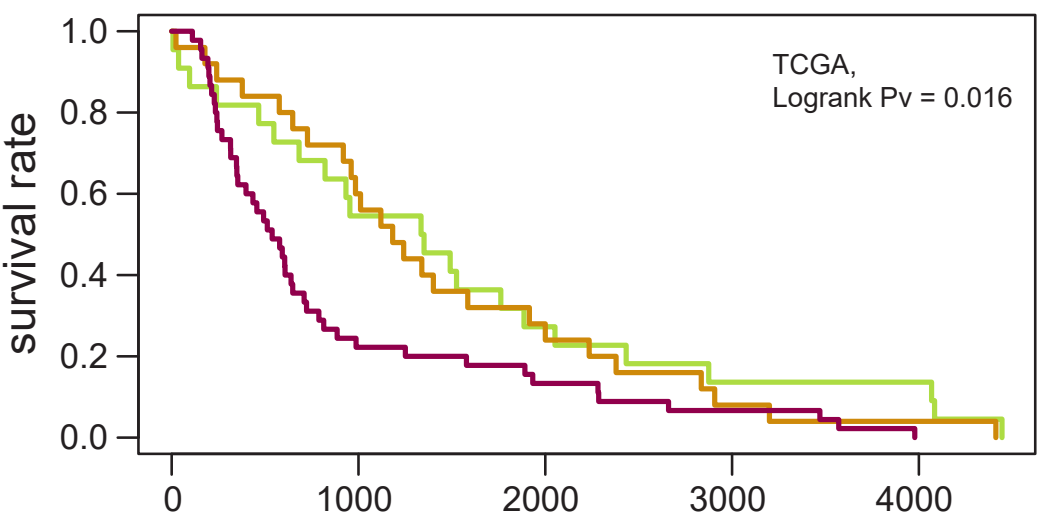

days

low

moderate

Patients stratified by

high

FOXM1 expression levels, only LGG samples

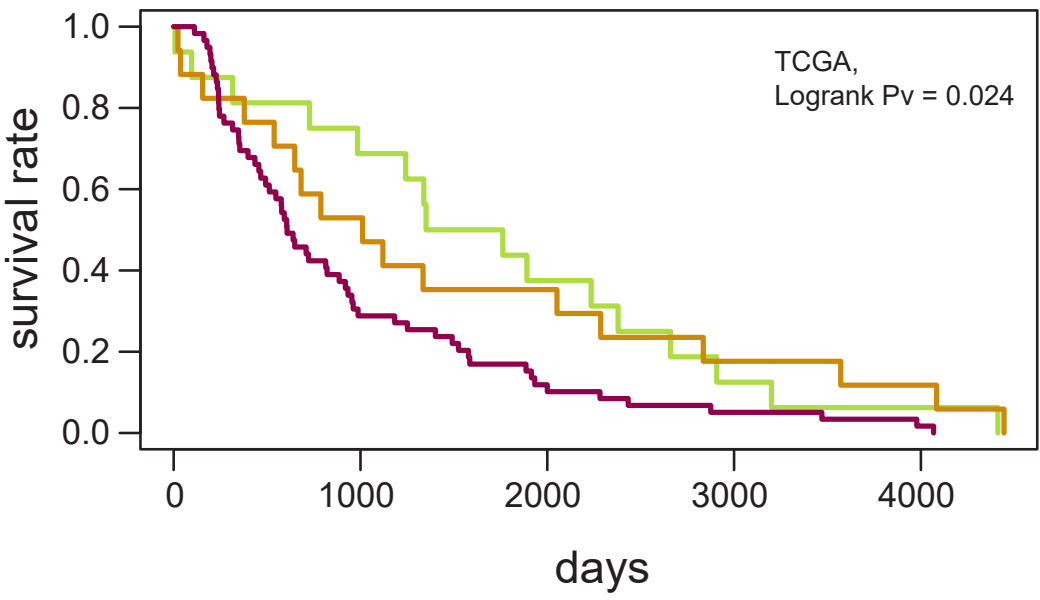




\section{Supplementary Figure 6}

A. Expression levels of genes coding for transcription factors identified to have a potential binding site in the putative ANXA2R enhancer region. Gene expression levels are represented as log-transformed FPKMs. The colour coding has been used to discriminate between major malignancy groups.

B. Kaplan-Meier curves plotted for the low grade glioma (LGG) data from TCGA database. The patients were stratified by either by ANXA2R (top part) or by FOXM1 (bottom part) expression levels into three equinumerous sub-groups. Differences in survival rates between sub-groups were assessed with the log-rank test. 